KA-TP-3-1998

hep-ph/9807427

\title{
Supersymmetric one-loop corrections to the process $e^{+} e^{-} \rightarrow f \bar{f}$
}

\author{
W. Hollik] and C. Schappacheri \\ Institut für Theoretische Physik \\ Universität Karlsruhe \\ Kaiserstraße 12 \\ D-76128 Karlsruhe, Germany
}

\begin{abstract}
Radiative one-loop corrections to fermion pair production in $e^{+} e^{-}$annihilation are calculated in the Minimal Supersymmetric Standard Model (MSSM). The size of the non-standard corrections is discussed for the process $e^{+} e^{-} \rightarrow \mu^{+} \mu^{-}$and $e^{+} e^{-} \rightarrow$ hadrons at LEP2 energies and for $e^{+} e^{-} \rightarrow t \bar{t}$ at a high energy $e^{+} e^{-}$collider. The relative difference between the predictions of the MSSM and the Standard Model is typically below $10 \%$.
\end{abstract}

*E-mail: hollik@particle.physik.uni-karlsruhe.de

${ }^{\dagger}$ E-mail: cs@particle.physik.uni-karlsruhe.de 


\section{Introduction}

In the light of electroweak precision experiments the Standard Model (SM) performance is almost perfect [1]. From a more theoretical point of view, however, supersymmetric versions of the SM seem more appropriate to overcome some of the conceptual problems of the SM, like the hierarchy problem or the non-occurrence of gauge coupling unification at high energies.

Tests of supersymmetric extensions of the SM hence are a central theme at present and future colliders. Supersymmetry (SUSY) [2] as an additional supporting symmetry is realized in its minimal version in terms of the Minimal Supersymmetric Standard Model (MSSM) [3, 4, 5]. Besides the possibility of direct production of SUSY particles at sufficiently high energies, the process of standard fermion pair production in $e^{+} e^{-}$annihilation offers the indirect search for virtual SUSY particles through quantum effects in terms of loop corrections.

In previous studies, complete 1-loop calculations have been performed for electroweak precision observables at the $Z$ resonance and for the $M_{W}-M_{Z}$ mass interdependence [6], with recent improvements by the 2-loop SUSY-QCD corrections to the $\rho$-parameter [7]. Investigation of $e^{+} e^{-} \rightarrow f \bar{f}$ above the $Z$ resonance, at LEP2 or a future linear collider, requires the extension of the previous calculations to the continuum region, which is the content of this paper. As a process of special interest, $t \bar{t}$ production is considered which will become experimentally accessible with high accuracy at a possible future $e^{+} e^{-}$collider.

In this paper we present a complete MSSM 1-loop calculation of the electroweak corrections and the non-standard part of the QCD corrections (SUSY-QCD corrections) to $e^{+} e^{-} \rightarrow f \bar{f}$ with $f=\mu, \tau, q$ and discuss the size of the possible virtual effects. Approximate results based on the SUSY 1-loop terms of $\mathcal{O}\left(\alpha m_{t}^{2} / M_{W}^{2}\right)$ have already been considered in [8], and SUSY-QCD corrections in [9]. For the decay of the top quark $t \rightarrow b W^{+}$, the MSSM 1-loop corrections are also available 10 .

This paper is organized as follows: Section 2 defines conventions and notations and gives the structure of the helicity amplitudes in terms of basic matrix elements, including the higher order terms. The results are discussed in Section 3 for the light fermion case and, with special emphasis, for top pair production. The appendix collects all the analytic expressions, required at the 1-loop level, in order to make the paper complete and self-contained. 


\section{The process $e^{+} e^{-} \rightarrow f \bar{f}$}

\subsection{Cross section and helicity amplitudes}

First we give the notations for the process $e^{+} e^{-} \rightarrow f \bar{f}(f=\mu, \tau, q)$. The momenta and helicities of the incoming electron and positron are denoted by $p, \kappa$ and $\bar{p}, \bar{\kappa}$, respectively. Correspondingly, $k, \eta$ and $\bar{k}, \bar{\eta}$ are used for the outgoing fermion $f$ and its antiparticle $\bar{f}$. The signs ' + ' and '-' of the variables $\kappa, \bar{\kappa}$ and $\eta, \bar{\eta}$ refer to helicities $+\frac{1}{2}$ and $-\frac{1}{2}$, respectively. The Mandelstam variables are defined by

$$
\begin{aligned}
s & =(p+\bar{p})^{2}=(k+\bar{k})^{2} \\
t & =(p-k)^{2}=(\bar{p}-\bar{k})^{2}, \\
u & =(p-\bar{k})^{2}=(\bar{p}-k)^{2} .
\end{aligned}
$$

The mass of the electron is neglected whenever possible. Since the helicity amplitudes for $e^{+} e^{-} \rightarrow$ $f \bar{f}(f=\mu, \tau, q)$ vanish for $\bar{\kappa}=\kappa$ in the limit $m_{e} \rightarrow 0$, we can write for the non-vanishing amplitudes

$$
\mathcal{M}(\kappa, \bar{\kappa}=-\kappa, \eta, \bar{\eta} ; s, t)=\mathcal{M}(\kappa, \eta, \bar{\eta} ; s, t) .
$$

Summing over the final helicities but keeping $\kappa$ fixed, we obtain the differential cross section for polarized initial states as follows:

$$
\frac{d \sigma}{d \Omega}(\kappa, s, \theta)=\frac{1}{64 \pi^{2} s} \beta_{f} N_{C}^{f} \sum_{\eta, \bar{\eta}}|\mathcal{M}(\kappa, \eta, \bar{\eta} ; s, t)|^{2}
$$

with

$$
\beta_{f}=\sqrt{1-\frac{4 m_{f}^{2}}{s}} \quad ; \quad N_{C}^{f}=3(1) \text { for quarks (leptons). }
$$

The scattering angle $\theta$ between $e^{-}$and $f$ in the center-of-mass system is related to $t, u$ via

$$
t=m_{f}^{2}-\frac{s}{2}\left(1-\beta_{f} \cos \theta\right), \quad u=m_{f}^{2}-\frac{s}{2}\left(1+\beta_{f} \cos \theta\right) .
$$

The cross section for unpolarized $e^{ \pm}$in the initial state is given by

$$
\frac{d \sigma}{d \Omega}(s, t)=\frac{1}{4}\left[\frac{d \sigma}{d \Omega}(+; s, t)+\frac{d \sigma}{d \Omega}(-; s, t)\right] .
$$

Besides the integrated unpolarized cross section

$$
\sigma=\int d \Omega \frac{d \sigma}{d \Omega}(s, t)
$$

it is of interest to consider the forward-backward asymmetry

$$
A_{F B}=\frac{\sigma^{F}-\sigma^{B}}{\sigma^{F}+\sigma^{B}}, \quad \sigma^{F(B)}=\int_{\theta<\frac{\pi}{2}\left(\theta>\frac{\pi}{2}\right)} d \Omega \frac{d \sigma}{d \Omega}(s, t)
$$

and the left-right asymmetry for polarized beams

$$
A_{L R}=\frac{\sigma_{L}-\sigma_{R}}{\sigma_{L}+\sigma_{R}}, \quad \sigma_{R, L}=\int d \Omega \frac{d \sigma}{d \Omega}( \pm ; s, t) .
$$


For practical calculations at the 1-loop level, it is convenient to decompose the helicity amplitude $\mathcal{M}$ into a set of basic matrix elements $\mathcal{M}_{i}^{\kappa \lambda}$ and corresponding invariant functions $L_{i}^{\kappa \lambda}$ according to

$$
\mathcal{M}(\kappa, \eta, \bar{\eta} ; s, t)=\sum_{i, \lambda} \mathcal{M}_{i}^{\kappa \lambda} L_{i}^{\kappa \lambda}
$$

with $(\bar{\kappa}=-\kappa)$ :

$$
\begin{aligned}
& \mathcal{M}_{1}^{\kappa \lambda}=\bar{v}(\bar{p}, \bar{\kappa}) \gamma_{\mu} \omega^{\kappa} u(p, \kappa) \bar{u}(k, \eta) \gamma^{\mu} \omega^{\lambda} v(\bar{k}, \bar{\eta}), \\
& \mathcal{M}_{2}^{\kappa \lambda}=\bar{v}(\bar{p}, \bar{\kappa})(\not k-\overline{\not k}) \omega^{\kappa} u(p, \kappa) \bar{u}(k, \eta)(\overline{\not p}-\not p) \omega^{\lambda} v(\bar{k}, \bar{\eta}), \\
& \mathcal{M}_{3}^{\kappa \lambda}=\bar{v}(\bar{p}, \bar{\kappa})(\not k-\not \bar{k}) \omega^{\kappa} u(p, \kappa) \bar{u}(k, \eta) \omega^{\lambda} v(\bar{k}, \bar{\eta}) \text {, } \\
& \mathcal{M}_{4}^{\kappa \lambda}=\bar{v}(\bar{p}, \bar{\kappa}) \gamma_{\mu} \omega^{\kappa} u(p, \kappa) \bar{u}(k, \eta)\left(\overline{\not p} \gamma^{\mu}+\gamma^{\mu} \not p\right) \omega^{\lambda} v(\bar{k}, \bar{\eta}), \\
& \mathcal{M}_{5}^{\kappa \lambda}=-\bar{v}(\bar{p}, \bar{\kappa}) \gamma_{\mu} \omega^{\lambda} v(\bar{k}, \bar{\eta}) \bar{u}(k, \eta) \gamma^{\mu} \omega^{\kappa} u(p, \kappa), \\
& \mathcal{M}_{6}^{\kappa \lambda}=-\bar{v}(\bar{p}, \bar{\kappa}) \not k \omega^{\lambda} v(\bar{k}, \bar{\eta}) \bar{u}(k, \eta) \overline{\not k} \omega^{\kappa} u(p, \kappa) \text {, } \\
& \mathcal{M}_{7}^{\kappa \lambda}=-\bar{v}(\bar{p}, \bar{\kappa}) \omega^{\lambda} v(\bar{k}, \bar{\eta}) \bar{u}(k, \eta) \omega^{\kappa} u(p, \kappa), \\
& \mathcal{M}_{8}^{\kappa \lambda}=-\bar{v}(\bar{p}, \bar{\kappa}) \not k \omega^{\lambda} v(\bar{k}, \bar{\eta}) \bar{u}(k, \eta) \omega^{\kappa} u(p, \kappa), \\
& \mathcal{M}_{9}^{\kappa \lambda}=-\bar{v}(\bar{p}, \bar{\kappa}) \omega^{\lambda} v(\bar{k}, \bar{\eta}) \bar{u}(k, \eta) \overline{\not k} \omega^{\kappa} u(p, \kappa), \\
& \mathcal{M}_{10}^{\kappa \lambda}=\bar{v}(\bar{p}, \bar{\kappa}) \gamma_{\mu} \omega^{\lambda} v(k, \eta) \bar{u}(\bar{k}, \bar{\eta}) \gamma^{\mu} \omega^{\kappa} u(p, \kappa), \\
& \mathcal{M}_{11}^{\kappa \lambda}=\bar{v}(\bar{p}, \bar{\kappa}) \overline{\not k} \omega^{\lambda} v(k, \eta) \bar{u}(\bar{k}, \bar{\eta}) \not k \omega^{\kappa} u(p, \kappa) \text {, } \\
& \mathcal{M}_{12}^{\kappa \lambda}=\bar{v}(\bar{p}, \bar{\kappa}) \omega^{\lambda} v(k, \eta) \bar{u}(\bar{k}, \bar{\eta}) \omega^{\kappa} u(p, \kappa), \\
& \mathcal{M}_{13}^{\kappa \lambda}=\bar{v}(\bar{p}, \bar{\kappa}) \overline{\not k} \omega^{\lambda} v(k, \eta) \bar{u}(\bar{k}, \bar{\eta}) \omega^{\kappa} u(p, \kappa), \\
& \mathcal{M}_{14}^{\kappa \lambda}=\bar{v}(\bar{p}, \bar{\kappa}) \omega^{\lambda} v(k, \eta) \bar{u}(\bar{k}, \bar{\eta}) \not k \omega^{\kappa} u(p, \kappa) \text {. }
\end{aligned}
$$

The chirality projectors $\omega^{ \pm}$are defined by

$$
\omega^{ \pm}=\frac{1}{2}\left(1 \pm \gamma_{5}\right)
$$

In the Born approximation only the two diagrams of Fig. 2.1 are relevant since the electronHiggs coupling is negligible. Therefore only (2.11a) contributes to the lowest-order amplitude $\mathcal{M}^{(0)}$ :

$$
\mathcal{M}^{(0)}(\kappa, \eta, \bar{\eta} ; s, t)=\sum_{\lambda} \mathcal{M}_{1}^{\kappa \lambda} L_{1}^{(0) \kappa \lambda}
$$

with

$$
L_{1}^{(0) \kappa \lambda}=i e^{2}\left(\frac{Q_{e} Q_{f}}{s}+\frac{g_{e}^{\kappa} g_{f}^{\lambda}}{s-M_{Z}^{2}}\right),
$$

$Q_{e}\left(Q_{f}\right)$ denotes the charge of the electron (fermion) and the couplings $g_{e}^{\kappa}$ and $g_{f}^{\lambda}$ can be found in the last line of Table 2.1.

Denoting the 1-loop contribution to the helicity amplitude by

$$
\mathcal{M}^{(1)}(\kappa, \eta, \bar{\eta} ; s, t)=\sum_{i, \lambda} \mathcal{M}_{i}^{\kappa \lambda} L_{i}^{(1) \kappa \lambda}
$$




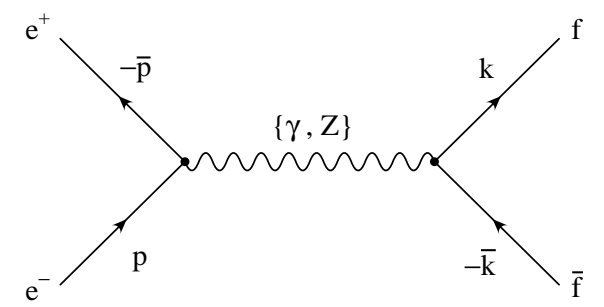

Figure 2.1: Born diagrams for the process $e^{+} e^{-} \rightarrow f \bar{f}$.

the differential cross section including $\mathcal{O}(\alpha)$ corrections is given by

$$
\begin{aligned}
\frac{d \sigma}{d \Omega}(\kappa, s, \theta)= & \frac{1}{64 \pi^{2} s} \beta_{f} N_{C}^{f} \sum_{\eta, \bar{\eta}}\left\{|\mathcal{M}(\kappa, \eta, \bar{\eta} ; s, t)|^{2}\right. \\
& \left.+2 \Re \mathrm{e}\left[\mathcal{M}^{(1)}(\kappa, \eta, \bar{\eta} ; s, t) \mathcal{M}^{(0) *}(\kappa, \eta, \bar{\eta} ; s, t)\right]\right\} .
\end{aligned}
$$

Since the invariant functions $L_{i}^{\kappa \lambda}$ are independent of the polarizations of the final-state fermions, the summation over these polarizations can be carried out directly for the products of the basic matrix elements:

$$
\begin{aligned}
& \sum_{\eta, \bar{\eta}} \mathcal{M}_{1}^{\kappa \lambda}\left(\mathcal{M}_{1}^{\kappa \lambda^{\prime}}\right)^{*}=4\left\{\delta_{\lambda^{\prime}}^{\lambda}\left[\delta_{\kappa}^{\lambda}\left(u-m_{f}^{2}\right)^{2}+\delta_{-\kappa}^{\lambda}\left(t-m_{f}^{2}\right)^{2}\right]+\delta_{-\lambda^{\prime}}^{\lambda} m_{f}^{2} s\right\}, \\
& \sum_{\eta, \bar{\eta}} \mathcal{M}_{2}^{\kappa \lambda}\left(\mathcal{M}_{1}^{\kappa \lambda^{\prime}}\right)^{*}=8\left(u t-m_{f}^{4}\right) \delta_{\lambda^{\prime}}^{\lambda}\left[\delta_{\kappa}^{\lambda}\left(u-m_{f}^{2}\right)-\delta_{-\kappa}^{\lambda}\left(t-m_{f}^{2}\right)\right] \\
& \sum_{\eta, \bar{\eta}} \mathcal{M}_{3}^{\kappa \lambda}\left(\mathcal{M}_{1}^{\kappa \lambda^{\prime}}\right)^{*}=-4 m_{f}\left(u t-m_{f}^{4}\right) \\
& \sum_{\eta, \bar{\eta}} \mathcal{M}_{4}^{\kappa \lambda}\left(\mathcal{M}_{1}^{\kappa \lambda^{\prime}}\right)^{*}=4 m_{f} s\left(\delta_{\lambda^{\prime}}^{\lambda}-\delta_{-\lambda^{\prime}}^{\lambda}\right)\left[\delta_{\kappa}^{\lambda^{\prime}}\left(u-m_{f}^{2}\right)-\delta_{-\kappa}^{\lambda^{\prime}}\left(t-m_{f}^{2}\right)\right] \\
& \sum_{\eta, \bar{\eta}} \mathcal{M}_{5}^{\kappa \lambda}\left(\mathcal{M}_{1}^{\kappa \lambda^{\prime}}\right)^{*}=4 \delta_{\lambda}^{\kappa}\left[\delta_{\lambda}^{\lambda^{\prime}}\left(u-m_{f}^{2}\right)^{2}+\delta_{-\lambda}^{\lambda^{\prime}} s m_{f}^{2}\right] \\
& \sum_{\eta, \bar{\eta}} \mathcal{M}_{6}^{\kappa \lambda}\left(\mathcal{M}_{1}^{\kappa \lambda^{\prime}}\right)^{*}=2 \delta_{\lambda}^{\kappa}\left\{\delta _ { \lambda } ^ { \lambda ^ { \prime } } \left[\left(u-m_{f}^{2}\right)^{2}\left(s-2 m_{f}^{2}\right)-2 m_{f}^{2}\left(u-m_{f}^{2}\right)\left(t-m_{f}^{2}\right)\right.\right. \\
& \left.\left.+s m_{f}^{4}\right]+\delta_{-\lambda}^{\lambda^{\prime}} m_{f}^{2}\left(u-m_{f}^{2}\right)^{2}\right\} \\
& \sum_{\eta, \bar{\eta}} \mathcal{M}_{7}^{\kappa \lambda}\left(\mathcal{M}_{1}^{\kappa \lambda^{\prime}}\right)^{*}=-2 \delta_{-\lambda}^{\kappa}\left[\delta_{\lambda}^{\lambda^{\prime}}\left(m_{f}^{2}-t\right)^{2}+\delta_{-\lambda}^{\lambda^{\prime}} s m_{f}^{2}\right] \\
& \sum_{\eta, \bar{\eta}} \mathcal{M}_{8}^{\kappa \lambda}\left(\mathcal{M}_{1}^{\kappa \lambda^{\prime}}\right)^{*}=m_{f} \delta_{\lambda}^{\kappa}\left\{\delta_{\lambda}^{\lambda^{\prime}}\left[\left(u-m_{f}^{2}\right)^{2}+s\left(s-2 m_{f}^{2}\right)-\left(t-m_{f}^{2}\right)^{2}\right]\right. \\
& \left.+\delta_{-\lambda}^{\lambda^{\prime}} 2\left(m_{f}^{2}-t\right)\left(m_{f}^{2}-u\right)\right\} \\
& \sum_{\eta, \bar{\eta}} \mathcal{M}_{9}^{\kappa \lambda}\left(\mathcal{M}_{1}^{\kappa \lambda^{\prime}}\right)^{*}=-m_{f} \delta_{-\lambda}^{\kappa}\left\{\delta_{\lambda}^{\lambda^{\prime}} 2\left(m_{f}^{2}-t\right)\left(m_{f}^{2}-u\right)\right.
\end{aligned}
$$




$$
\begin{aligned}
& \left.+\delta_{-\lambda}^{\lambda^{\prime}}\left[s\left(s-2 m_{f}^{2}\right)+\left(u-m_{f}^{2}\right)^{2}-\left(t-m_{f}^{2}\right)^{2}\right]\right\} \\
\sum_{\eta, \bar{\eta}} \mathcal{M}_{10}^{\kappa \lambda}\left(\mathcal{M}_{1}^{\kappa \lambda^{\prime}}\right)^{*}= & -4 \delta_{\lambda}^{\kappa}\left[\delta_{\lambda}^{-\lambda^{\prime}}\left(t-m_{f}^{2}\right)^{2}+\delta_{\lambda}^{\lambda^{\prime}} s m_{f}^{2}\right] \\
\sum_{\eta, \bar{\eta}} \mathcal{M}_{11}^{\kappa \lambda}\left(\mathcal{M}_{1}^{\kappa \lambda^{\prime}}\right)^{*}= & -2 \delta_{\lambda}^{\kappa}\left\{\delta _ { \lambda } ^ { - \lambda ^ { \prime } } \left[\left(t-m_{f}^{2}\right)^{2}\left(s-2 m_{f}^{2}\right)-2 m_{f}^{2}\left(t-m_{f}^{2}\right)\left(u-m_{f}^{2}\right)\right.\right. \\
& \left.\left.+s m_{f}^{4}\right]+\delta_{\lambda}^{\lambda^{\prime}} m_{f}^{2}\left(t-m_{f}^{2}\right)^{2}\right\} \\
\sum_{\eta, \bar{\eta}} \mathcal{M}_{12}^{\kappa \lambda}\left(\mathcal{M}_{1}^{\kappa \lambda^{\prime}}\right)^{*}= & 2 \delta_{-\lambda}^{\kappa}\left[\delta_{\lambda}^{-\lambda^{\prime}}\left(m_{f}^{2}-u\right)^{2}+\delta_{\lambda}^{\lambda^{\prime}} s m_{f}^{2}\right] \\
\sum_{\eta, \bar{\eta}} \mathcal{M}_{13}^{\kappa \lambda}\left(\mathcal{M}_{1}^{\kappa \lambda^{\prime}}\right)^{*}= & -m_{f} \delta_{\lambda}^{\kappa}\left\{\delta_{\lambda}^{-\lambda^{\prime}}\left[\left(t-m_{f}^{2}\right)^{2}+s\left(s-2 m_{f}^{2}\right)-\left(u-m_{f}^{2}\right)^{2}\right]\right. \\
& \left.+\delta_{\lambda}^{\lambda^{\prime}} 2\left(m_{f}^{2}-t\right)\left(m_{f}^{2}-u\right)\right\} \\
\sum_{\eta, \bar{\eta}} \mathcal{M}_{14}^{\kappa \lambda}\left(\mathcal{M}_{1}^{\kappa \lambda^{\prime}}\right)^{*}= & m_{f} \delta_{-\lambda}^{\kappa}\left\{\delta_{\lambda}^{-\lambda^{\prime}} 2\left(m_{f}^{2}-t\right)\left(m_{f}^{2}-u\right)\right. \\
& \left.+\delta_{\lambda}^{\lambda^{\prime}}\left[s\left(s-2 m_{f}^{2}\right)+\left(t-m_{f}^{2}\right)^{2}-\left(u-m_{f}^{2}\right)^{2}\right]\right\}
\end{aligned}
$$

\subsection{One-loop corrections}

The 1-loop contribution $\mathcal{M}^{(1)}(2.15)$ to the helicity amplitude contains the $\gamma$ and $Z$ self-energies, the $\gamma$ and $Z$ vertex corrections together with the external wave function renormalization, and the box diagrams:

$$
\mathcal{M}^{(1)}=\mathcal{M}_{S}+\mathcal{M}_{e V}+\mathcal{M}_{f V}+\mathcal{M}_{B}
$$

The complete set of vertex corrections comprises the QED corrections with virtual photons and the QCD corrections with virtual gluons for quark final states. They need real photon and gluon bremsstrahlung for a infrared finite result. The gauge invariant subclasses of "standard QED" and "standard QCD" corrections are identical to those in the Standard Model and are available in the literature [11, 12]. We therefore concentrate our discussion on the residual set of model dependent and IR-finite virtual corrections, which correspond to 2.18) without the diagrams involving virtual photons and gluons.

The supersymmetric part of the QCD corrections, arising from virtual gluinos, is included in (2.18) as part of the final state vertex correction $\mathcal{M}_{f V}$.

The concrete calculations are performed in the 't Hooft-Feynman gauge. Since the lowest order amplitudes contain only standard particles, we can apply the ON-SHELL renormalization scheme for the Standard Model [13] also for the MSSM case at 1-loop. The formal relations between 1-loop vertex functions and counter terms can be literally taken from the Standard Model; their explicit evaluation, however, requires the inclusion of the supersymmetric particles and the replacement of the standard Higgs contributions by those of the MSSM two Higgs doublets. 


\subsubsection{Vector boson propagator corrections}

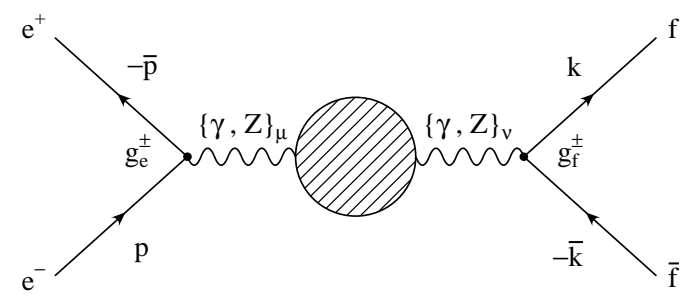

Figure 2.2: The self-energy corrections to $e^{+} e^{-} \rightarrow f \bar{f}$.

The 1-loop propagator correction appears in the sum (2.18) as the term (Fig. 2.2)

$$
\mathcal{M}_{S}=-i e^{2} \sum_{\lambda} \sum_{a, b=\gamma, Z} \frac{\hat{\Sigma}^{a b}(s)}{\left(s-M_{a}^{2}\right)\left(s-M_{b}^{2}\right)} g_{e}^{\kappa} g_{f}^{\lambda} \mathcal{M}_{1}^{\kappa \lambda}
$$

with $\mathcal{M}_{1}^{\kappa \lambda}$ from (2.11a), $M_{\gamma}=0$, and the renormalized self-energies $\hat{\Sigma}^{a b}(s)$ of the neutral vector bosons:

$$
\begin{aligned}
\hat{\Sigma}^{\gamma \gamma}(s)= & \Sigma^{\gamma \gamma}(s)-s \Pi^{\gamma \gamma}(0),\left.\quad \Pi^{\gamma \gamma}(0) \equiv \frac{\partial \Sigma^{\gamma \gamma}\left(q^{2}\right)}{\partial q^{2}}\right|_{q^{2}=0}, \\
\hat{\Sigma}^{Z Z}(s)= & \Sigma^{Z Z}(s)-\Re \mathrm{e} \Sigma^{Z Z}\left(M_{Z}^{2}\right)+\left(s-M_{Z}^{2}\right)\left\{-\Pi^{\gamma \gamma}(0)-2 \frac{c_{w}^{2}-s_{w}^{2}}{s_{w} c_{w}} \frac{\Sigma^{\gamma Z}(0)}{M_{Z}^{2}}\right. \\
& \left.+\frac{c_{w}^{2}-s_{w}^{2}}{s_{w}^{2}} \Re \mathrm{e}\left[\frac{\Sigma^{Z Z}\left(M_{Z}^{2}\right)}{M_{Z}^{2}}-\frac{\Sigma^{W W}\left(M_{W}^{2}\right)}{M_{W}^{2}}\right]\right\}, \\
\hat{\Sigma}^{\gamma Z}(s)= & \Sigma^{\gamma Z}(s)+\left(\frac{2 s}{M_{Z}^{2}}-1\right) \Sigma^{\gamma Z}(0)-s \frac{c_{w}}{s_{w}} \Re \mathrm{e}\left[\frac{\Sigma^{Z Z}\left(M_{Z}^{2}\right)}{M_{Z}^{2}}-\frac{\Sigma^{W W}\left(M_{W}^{2}\right)}{M_{W}^{2}}\right] .
\end{aligned}
$$

In the ON-SHELL scheme one has

$$
c_{w} \equiv \cos \theta_{w}=\frac{M_{W}}{M_{Z}}, \quad s_{w} \equiv \sin \theta_{w},
$$

and the couplings in (2.19) and Fig. 2.2 are listed in Table 2.1 with the following abbreviations:

$$
g_{Z, R}^{f(e)}=-\frac{s_{w}}{c_{w}} Q_{f(e)}, \quad g_{Z, L}^{f(e)}=\frac{I_{3}^{f(e)}-s_{w}^{2} Q_{f(e)}}{s_{w} c_{w}} .
$$

The quantities $\Sigma^{a b}\left(q^{2}\right)(a, b=\gamma, Z, W)$ denote the unrenormalized self-energies as the transverse coefficients in the expansion

$$
\Sigma_{\mu \nu}^{a b}(q)=-g_{\mu \nu} \Sigma^{a b}\left(q^{2}\right)+\frac{q_{\mu} q_{\nu}}{q^{2}}\left[\Sigma^{a b}\left(q^{2}\right)-\Sigma_{L}^{a b}\left(q^{2}\right)\right] .
$$

They are explicitly given in Appendix B.1. The $q_{\mu} q_{\nu}$-terms yield only contributions $\propto m_{e}^{2}$ in the ON-SHELL amplitudes and hence vanish in the limit $m_{e} \rightarrow 0$.

Since the energy domain above the $Z$ resonance is under consideration in this paper, the subtleties of the higher order contributions to (2.19), which are important for $Z$ physics (see, e.g., Ref. [14]), can be omitted in view of the experimental accuracy. Therefore we can restrict ourselves to the simplified $\mathcal{O}(\alpha)$ treatment according to (2.19), but we include the leading log resummation of the terms involving the light fermions. 


\begin{tabular}{|c|c||c|c|c|c|}
\hline$a$ & $b$ & $g_{e}^{+}$ & $g_{e}^{-}$ & $g_{f}^{+}$ & $g_{f}^{-}$ \\
\hline \hline$\gamma$ & $\gamma$ & $-Q_{e}$ & $-Q_{e}$ & $-Q_{f}$ & $-Q_{f}$ \\
\hline$\gamma$ & $Z$ & $-Q_{e}$ & $-Q_{e}$ & $g_{Z, R}^{f}$ & $g_{Z, L}^{f}$ \\
\hline$Z$ & $\gamma$ & $g_{Z, R}^{e}$ & $g_{Z, L}^{e}$ & $-Q_{f}$ & $-Q_{f}$ \\
\hline$Z$ & $Z$ & $g_{Z, R}^{e}$ & $g_{Z, L}^{e}$ & $g_{Z, R}^{f}$ & $g_{Z, L}^{f}$ \\
\hline
\end{tabular}

Table 2.1: Couplings.

\subsubsection{The vertex corrections}

The renormalized initial and final state vertex corrections together with the external wave function renormalizations are summarized by the contributions $\mathcal{M}_{e V}$ and $\mathcal{M}_{f V}$ to $\mathcal{M}^{(1)}(\kappa, \eta, \bar{\eta} ; s, t)$ in (2.18) (see Figs. 2.3 and 2.4):

$$
\mathcal{M}_{e V}=i \alpha^{2} \sum_{\lambda}\left(-\frac{1}{s} Q_{f} A_{e \gamma}^{\kappa}+\frac{1}{s-M_{Z}^{2}} g_{f}^{\lambda} A_{e Z}^{\kappa}\right) \mathcal{M}_{1}^{\kappa \lambda}
$$

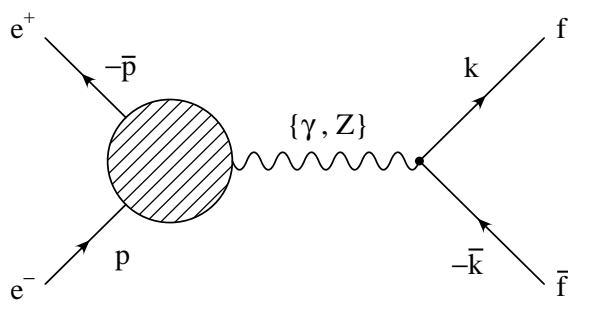

Figure 2.3: The electron vertex correction.

$$
\begin{aligned}
\mathcal{M}_{f V}=i \alpha^{2} \sum_{\lambda}\{ & \left(-\frac{1}{s} Q_{e} A_{f \gamma}^{\lambda}+\frac{1}{s-M_{Z}^{2}} g_{e}^{\kappa} A_{f Z}^{\lambda}\right) \mathcal{M}_{1}^{\kappa \lambda} \\
& \left.+\left(-\frac{1}{s} Q_{e} B_{f \gamma}^{\lambda}+\frac{1}{s-M_{Z}^{2}} g_{e}^{\kappa} B_{f Z}^{\lambda}\right) \mathcal{M}_{3}^{\kappa \lambda}\right\}
\end{aligned}
$$

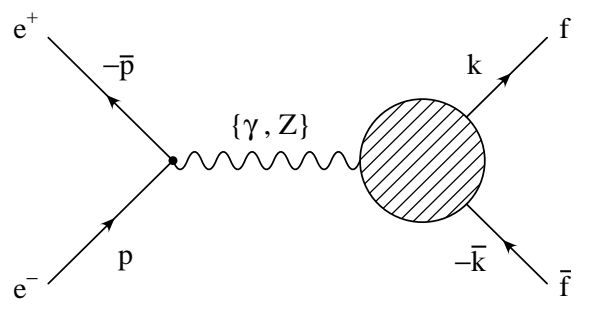

Figure 2.4: The vertex correction for outgoing fermions. 
The coefficients $A^{\lambda}$ and $B^{\lambda}$ are given by the following combinations $(a=\gamma, Z)$

$$
\begin{aligned}
A_{e a}^{\kappa} & =\left[A_{I}^{\kappa}+A_{I I}^{\kappa}+A_{V}^{\kappa}+A_{V I}^{\kappa}\right]_{e a}+\delta C_{e a}^{\kappa}, \\
A_{f a}^{\lambda} & =\left[A_{I}^{\lambda}+A_{I I}^{\lambda}+A_{I I I}^{\lambda}+A_{I V}^{\lambda}+A_{V}^{\lambda}+A_{V I}^{\lambda}\right]_{f a}+\delta C_{f a}^{\lambda}, \\
B_{f a}^{\lambda} & =\left[B_{I}^{\lambda}+B_{I I}^{\lambda}+B_{I I I}^{\lambda}+B_{I V}^{\lambda}+B_{V}^{\lambda}+B_{V I}^{\lambda}\right]_{f a} .
\end{aligned}
$$

The quantities in the brackets are listed in Appendix C. They are obtained as the form factors resulting from the various vertex classes $I, \ldots, V I$, summed over all individual contributions inside each class.

The counterterms for $F=e, f(f=\mu, \tau, q)$ read

$$
\begin{aligned}
\frac{\alpha}{4 \pi} \delta C_{F \gamma}^{-} & =\frac{I_{3}^{F}}{s_{w} c_{w}} \frac{\Sigma^{\gamma Z}(0)}{M_{Z}^{2}}+Q_{F} \delta Z_{F}^{(-)}, \\
\frac{\alpha}{4 \pi} \delta C_{F \gamma}^{+} & =Q_{F} \delta Z_{F}^{(+)} .
\end{aligned}
$$

In the case of the $Z$-fermion vertex the counterterms are given by

$$
\begin{aligned}
\frac{\alpha}{4 \pi} \delta C_{F Z}^{-} & =-\frac{I_{3}^{F} \Sigma^{\gamma Z}(0)}{s_{w}^{2} M_{Z}^{2}}-\frac{I_{3}^{F}-s_{w}^{2} Q_{F}}{s_{w} c_{w}} \delta Z_{F}^{(-)} \\
\frac{\alpha}{4 \pi} \delta C_{F Z}^{+} & =\frac{s_{w}}{c_{w}} Q_{F} \delta Z_{F}^{(+)}
\end{aligned}
$$

with the fermion field renormalization constants

$$
\delta Z_{F}^{( \pm)}=\Re \mathrm{e} \Sigma_{V}^{F}\left(m_{F}^{2}\right)+2 m_{F}^{2} \Re \mathrm{e}\left[\Sigma_{V}^{\prime F}\left(m_{F}^{2}\right)+\Sigma_{S}^{\prime F}\left(m_{F}^{2}\right)\right] \pm \Re \mathrm{e} \Sigma_{A}^{F}\left(m_{F}^{2}\right) .
$$

The fermion self-energies $\Sigma_{S, V, A}^{F}$ are listed explicitly in Appendix B.2.

\subsubsection{The box diagrams}

For the process $e^{+} e^{-} \rightarrow f \bar{f}(f=\mu, \tau, q)$ there are two different topologies of box diagrams, which we denote as 'direct' (Fig. D.1) and 'crossed' (Fig. D.2) box diagrams. The corresponding contribution to the 1-loop matrix element are labeled by $D$ and $C$, accordingly.

Since Higgs boson exchanges are negligible, we only have to consider the standard box graphs with $Z$ and $W^{ \pm}$exchange and the SUSY box graphs with neutralino and chargino exchange. As far as the charged current box diagrams are considered, for $I_{3}=-1 / 2$ only the direct box diagrams with $W^{ \pm}$and chargino exchange contribute, and for $I_{3}=+1 / 2$ only the crossed box diagrams with $W^{ \pm}$and chargino exchange.

The box contribution to the helicity amplitude (2.18) thus can be decomposed as follows:

$$
\mathcal{M}_{B}=\sum_{V=Z, W}\left(\mathcal{M}_{D}^{V}+\mathcal{M}_{C}^{V}\right)+\sum_{\tilde{\chi}=\tilde{\chi}^{0}, \tilde{\chi}^{+}}\left(\mathcal{M}_{D}^{\tilde{\chi}}+\mathcal{M}_{C}^{\tilde{\chi}}\right) .
$$

The summation has to be understood as extended over all neutralino and chargino configurations, and the corresponding $\tilde{e}_{1,2}, \tilde{\nu}_{e}$ and $\tilde{f}_{1,2}$ states. 
We use the following shorthand notations for combinations of 4-point tensor integral coefficients $D_{k l}$ and the scalar integrals $C_{0}$ and $D_{0}$ :

$$
\begin{aligned}
P_{1} & =4 i \alpha^{2} D_{27} \\
P_{2} & =2 i \alpha^{2}\left[2 C_{0}+\left\{\begin{array}{c}
t \\
u
\end{array}\right\}\left(D_{11}+D_{12}-D_{13}\right)-m_{f}^{2}\left(D_{11}-D_{12}+D_{13}\right)\right]\left\{\begin{array}{c}
\text { direct box } \\
\text { crossed box }
\end{array}\right. \\
P_{3} & =i \alpha^{2} D_{11} \\
P_{4} & =i \alpha^{2}\left(D_{11}-2 D_{13}\right) \\
P_{5} & =i \alpha^{2}\left(D_{11}-2 D_{12}\right) \\
P_{6} & =i \alpha^{2}\left(D_{11}+D_{24}-D_{25}\right) \\
P_{7} & =i \alpha^{2}\left[D_{11}+2\left(D_{23}-D_{13}-D_{26}\right)+D_{24}-D_{25}\right] \\
P_{8} & =i \alpha^{2}\left[D_{11}+2\left(D_{26}-D_{12}-D_{22}\right)+D_{24}-D_{25}\right] \\
P_{9} & =i \alpha^{2}\left(D_{25}-D_{26}\right) \\
P_{10} & =i \alpha^{2}\left(D_{24}-D_{26}\right) \\
P_{11} & =i \alpha^{2} D_{12} \\
P_{12} & =i \alpha^{2} D_{0} \\
P_{13} & =i \alpha^{2} D_{13} \\
P_{14} & =i \alpha^{2}\left(D_{25}-D_{23}\right) \\
P_{15} & =i \alpha^{2}\left(D_{24}-D_{22}\right)
\end{aligned}
$$

Further specifications are given in Appendix D.

With these abbreviations and with the matrix elements (2.11), the result for a direct vector boson box diagram is given by

$$
\begin{aligned}
\mathcal{M}_{D}^{V}= & \mathcal{M}_{1}^{\kappa \kappa}\left[P_{1} \lambda_{\kappa}^{+}-m_{f} m_{3}\left(P_{5} \bar{\lambda}_{\kappa}^{-}-P_{4} \bar{\lambda}_{\kappa}^{+}\right)\right]+\mathcal{M}_{1}^{\kappa-\kappa}\left[P_{2} \lambda_{\kappa}^{-}+m_{f} m_{3}\left(P_{5} \bar{\lambda}_{\kappa}^{+}-P_{4} \bar{\lambda}_{\kappa}^{-}\right)\right] \\
& +\mathcal{M}_{2}^{\kappa \kappa} P_{6} \lambda_{\kappa}^{+} \\
& +\mathcal{M}_{3}^{\kappa \kappa}\left[m_{f} P_{7} \lambda_{\kappa}^{+}-m_{3} P_{4} \bar{\lambda}_{\kappa}^{+}\right]-\mathcal{M}_{3}^{\kappa-\kappa}\left[m_{f} P_{8} \lambda_{\kappa}^{+}-m_{3} P_{5} \bar{\lambda}_{\kappa}^{-}\right] \\
& +\mathcal{M}_{4}^{\kappa \kappa} m_{3} P_{3} \bar{\lambda}_{\kappa}^{+}-\mathcal{M}_{4}^{\kappa-\kappa} m_{3} P_{3} \bar{\lambda}_{\kappa}^{-}
\end{aligned}
$$

and for a crossed vector boson box diagram by

$$
\begin{aligned}
\mathcal{M}_{C}^{V}= & \mathcal{M}_{1}^{\kappa \kappa}\left[-P_{2} \lambda_{\kappa}^{+}-m_{f} m_{3}\left(P_{5} \bar{\lambda}_{\kappa}^{-}-P_{4} \bar{\lambda}_{\kappa}^{+}\right)\right]+\mathcal{M}_{1}^{\kappa-\kappa}\left[-P_{1} \lambda_{\kappa}^{-}+m_{f} m_{3}\left(P_{5} \bar{\lambda}_{\kappa}^{+}-P_{4} \bar{\lambda}_{\kappa}^{-}\right)\right] \\
& +\mathcal{M}_{2}^{\kappa-\kappa} P_{6} \lambda_{\kappa}^{-} \\
& -\mathcal{M}_{3}^{\kappa \kappa}\left[m_{f} P_{7} \lambda_{\kappa}^{-}-m_{3} P_{4} \bar{\lambda}_{\kappa}^{-}\right]+\mathcal{M}_{3}^{\kappa-\kappa}\left[m_{f} P_{8} \lambda_{\kappa}^{-}-m_{3} P_{5} \bar{\lambda}_{\kappa}^{+}\right] \\
& -\mathcal{M}_{4}^{\kappa \kappa} m_{3} P_{3} \bar{\lambda}_{\kappa}^{-}+\mathcal{M}_{4}^{\kappa-\kappa} m_{3} P_{3} \bar{\lambda}_{\kappa}^{+} .
\end{aligned}
$$


$m_{3}$ can be read off from the Tables D.1 and D.2 in Appendix D.

The expression for a direct SUSY box diagram reads

$$
\begin{aligned}
\mathcal{M}_{D}^{\tilde{\chi}}= & \frac{1}{4} \mathcal{M}_{5}^{\kappa \kappa} P_{1} \bar{\eta}_{\kappa}^{+}-\mathcal{M}_{6}^{\kappa \kappa} P_{9} \bar{\eta}_{\kappa}^{+} \\
& +\mathcal{M}_{7}^{\kappa-\kappa}\left[m_{f}^{2} P_{10} \bar{\eta}_{\kappa}^{+}-m_{f} m_{2}\left(P_{3}-P_{13}\right) \eta_{\kappa}^{-}-m_{f} m_{4} P_{11} \eta_{\kappa}^{+}+m_{2} m_{4} P_{12} \bar{\eta}_{\kappa}^{-}\right] \\
& +\mathcal{M}_{8}^{\kappa \kappa}\left[m_{4} P_{13} \eta_{\kappa}^{+}-m_{f} P_{14} \bar{\eta}_{\kappa}^{+}\right] \\
& +\mathcal{M}_{9}^{\kappa-\kappa}\left[m_{f} P_{15} \bar{\eta}_{\kappa}^{+}-m_{2}\left(P_{3}-P_{11}\right) \eta_{\kappa}^{-}\right] .
\end{aligned}
$$

For a crossed SUSY box diagram we have

$$
\begin{aligned}
\mathcal{M}_{C}^{\tilde{\chi}}= & \frac{1}{4} \mathcal{M}_{10}^{\kappa \kappa} P_{1} \bar{\eta}_{\kappa}^{+}-\mathcal{M}_{11}^{\kappa \kappa} P_{9} \bar{\eta}_{\kappa}^{+} \\
& +\mathcal{M}_{12}^{\kappa-\kappa}\left[m_{f}^{2} P_{10} \bar{\eta}_{\kappa}^{+}-m_{f} m_{2}\left(P_{3}-P_{13}\right) \eta_{\kappa}^{-}-m_{f} m_{4} P_{11} \eta_{\kappa}^{+}+m_{2} m_{4} P_{12} \bar{\eta}_{\kappa}^{-}\right] \\
& +\mathcal{M}_{13}^{\kappa \kappa}\left[m_{4} P_{13} \eta_{\kappa}^{+}-m_{f} P_{14} \bar{\eta}_{\kappa}^{+}\right] \\
& +\mathcal{M}_{14}^{\kappa-\kappa}\left[m_{f} P_{15} \bar{\eta}_{\kappa}^{+}-m_{2}\left(P_{3}-P_{11}\right) \eta_{\kappa}^{-}\right] .
\end{aligned}
$$

$m_{2}, m_{4}$ denotes charginos or neutralinos, respectively, and can be read off from the Tables D.3, D.4, D.5 and D.6 in Appendix D.

The following abbreviations for products of the couplings $g_{1,2,3,4}^{\kappa}$ specified in Appendix D have been used in the expressions 2.32 - (2.35):

$$
\begin{array}{rlrl}
\lambda_{\kappa}^{ \pm} & =g_{1}^{\kappa} g_{2}^{\kappa} g_{3}^{ \pm \kappa} g_{4}^{ \pm \kappa}, & \bar{\lambda}_{\kappa}^{ \pm}=g_{1}^{\kappa} g_{2}^{\kappa} g_{3}^{ \pm \kappa} g_{4}^{\mp \kappa}, \\
\eta_{\kappa}^{ \pm}=g_{1}^{-\kappa} g_{2}^{\kappa} g_{3}^{ \pm \kappa} g_{4}^{ \pm \kappa}, & \bar{\eta}_{\kappa}^{ \pm}=g_{1}^{-\kappa} g_{2}^{\kappa} g_{3}^{ \pm \kappa} g_{4}^{\mp \kappa} .
\end{array}
$$




\section{Numerical analysis and discussion}

In this section we discuss the numerical effects of the weak and of the SUSY-QCD corrections in the reaction $e^{+} e^{-} \rightarrow f \bar{f}(f=\mu, \tau, q)$.

For cross-checking our results ?, numerical comparisons have been performed with the already available subset of the SM and 2-Higgs doublet contributions [16] and with the MSSM gauge boson self-energies as given in [17] by utilizing the computer codes of Ref. [16, 17]. The comparisons show perfect agreement in all cases.

In order to exhibit the deviations from the SM induced by supersymmetry, we introduce the quantity

$$
\Delta(s)=\frac{\sigma^{\operatorname{MSSM}}(s)-\sigma^{\mathrm{SM}}(s)}{\sigma^{(0)}(s)},
$$

for the unpolarized cross section, and the differences

$$
\begin{aligned}
\delta A_{F B}(s) & =A_{F B}^{\mathrm{MSSM}}(s)-A_{F B}^{\mathrm{SM}}(s), \\
\delta A_{L R}(s) & =A_{L R}^{\mathrm{MSSM}}(s)-A_{L R}^{\mathrm{SM}}(s),
\end{aligned}
$$

for the forward-backward and left-right asymmetries. $\sigma^{(0)}(s)$ is the total cross section in the Born approximation corresponding to Eqs. (2.13) and (2.14).

For the numerical analysis, we used the MSSM parameters as specified in Appendix A; they are chosen to be consistent with the experimental constraints [1, 18]. We further used the top quark mass of $m_{t}=175 \mathrm{GeV}$ in the whole paper. Note that without the SUSY-GUT relation (A.10) for $M_{3}$, the gluino mass $m_{\tilde{g}} \equiv\left|M_{3}\right|$ is another input parameter. In the case of mixed sfermions (see Appendix A) we chose for further simplicity $A_{u}=A_{d}=A$.

We split the discussion into two parts: the light fermion case, which is experimentally accessible at LEP, and the case of $t \bar{t}$ production.

\subsection{Results for $e^{+} e^{-} \rightarrow \mu^{+} \mu^{-}$}

In the absence of direct discoveries of SUSY particles at LEP, the question for indirect effects in the standard cross sections, resulting from virtual SUSY contributions, is of special interest.

In view of the claimed accuracy in the cross section measurement of $1.3 \%$ for $\mu^{+} \mu^{-}$and $0.7 \%$ for hadron final states [19], it is of basic importance to study the size of the MSSM 1loop contributions obtained from varying the model parameters in a wide range consistent with experimental and theoretical constraints. Since for heavy non-standard particles one recovers the Standard Model with a light Higgs boson $\left(m_{h^{0}} \lesssim 130 \mathrm{GeV}\right)$, the largest deviations will occur for light SUSY particles close to their detection limits.

In Fig. 3.1 we display the range for the quantity $\Delta$, Eq. (3.1), from scanning the SUSY parameters, varying $m_{\tilde{t}_{1}}$ between 100 and $500 \mathrm{GeV}, \mu$ between -500 and $500 \mathrm{GeV}$ (with the region $-100 \mathrm{GeV}<\mu<100 \mathrm{GeV}$ excluded) in steps of $50 \mathrm{GeV}$, and $M_{2}$ between 200 and 1000 $\mathrm{GeV}$ in steps of $10 \mathrm{GeV}$ in a low $\tan \beta$ scenariof $(\tan \beta=1.6)$. The deviations from the $\mathrm{SM}$ are always negative, and $\Delta$ can reach the maximum of $-1.7 \%$ at $M_{2}=1000 \mathrm{GeV}$ which is close to

\footnotetext{
${ }^{1}$ We used the program library AAFF $\sqrt{15}$ for the calculation of the tensor integrals.

${ }^{2}$ In addition, we chose steps of $10 \mathrm{GeV}$ (instead of $\left.50 \mathrm{GeV}\right)$ for the case $A=m_{\tilde{t}_{1}}$ without SUSY box diagrams (which is not depicted here) and the result differs less than $\pm 0.1 \%$ from both results in Fig. 3.1.
} 
the experimental precision. There is (almost) no difference between the choice of a fixed $A=m_{\tilde{t}_{1}}$ (which is not depicted here) and for $-400 \mathrm{GeV} \leq A \leq 400 \mathrm{GeV}$. The large negative values are due to the light sfermion masses which can occur for our set of parameters.

In the large $\tan \beta$ scenario $(\tan \beta=40)$ the predicted range for the same parameter variations is smaller than in Fig. $3.1(-1 \% \lesssim \Delta \lesssim 0 \%)$. This is essentially due to the fact that for large $\tan \beta$ the lightest allowed sfermion masses are in general larger than in the low $\tan \beta$ case. Since the largest values of $\Delta$ occur for the lightest possible sfermion masses this leads to the smaller result.

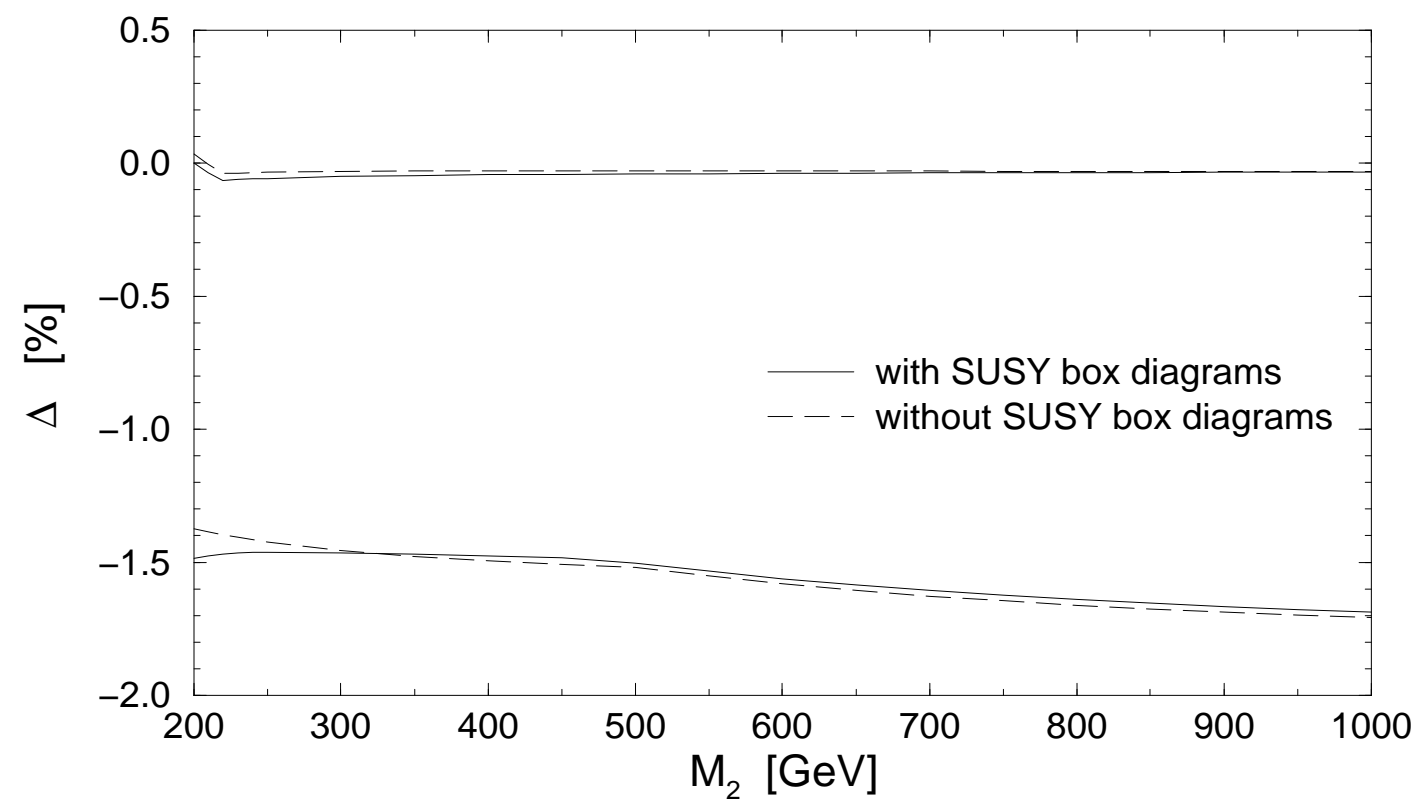

Figure 3.1: $e^{+} e^{-} \rightarrow \mu^{+} \mu^{-}$. Predicted range of $\Delta$ for $\sqrt{s}=192 \mathrm{GeV}, \tan \beta=1.6, M_{A}=500$ $\mathrm{GeV}, 100 \mathrm{GeV} \leq m_{\tilde{t}_{1}} \leq 500 \mathrm{GeV},-400 \mathrm{GeV} \leq A \leq 400 \mathrm{GeV}$ and $-500 \mathrm{GeV} \leq \mu \leq 500 \mathrm{GeV}$ (but $-100 \mathrm{GeV}<\mu<100 \mathrm{GeV}$ is excluded). The predicted range is between the two upper and the two lower lines.

\section{$3.2 \quad$ Results for $e^{+} e^{-} \rightarrow \sum_{q \neq t} q \bar{q}$}

At LEP energies, the hadron production process from primary quarks $\neq t$ is experimentally accessible, with a large cross section and a final accuracy of $0.7 \%$ [19].

In Fig. 3.2 we display the results for $\Delta$ from SUSY parameter scans, in which we vary $m_{\tilde{t}_{1}}$ between 100 and $500 \mathrm{GeV}, \mu$ between -500 and $500 \mathrm{GeV}$ (but $-100 \mathrm{GeV}<\mu<100 \mathrm{GeV}$ is excluded) in steps of $50 \mathrm{GeV}$, and $M_{2}$ between 200 and $1000 \mathrm{GeV}$, in steps of $10 \mathrm{GeV}$ in the low $\tan \beta$ scenario.

The deviations from the SM are always negative and $\Delta$ can reach up to $-3.7 \%$. The difference to the lepton case (Fig. 3.1) is essentially caused by the presence of the extra gluino exchange in the SUSY-QCD corrections. There is (almost) no difference between the choice of a fixed $A=m_{\tilde{t}_{1}}$ (which is not depicted here) or for $-400 \mathrm{GeV} \leq A \leq 400 \mathrm{GeV}$.

In the large $\tan \beta$ scenario the predicted range for the same parameter variations is smaller than in Fig. $3.2(-1.8 \% \lesssim \Delta \lesssim 0 \%)$, for the same reasons as in Section 3.1. 


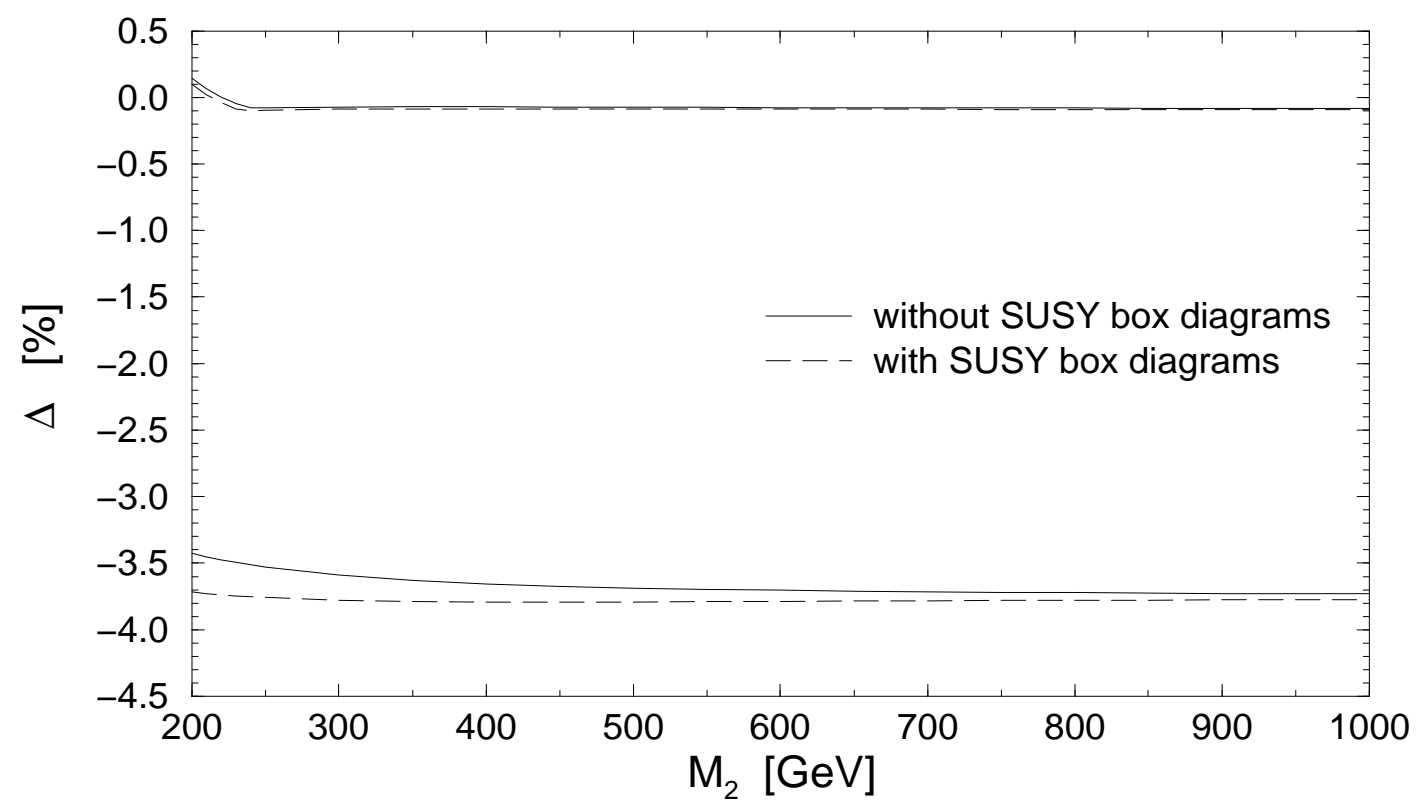

Figure 3.2: $e^{+} e^{-} \rightarrow \sum_{q \neq t} q \bar{q}$. Predicted range of $\Delta$ for $\sqrt{s}=192 \mathrm{GeV}, \tan \beta=1.6, M_{A}=500$ $\mathrm{GeV}, 100 \mathrm{GeV} \leq m_{\tilde{t}_{1}} \leq 500 \mathrm{GeV},-400 \mathrm{GeV} \leq A \leq 400 \mathrm{GeV}$ and $-500 \mathrm{GeV} \leq \mu \leq 500 \mathrm{GeV}$ (but $-100 \mathrm{GeV}<\mu<100 \mathrm{GeV}$ is excluded). The predicted range is between the two upper and the two lower lines.

\section{$3.3 \quad$ Results for $e^{+} e^{-} \rightarrow t \bar{t}$}

The production of $t \bar{t}$-pairs is a process of basic interest at a future high energy $e^{+} e^{-}$collider and will provide precision studies of the top quark properties (see, e.g., Ref. [20]). From the large top quark mass one expects a special sensitivity to the structure of the Yukawa couplings.

1-loop electroweak corrections for $t \bar{t}$ production have been calculated in the SM both for the threshold region [21] and the continuum [11, 22]. Also for Two-Higgs-Doublet Models (2HDM) the electroweak radiative corrections are available [16, 23]. When the SUSY constraints on the 2HDM Higgs sector are imposed, the potentially large Higgs-induced corrections are kept typically below $\sim 2 \%$ [16.

Within the MSSM the SUSY-QCD corrections to $t \bar{t}$ production have been calculated in [9], and the subclass of electroweak 1-loop contributions $\propto m_{t}^{2} / M_{W}^{2}$ resulting from the top Yukawa couplings has been discussed in [8]. Our results are in qualitative agreement with Ref. [8]. Quantitatively they differ by a few percent, which, however, is not surprising in view of the approximations in Ref. [8]. It is also known from the standard model case that the restriction to the terms $\propto m_{t}^{2} / M_{W}^{2}$ only yields a poor approximation of the complete result. Our discussion in this section is complete at the 1-loop level, including also the SUSY-QCD corrections.

In order to give an idea of the absolute size of the total cross section, we plot $\sigma^{(0)}(s)$ and at the 1-loop level $\sigma^{\mathrm{SM}}(s)$ and $\sigma^{\mathrm{MSSM}}(s)$ in Fig. 3.3 for a typical choice of parameters as a function of the center-of-mass energy. The strong deviation of the Born cross section, parameterized by $G_{\mu}$, from the 1-loop result, both for SM and MSSM, is essentially due to the box diagram contributions. In addition, Fig. 3.4 displays the Born cross section and the total cross section $\sigma^{\operatorname{MSSM}}(s)$ with and without box diagram contributions in dependence of the light top squark mass $m_{\tilde{t}_{1}}$, also 
for a typical set of parameters. This figure shows that the box diagram contributions to $\Delta$ can reach about $10 \%$ of the MSSM 1-loop result and are therefore non-negligible.

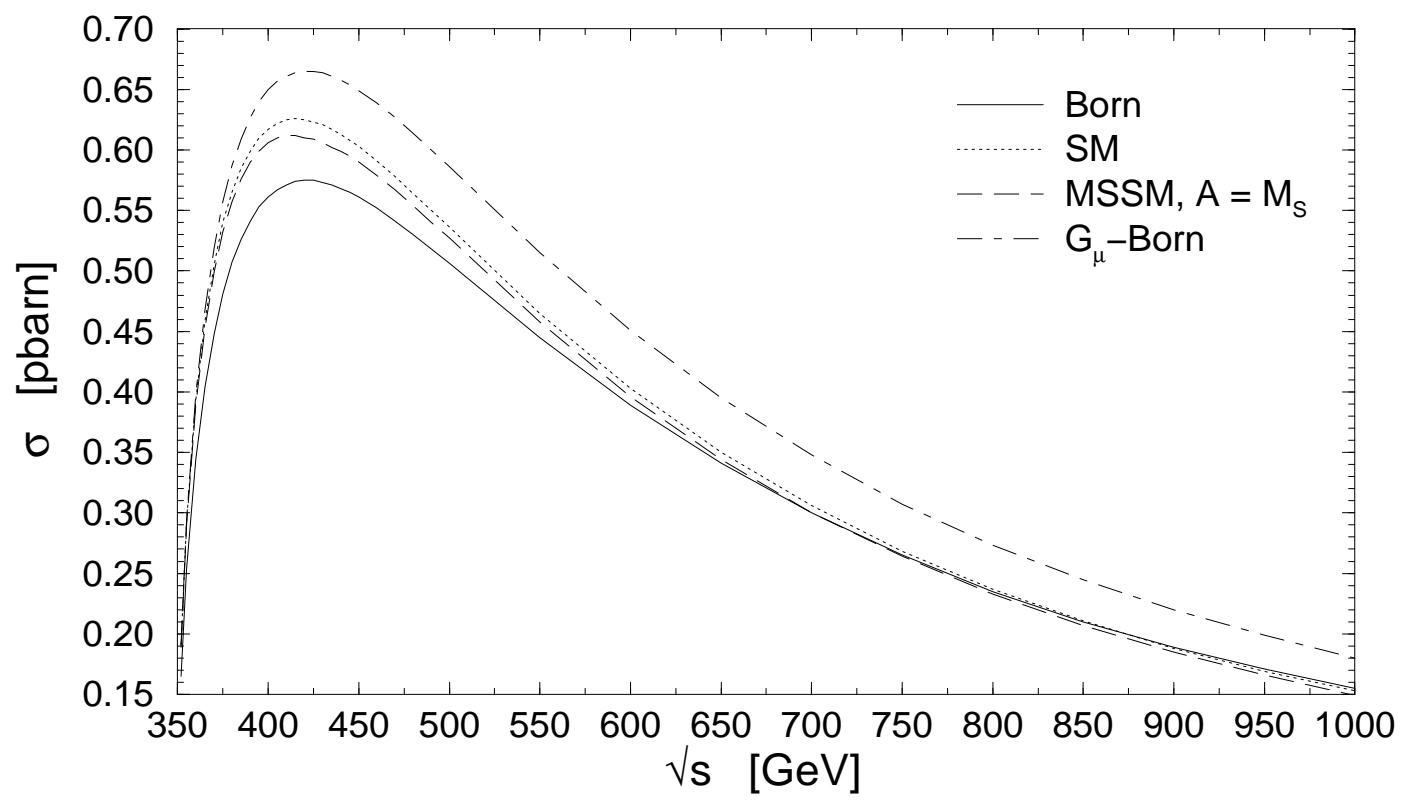

Figure 3.3: $e^{+} e^{-} \rightarrow t \bar{t}$. Energy dependence of $\sigma$ for $\tan \beta=40, M_{A}=200 \mathrm{GeV}, A=M_{S}=200$ $\mathrm{GeV}, \mu=-150 \mathrm{GeV}$ and $M_{2}=250 \mathrm{GeV}$.

The energy dependence of $\Delta$ is illustrated in Fig. 3.5 for low and high $\tan \beta$ with and without sfermion mixing for a fixed set of SUSY parameters. With increasing $s$, the values of $\Delta$ become negative in all cases.

The dependence of $\Delta$ on the common squark mass parameter $M_{S}$, Eq. (A.2), is shown in Fig. 3.6 at $\sqrt{s}=500 \mathrm{GeV}$. For large $M_{S}, \Delta$ is very small in accordance with the decoupling properties of heavy sfermions [24]. The steep behavior of the dotted curve for $\tan \beta=40$ at low $M_{S}$ is related to low chargino and sbottom masses, close to their present exclusion limits. It enters through the top wave function renormalization with a threshold singularity at $m_{t}=m_{\tilde{\chi}_{1}^{+}}+m_{\tilde{b}_{2}}$. Releasing the GUT constraint (A.10) for the gluino mass and treating $m_{\tilde{g}}$ as an independent parameter leads to a variation of the results in Fig. 3.6 within $\pm 1 \%$. The asymmetries $A_{L R}, A_{F B}$ (which are not depicted here) are not very sensitive to the SUSY contributions 5 : their variations are in the ranges $-9 \times 10^{-3}<\delta A_{L R}<2 \times 10^{-3}$ and $-7 \times 10^{-3}<\delta A_{F B}<1 \times 10^{-3}$ for the same parameters as in Fig. 3.6.

Fig. 3.7 illustrates the $\tan \beta$ dependence of $\Delta$ for various values of $M_{S}$ with and without sfermion mixing. Again, the singularity for high $\tan \beta$ is related to the threshold $m_{t}=m_{\tilde{\chi}_{1}^{+}}+m_{\tilde{b}_{2}}$ in the top wave function renormalization. For the parameters of Fig. 3.7 the contributions to the asymmetries cover the ranges $-9 \times 10^{-3}<\delta A_{L R}<5 \times 10^{-3}$ and $-6 \times 10^{-3}<\delta A_{F B}<1 \times 10^{-3}$.

The dependence of $\Delta$ on the gluino mass $m_{\tilde{g}}$ is shown in Fig. 3.8. Now $m_{\tilde{g}}$ is treated as an independent parameter. For the purpose of illustration we also keep the range of small gluino

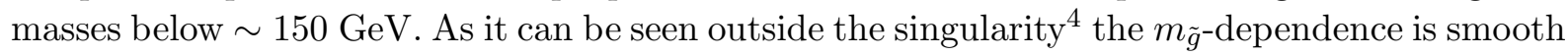

\footnotetext{
${ }^{3} A_{L R}$ and $A_{F B}$ are of the order $\mathcal{O}\left(5 \times 10^{-1}\right)$

${ }^{4}$ The singularity in Fig. 3.8 corresponds to the case $m_{t}=m_{\tilde{g}}+m_{\tilde{t}_{1}}$.
} 


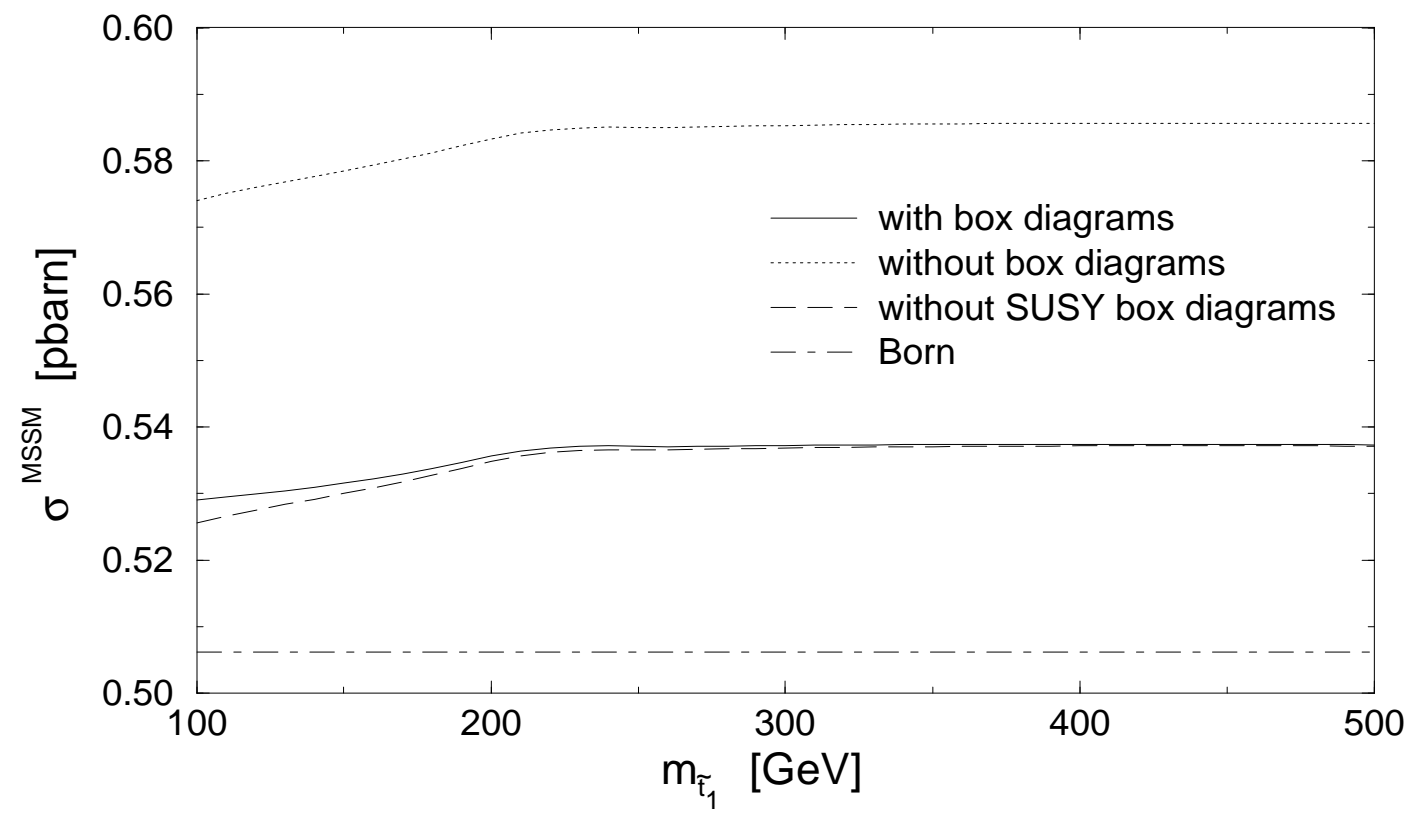

Figure 3.4: $e^{+} e^{-} \rightarrow t \bar{t}$. The $m_{\tilde{t}_{1}}$ dependence of $\sigma$ for $\sqrt{s}=500 \mathrm{GeV}, \tan \beta=1.6, M_{A}=200$ $\mathrm{GeV}, \mu=-150 \mathrm{GeV}, M_{2}=200 \mathrm{GeV}$ and $A=m_{\tilde{t}_{1}}$.

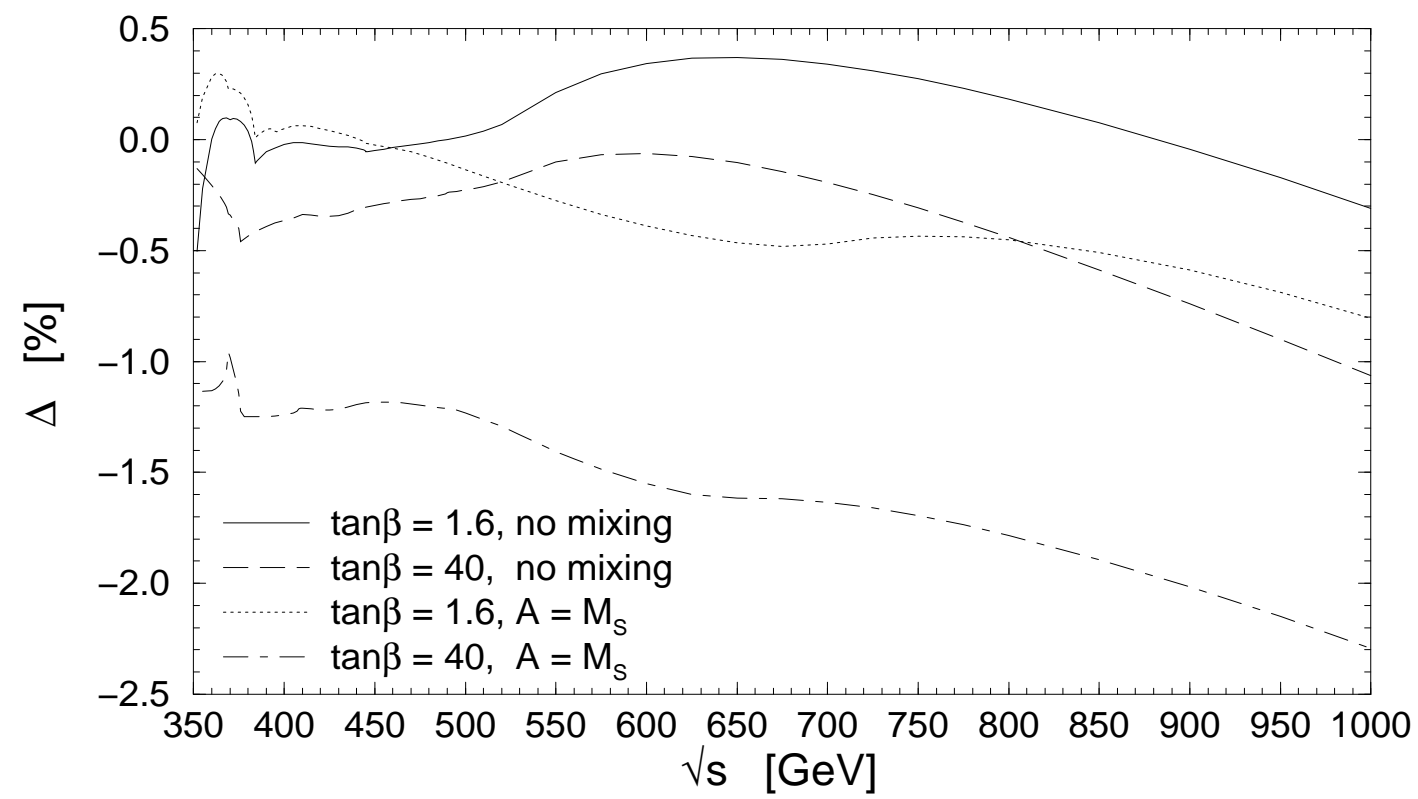

Figure 3.5: $e^{+} e^{-} \rightarrow t \bar{t}$. The $\sqrt{s}$ dependence of $\Delta$ for $M_{A}=200 \mathrm{GeV}, M_{S}=200 \mathrm{GeV}, \mu=-150$ $\mathrm{GeV}$ and $M_{2}=200 \mathrm{GeV}$. "no mixing" denotes unmixed sfermions. 


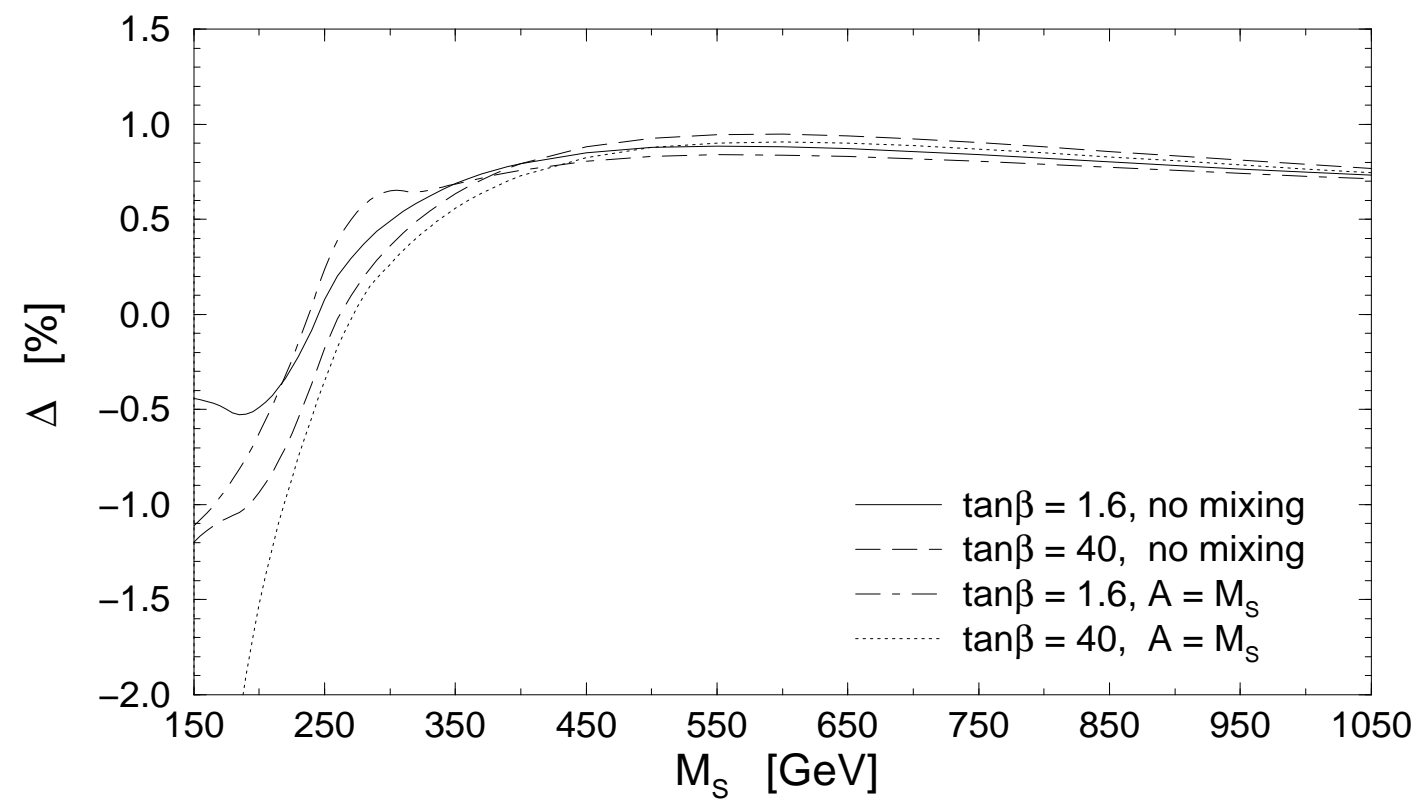

Figure 3.6: $e^{+} e^{-} \rightarrow t \bar{t}$. The $M_{S}$ dependence of $\Delta$ for $\sqrt{s}=500 \mathrm{GeV}, M_{A}=200 \mathrm{GeV}, \mu=-100$ $\mathrm{GeV}$ and $M_{2}=250 \mathrm{GeV}$. "no mixing" denotes unmixed sfermions.

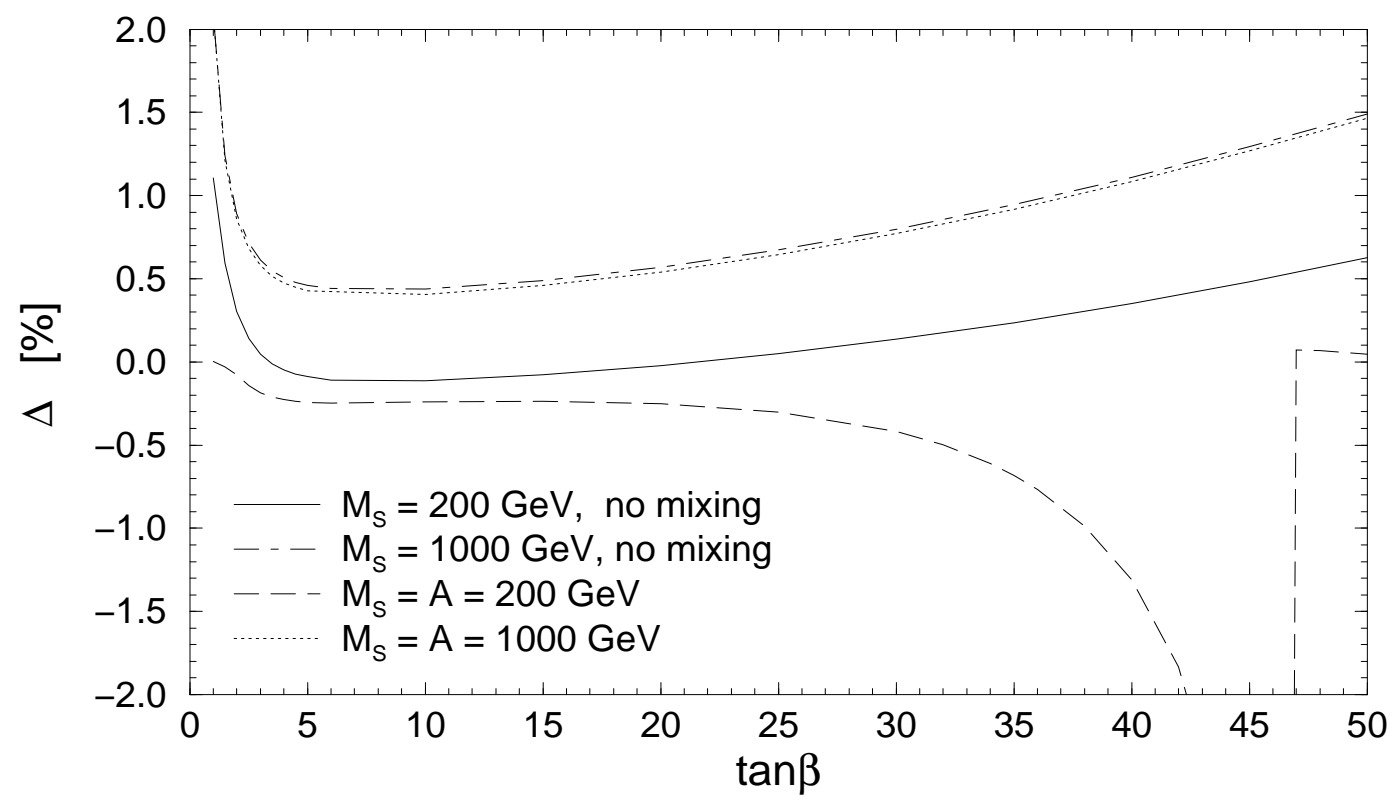

Figure 3.7: $e^{+} e^{-} \rightarrow t \bar{t}$. The $\tan \beta$ dependence of $\Delta$ for $\sqrt{s}=500 \mathrm{GeV}, M_{A}=200 \mathrm{GeV}$, $\mu=-150 \mathrm{GeV}$ and $M_{2}=100 \mathrm{GeV}$. "no mixing" denotes unmixed sfermions. 
and even very light gluinos do not give rise to significantly different predictions. For large $\tan \beta$ and for the specific constellation of masses $m_{\tilde{t}_{1}}=m_{t}$, corrections of the order $-6 \%$ are obtained. But this is due to the fact that for these specific parameters we are near a threshold singularity at $m_{t}=m_{\tilde{\chi}_{1}^{+}}+m_{\tilde{b}_{2}}$, which is an electroweak effect. Our results for the SUSY-QCD part of the 1-loop corrections are in agreement with the results of Ref. [9].

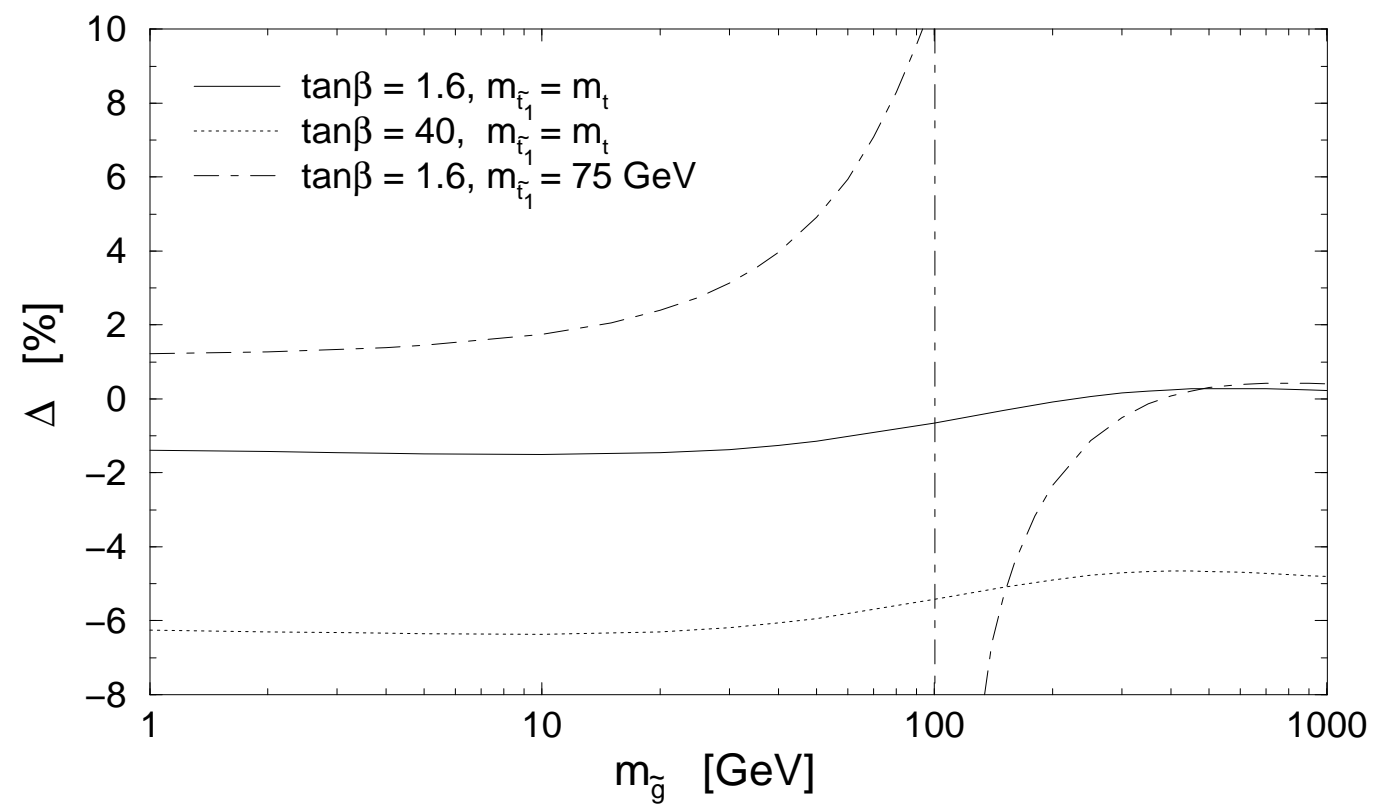

Figure 3.8: $e^{+} e^{-} \rightarrow t \bar{t}$. The $m_{\tilde{g}}$ dependence of $\Delta$ for $\sqrt{s}=500 \mathrm{GeV}, M_{A}=150 \mathrm{GeV}, \mu=-150$ $\mathrm{GeV}, A=m_{\tilde{t}_{1}}$ and $M_{2}=150 \mathrm{GeV}$.

For a more detailed discussion of the weak corrections, the dependence of $\Delta$ on the lighter chargino mass $m_{\tilde{\chi}_{1}^{+}}$is shown in Fig. 3.9. Again the singularities correspond to thresholds in the top wave function renormalization. Given the experimental restrictions on $m_{\tilde{\chi}_{1}^{+}}$and $m_{\tilde{t}_{1}}$, the values of $\Delta$ are typically within a few percent.

The result of a SUSY parameter scan for low $\tan \beta$ is presented in Fig. 3.10, where $A=m_{\tilde{t}_{1}}$ is varied between 100 and $500 \mathrm{GeV}, \mu$ between -500 and $500 \mathrm{GeV}$ (but $-100 \mathrm{GeV}<\mu<100 \mathrm{GeV}$ is excluded) in steps of $50 \mathrm{GeV}$, and $M_{2}$ between 200 and $1000 \mathrm{GeV}$ in steps of $10 \mathrm{GeV}$. The influence of the box diagrams is shown separately (solid line). The dip around $M_{2}=331 \mathrm{GeV}$ (solid and dashed line) is a threshold singularity at $m_{t}=m_{\tilde{\chi}_{1}^{+}}+m_{\tilde{b}_{1}}$. The dip around $M_{2}=$ $398 \mathrm{GeV}$ (dashed line) is a threshold effect corresponding to $m_{\tilde{\chi}_{1}^{+}}+m_{\tilde{\chi}_{2}^{+}}=\sqrt{s}$ from the crossed chargino box, because the $f \tilde{f}^{\prime} \tilde{\chi}^{+}$couplings are (for this parameters) up to 10 times larger then the $Z \tilde{\chi}_{1}^{+} \tilde{\chi}_{2}^{+}$coupling in the corresponding self-energy and vertex diagrams. (Therefore this threshold is invisible in the dashed line.) The large values are due to nearby singularity effects at $m_{t}=m_{\tilde{\chi}_{1}^{+}}+m_{\tilde{b}_{1,2}}(\Delta \sim-7 \%)$ and production threshold effects at $m_{\tilde{\chi}_{4}^{0}}+m_{\tilde{t}_{1,2}}=\sqrt{s}(\Delta \sim 6 \%)$.

In the large $\tan \beta$ scenario (Fig. 3.11) with the same parameters as in Fig. 3.10 we find a smaller negative boundary and a larger positive boundary $(-5 \% \lesssim \Delta \lesssim 7 \%)$ than in Fig. 3.10 . Here again these values are a nearby singularity effect at $m_{t}=m_{\tilde{\chi}_{1}^{+}}+m_{\tilde{b}_{2}}(\Delta \sim-5 \%)$ and a nearby threshold effect at $m_{\tilde{\chi}_{1}^{+}}+m_{\tilde{\chi}_{1}^{+}}=\sqrt{s}(\Delta \sim 7 \%)$. The large dip (dashed line) is again a threshold effect corresponding to $m_{\tilde{\chi}_{1}^{+}}+m_{\tilde{\chi}_{2}^{+}}=\sqrt{s}$ from the crossed chargino box. 


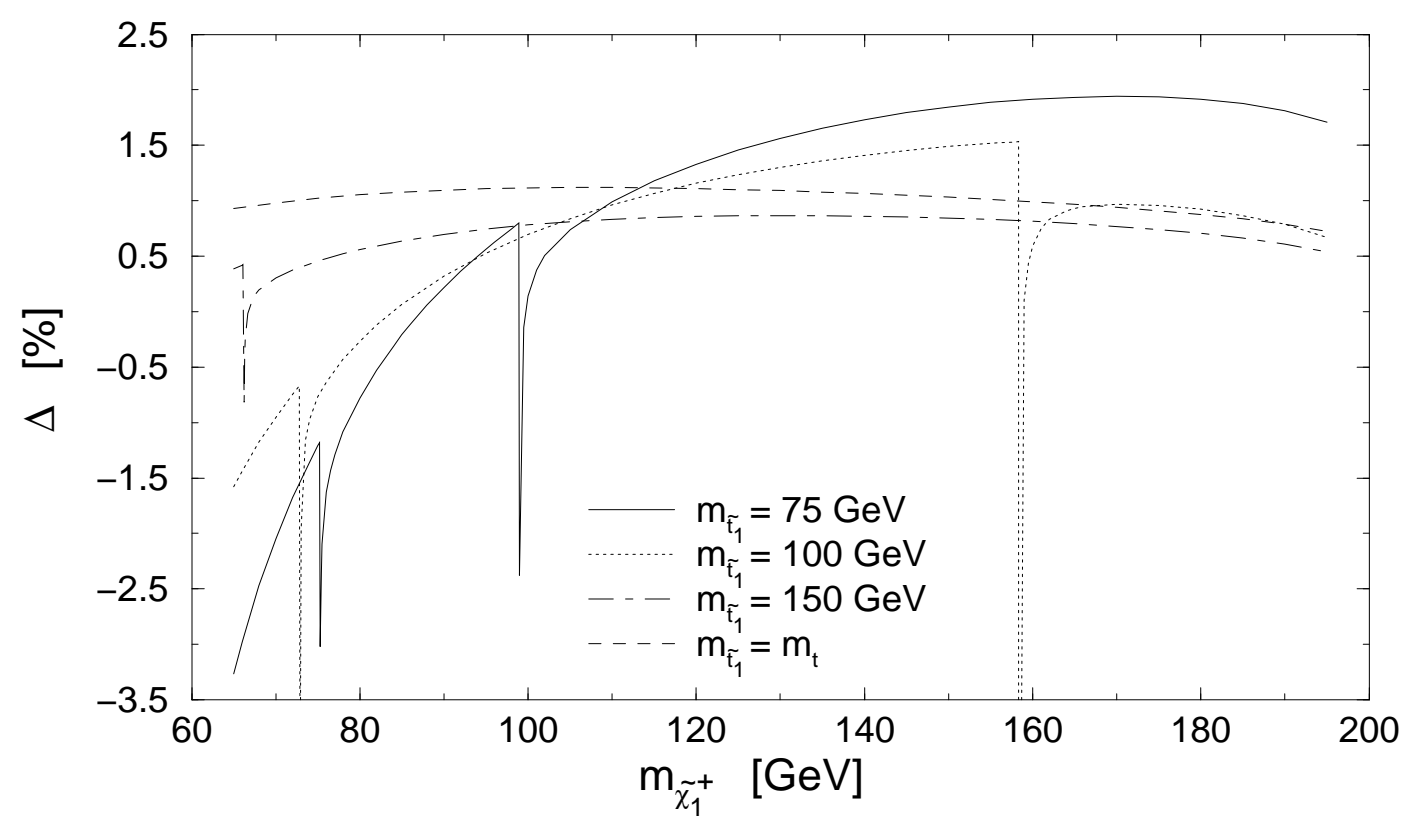

Figure 3.9: $e^{+} e^{-} \rightarrow t \bar{t}$. The $m_{\tilde{\chi}_{1}^{+}}$dependence of $\Delta$ for $\sqrt{s}=500 \mathrm{GeV}, \tan \beta=1.6, M_{A}=250$ $\mathrm{GeV}, \mu=-200 \mathrm{GeV}$ and $A=m_{\tilde{t}_{1}}$.

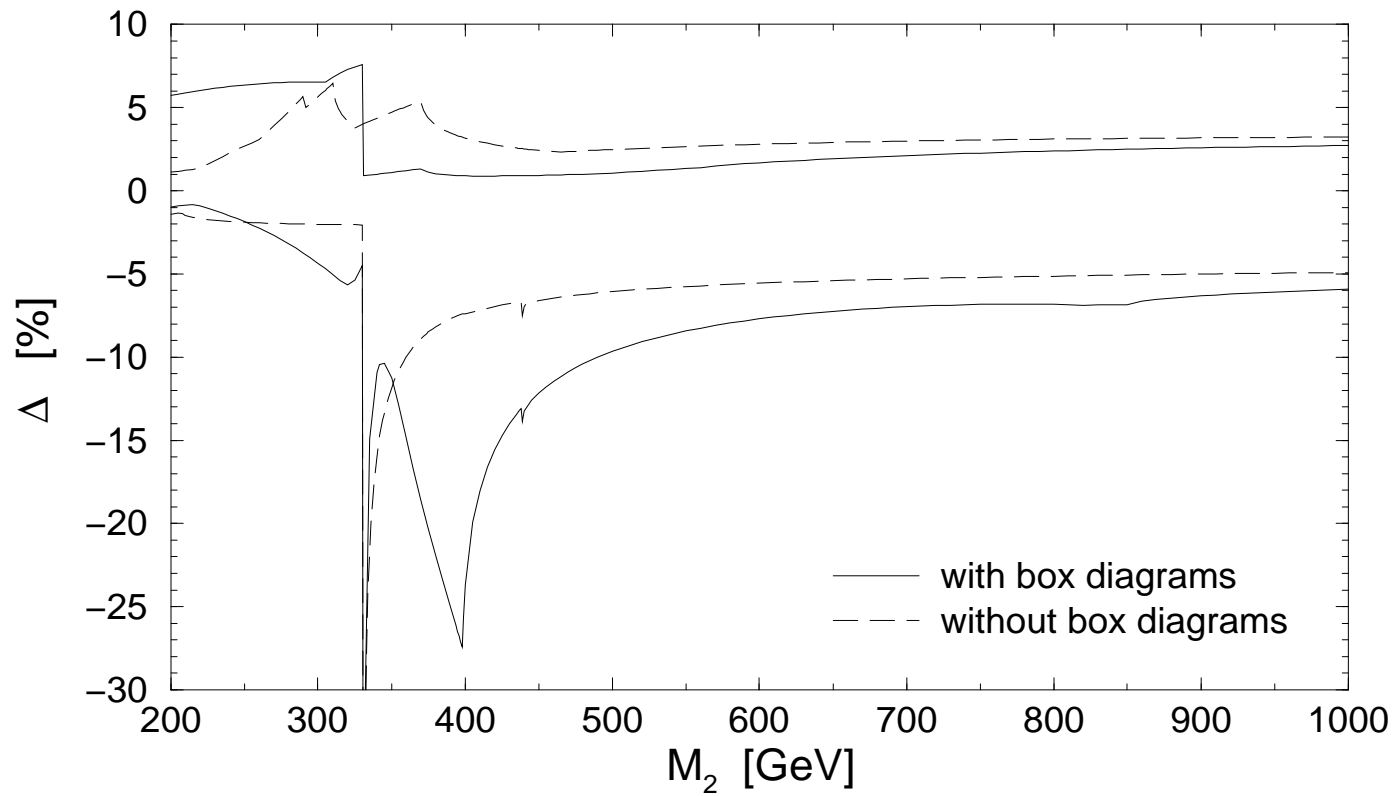

Figure 3.10: $e^{+} e^{-} \rightarrow t \bar{t}$. Predicted range of $\Delta$ for $\sqrt{s}=500 \mathrm{GeV}, \tan \beta=1.6, M_{A}=500 \mathrm{GeV}$, $100 \mathrm{GeV} \leq m_{\tilde{t}_{1}}=A \leq 500 \mathrm{GeV}$ and $-500 \mathrm{GeV} \leq \mu \leq 500 \mathrm{GeV}$ (but $-100 \mathrm{GeV}<\mu<100 \mathrm{GeV}$ is excluded). The predicted range is between the two upper and the two lower lines. 


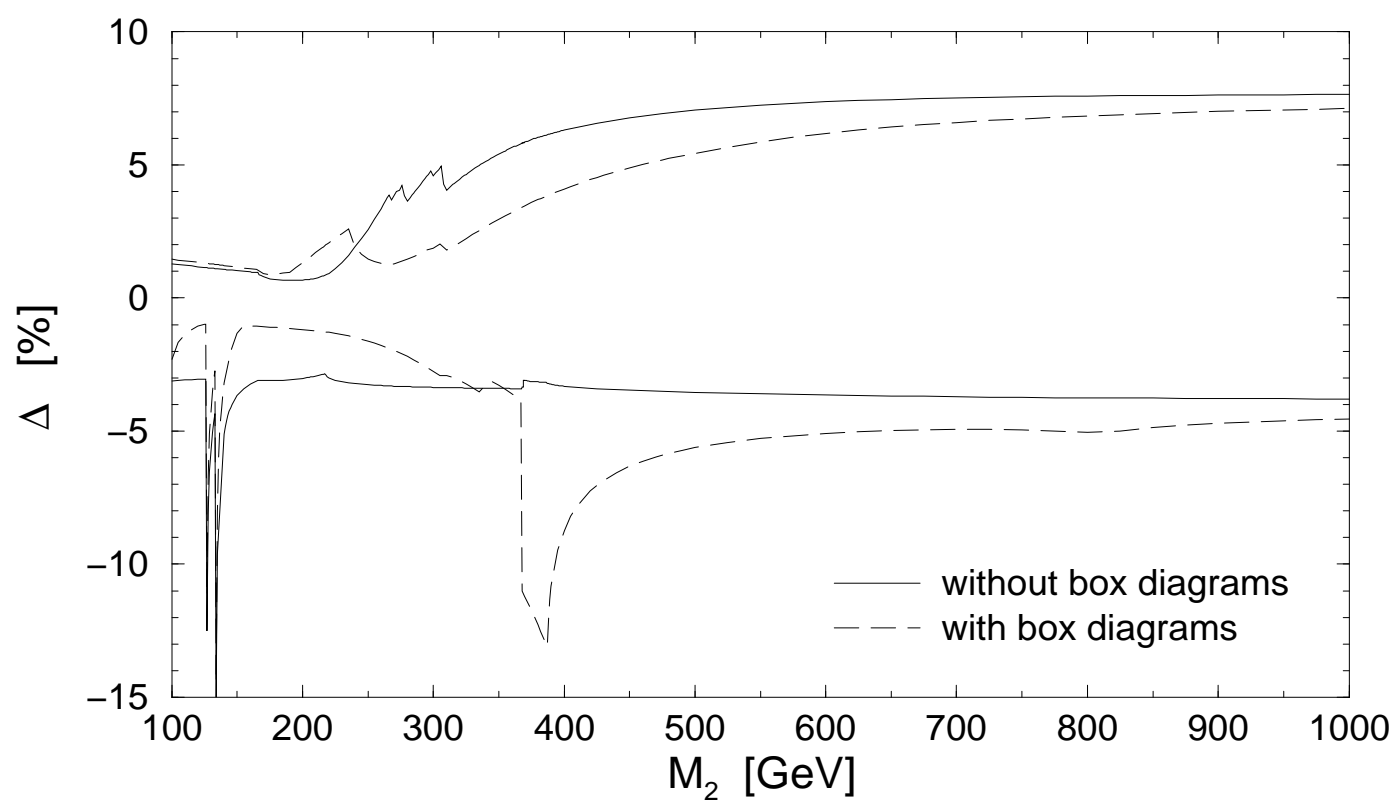

Figure 3.11: $e^{+} e^{-} \rightarrow t \bar{t}$. Predicted range of $\Delta$ for $\sqrt{s}=500 \mathrm{GeV}, \tan \beta=40, M_{A}=500 \mathrm{GeV}$, $100 \mathrm{GeV} \leq m_{\tilde{t}_{1}}=A \leq 500 \mathrm{GeV}$ and $-500 \mathrm{GeV} \leq \mu \leq 500 \mathrm{GeV}$ (but $-100 \mathrm{GeV}<\mu<100 \mathrm{GeV}$ is excluded). The predicted range is between the two upper and the two lower lines.

\section{Summary}

In this paper we have calculated the electroweak and SUSY-QCD 1-loop corrections to the process $e^{+} e^{-} \rightarrow f \bar{f}(f=\mu, \tau, q)$ in the MSSM.

Quantitative numerical studies of the size of the virtual SUSY effects have been performed for lepton pair and light quark pair production for LEP energies, and for top quark pair production at $500 \mathrm{GeV}$. Thereby, the parameters of the MSSM have been varied over a wide range in order to get an overall impression of the size of virtual SUSY effects.

For the light fermions, the virtual SUSY contributions in the cross sections amount to a few percent in the LEP region; they could be detectable for close-by SUSY states.

In the cross section for top production they can be larger, but typically stay below $10 \%$ (with exception of the peaks at some thresholds). In general, the effects of box diagrams are sizeable, mainly the SM box graphs. For the top case, also the SUSY box diagrams yield significant contributions.

For the forward-backward and left-right asymmetries no significant deviations between the MSSM and the SM occur.

\section{Acknowledgments}

We want to thank A. Kraft and S. Heinemeyer for helpful discussions. 


\section{A Masses and mixing states of the MSSM}

\section{A.1 The Higgs sector}

The neutral Higgs sector is conventionally fixed by choosing a value for $\tan \beta:=\frac{v_{2}}{v_{1}}$ and for the mass $M_{A^{0}}$ of the $\mathcal{C P}$-odd neutral Higgs boson $A^{0}$.

Radiative corrections for the neutral Higgs masses are important and dominated by terms proportional to $m_{t}^{4}$ [25]. We take them into account in the $m_{t}^{4}$-approximation following Ref. [17].

There are only small radiative corrections for the charged Higgs masses and the following equation holds only for $M_{A^{0}} \sim \mathcal{O}\left(M_{W}\right)$ [26] (we already used here the values $N_{C}^{f}=3, N_{G}=3$ and $N_{H}=2$, which are denoting the number of colors, families and Higgs doublets, respectively):

$$
\begin{aligned}
m_{H^{ \pm}, \mathrm{eff}}^{2}= & \frac{3 g_{2}^{2}}{32 \pi^{2} M_{W}^{2}}\left[\frac{2 m_{t}^{2} m_{b}^{2}}{\sin ^{2} \beta \cos ^{2} \beta}-M_{W}^{2}\left(\frac{m_{t}^{2}}{\sin ^{2} \beta}+\frac{m_{b}^{2}}{\cos ^{2} \beta}\right)+\frac{2}{3} M_{W}^{4}\right] \ln \frac{M_{S}^{2}}{m_{t}^{2}} \\
& +M_{A^{0}}^{2}+M_{W}^{2}+\frac{15 g_{1}^{2} M_{W}^{2}}{48 \pi^{2}} \ln \frac{M_{S}^{2}}{M_{W}^{2}}
\end{aligned}
$$

$M_{S}$ is a soft SUSY breaking parameter defined in Eq. (A.2) and $g_{1}, g_{2}$ denotes the $U(1), S U(2)$ gauge coupling, respectively.

\section{A.2 Sfermions}

For the simplest case we assume equal soft SUSY breaking parameters for all sfermions (motivated by minimal SUGRA)

$$
\mathbf{M}_{\tilde{\mathbf{Q}}}^{2}=\mathbf{M}_{\tilde{\mathbf{U}}}^{2}=\mathbf{M}_{\tilde{\mathbf{D}}}^{2}=\mathbf{M}_{\tilde{\mathbf{L}}}^{2}=\mathbf{M}_{\tilde{\mathbf{E}}}^{2}=M_{S}^{2} \mathbb{1}, \quad \mathbf{A}_{\mathbf{U}}=A_{u} \mathbb{1}, \quad \mathbf{A}_{\mathbf{L}}=\mathbf{A}_{\mathbf{D}}=A_{d} \mathbb{1}
$$

Then, the sfermion mass matrix is given by

$$
\left(\begin{array}{cc}
M_{S}^{2}+M_{Z}^{2} \cos 2 \beta\left(I_{3}^{f}-Q_{f} s_{w}^{2}\right)+m_{f}^{2} & m_{f}\left(A_{\{u ; d\}}-\mu\{\cot \beta ; \tan \beta\}\right) \\
m_{f}\left(A_{\{u ; d\}}-\mu\{\cot \beta ; \tan \beta\}\right) & M_{S}^{2}+M_{Z}^{2} \cos 2 \beta Q_{f} s_{w}^{2}+m_{f}^{2}
\end{array}\right)
$$

where $\{\cot \beta ; \tan \beta\}$ refer to the corresponding $\{u ; d\}$-type fermions.

In the case of mixed sfermions the left- and right-handed sfermions must be replaced by mass eigenstates of the sfermion mass matrix (A.3) [5]:

$$
\begin{aligned}
& \tilde{f}_{1}=\tilde{f}_{L} \cos \tilde{\theta}_{f}+\tilde{f}_{R} \sin \tilde{\theta}_{f}, \\
& \tilde{f}_{2}=\tilde{f}_{R} \cos \tilde{\theta}_{f}-\tilde{f}_{L} \sin \tilde{\theta}_{f},
\end{aligned}
$$

where $\tilde{\theta}_{f}$ denotes the mixing angle.

\footnotetext{
${ }^{5}$ Since we ignore mixing between sfermions of different generations, the $(3 \times 3)$ matrices $\mathbf{M}_{\tilde{\mathbf{Q}}}^{2}, \mathbf{M}_{\tilde{\mathbf{U}}}^{2}, \mathbf{M}_{\tilde{\mathbf{D}}}^{2}, \mathbf{M}_{\tilde{\mathbf{L}}}^{2}$, $\mathbf{M}_{\tilde{\mathbf{E}}}^{2}, \mathbf{A}_{\mathbf{U}}, \mathbf{A}_{\mathbf{D}}$ and $\mathbf{A}_{\mathbf{L}}$ are diagonal. Furthermore we chose all soft breaking parameters to be real.
} 


\section{A.3 Charginos}

The chargino mass eigenstates are obtained by diagonalizing the chargino mass matrix [3, 迎

$$
\mathbf{X}=\left(\begin{array}{cc}
M_{2} & \sqrt{2} M_{W} \sin \beta \\
\sqrt{2} M_{W} \cos \beta & \mu
\end{array}\right),
$$

containing the $S U(2)$ gaugino mass parameter $M_{2}$ with the help of two unitary $(2 \times 2)$ matrices $\mathbf{U}$ and $\mathbf{V}$ :

$$
\mathbf{U}^{*} \mathbf{X} \mathbf{V}^{-1}=\operatorname{diag}\left(m_{\tilde{\chi}_{1}^{+}}, m_{\tilde{\chi}_{2}^{+}}\right) .
$$

The squared entries in the diagonalized chargino mass matrix are obeying

$$
m_{\tilde{\chi}_{1,2}^{+}}^{2}=\frac{1}{2}\left\{M_{2}^{2}+\mu^{2}+2 M_{W}^{2} \mp\left[\left(M_{2}^{2}-\mu^{2}\right)^{2}+4 M_{W}^{4}\left(\cos ^{2} 2 \beta+M_{2}^{2}+\mu^{2}+2 \mu M_{2} \sin 2 \beta\right)\right]^{\frac{1}{2}}\right\} .
$$

\section{A.4 Neutralinos}

The four neutralino mass eigenstates are obtained from diagonalizing the $(4 \times 4)$ neutralino mass matrix [3, 田]

$$
\mathbf{Y}=\left(\begin{array}{cccc}
M_{1} & 0 & -M_{Z} s_{w} \cos \beta & M_{Z} s_{w} \sin \beta \\
0 & M_{2} & M_{Z} c_{w} \cos \beta & -M_{Z} c_{w} \sin \beta \\
-M_{Z} s_{w} \cos \beta & M_{Z} c_{w} \cos \beta & 0 & -\mu \\
M_{Z} s_{w} \sin \beta & -M_{Z} c_{w} \sin \beta & -\mu & 0
\end{array}\right)
$$

with the help of a unitary matrix $\mathbf{N}$ :

$$
\mathbf{N}^{*} \mathbf{Y} \mathbf{N}^{-1}=\operatorname{diag}\left(m_{\tilde{\chi}_{1}^{0}}, \ldots, m_{\tilde{\chi}_{4}^{0}}\right)
$$

The matrix $\mathbf{Y}$ contains the $U(1)$ gaugino mass parameter $M_{1}$ as a further input quantity. As conventionally done, we assume the SUSY-GUT constraints, with the gluino mass $m_{\tilde{g}} \equiv\left|M_{3}\right|$

$$
M_{1}=\frac{5}{3} \tan ^{2} \theta_{w} M_{2}, \quad M_{3}=\frac{\alpha_{s}}{\alpha} s_{w}^{2} M_{2},
$$

if not stated differently.

Note that for our calculation, the matrices $\mathbf{U}, \mathbf{V}$ and $\mathbf{N}$ have been chosen to be real. 


\section{B Self-energies}

This appendix contains the analytical expressions for the 1-loop self-energies of vector bosons and fermions. We make use of the following notations: The isospin partner of $f(\tilde{f})$ is denoted by $f^{\prime}\left(\tilde{f}^{\prime}\right)$. Fermions with $I_{3}= \pm 1 / 2$ are denoted $f^{ \pm}$, and sfermions by $\tilde{f}^{ \pm}$, analogously. The abbreviation 2HD denotes the SM sector plus the Two Higgs Doublet sector of the MSSM.

The Feynman rules of the MSSM are given in Refs. [3, 4, 5]. Note that the covariant derivative given there is not compatible with the one used in this paper:

$$
D_{\mu}=\partial_{\mu}-i \frac{1}{2} g_{2} \sigma_{l} W_{\mu}^{l}+i \frac{1}{2} g_{1} Y B_{\mu} .
$$

So we had to change the Feynman rules by substituting $\sin \theta_{w}$ (defined in Eq. (2.21) with $-\sin \theta_{w}$ and in addition we had to provide every Higgs field with a factor $(-1)$.

\section{B.1 Vector boson self-energies}

The vector boson self-energies are separated in 2HD-, sfermion-, and chargino/neutralino contributions. The momenta and internal masses are illustrated in Figure B.1. As a short hand notation we introduce the functions $F_{1}, F_{2}$ (which enter the fermion, chargino and neutralino loop contribution to the vector boson self-energies):

$$
\begin{aligned}
F_{1}\left(p^{2}, m_{1}, m_{2}, a, b, a^{\prime}, b^{\prime}\right)= & 8\left\{( a a ^ { \prime } + b b ^ { \prime } ) \left[-2 B_{22}\left(p^{2}, m_{1}, m_{2}\right)+A_{0}\left(m_{2}\right)+m_{1}^{2} B_{0}\left(p^{2}, m_{1}, m_{2}\right)\right.\right. \\
& \left.\left.+p^{2} B_{1}\left(p^{2}, m_{1}, m_{2}\right)\right]-\left(a b^{\prime}+b a^{\prime}\right) m_{1} m_{2} B_{0}\left(p^{2}, m_{1}, m_{2}\right)\right\}, \\
F_{2}\left(p^{2}, m_{1}, m_{2}\right)= & 10 B_{22}\left(p^{2}, m_{1}, m_{2}\right)+\left[4 p^{2}+m_{1}^{2}+m_{2}^{2}\right] B_{0}\left(p^{2}, m_{1}, m_{2}\right)+A_{0}\left(m_{1}\right) \\
& +A_{0}\left(m_{2}\right)-2\left[m_{1}^{2}+m_{2}^{2}-\frac{p^{2}}{3}\right],
\end{aligned}
$$

with the 1- and 2-point functions $A_{0}, B_{0}, B_{1}$ and $B_{22}$ from Ref. [27], in the convention of Refs. [13, 15].

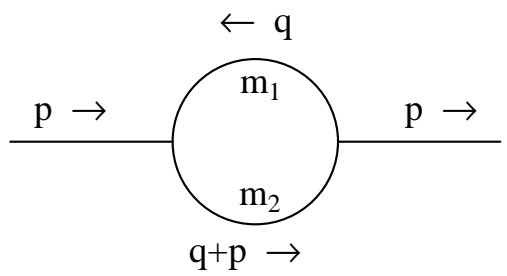

Figure B.1: Masses and momenta for the vector boson self-energies.

The self-energies are obtained as the sum of the terms depicted below with their analytical expression. 


\section{B.1.1 Photon self-energy}
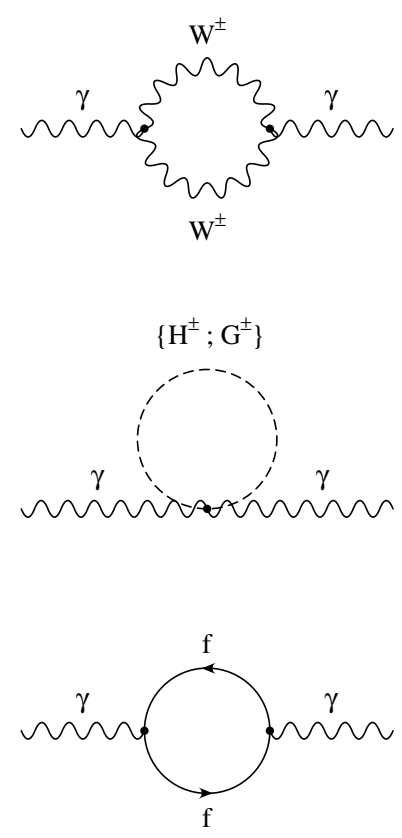

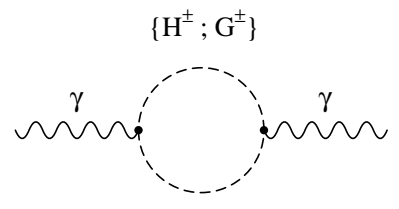

$\left\{\mathrm{H}^{ \pm} ; \mathrm{G}^{ \pm}\right\}$

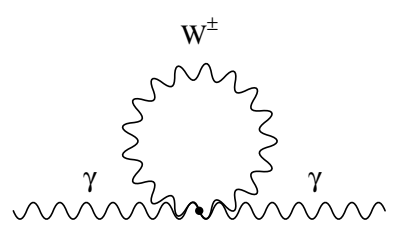

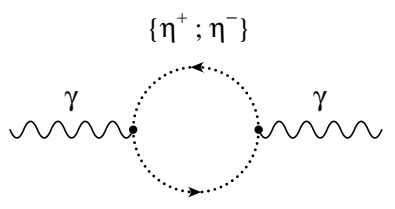

$\left\{\eta^{+} ; \eta^{-}\right\}$

$\left\{\mathrm{G}^{+} ; \mathrm{G}^{-}\right\}$

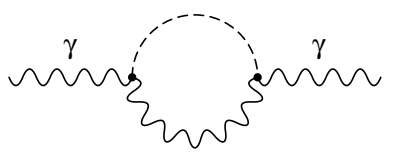

$\left\{\mathrm{W}^{+} ; \mathrm{W}^{-}\right\}$

$$
\begin{aligned}
\Sigma_{2 H D}^{\gamma \gamma}\left(p^{2}\right)= & -\frac{\alpha}{4 \pi}\left\{4 B_{22}\left(p^{2}, m_{H^{ \pm}}, m_{H^{ \pm}}\right)+2 B_{22}\left(p^{2}, M_{W}, M_{W}\right)\right. \\
& -2 M_{W}^{2} B_{0}\left(p^{2}, M_{W}, M_{W}\right)-2 A_{0}\left(m_{H^{ \pm}}\right)-8 A_{0}\left(M_{W}\right)+4 M_{W}^{2} \\
& \left.+F_{2}\left(p^{2}, M_{W}, M_{W}\right)+\sum_{f} N_{C}^{f} Q_{f}^{2} F_{1}\left(p^{2}, m_{f}, m_{f}, \frac{1}{2}, \frac{1}{2}, \frac{1}{2}, \frac{1}{2}\right)\right\},
\end{aligned}
$$
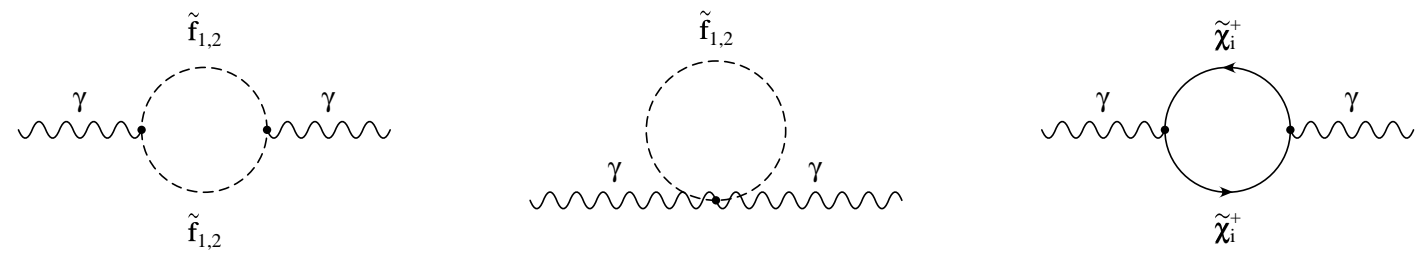

$$
\begin{aligned}
\Sigma_{\tilde{f}}^{\gamma \gamma}\left(p^{2}\right)= & -\frac{\alpha}{4 \pi} 2 \sum_{f} N_{C}^{f} Q_{f}^{2}\left\{2 B_{22}\left(p^{2}, m_{\tilde{f}_{1}}, m_{\tilde{f}_{1}}\right)+2 B_{22}\left(p^{2}, m_{\tilde{f}_{2}}, m_{\tilde{f}_{2}}\right)\right. \\
& \left.-A_{0}\left(m_{\tilde{f}_{1}}\right)-A_{0}\left(m_{\tilde{f}_{2}}\right)\right\} \\
\Sigma_{\tilde{\chi}}^{\gamma \gamma}\left(p^{2}\right)= & -\frac{\alpha}{4 \pi} \sum_{i=1}^{2} F_{1}\left(p^{2}, m_{\tilde{\chi}_{i}^{+}}, m_{\tilde{\chi}_{i}^{+}}, \frac{1}{2}, \frac{1}{2}, \frac{1}{2}, \frac{1}{2}\right) .
\end{aligned}
$$




\section{B.1.2 $Z$ self-energy}

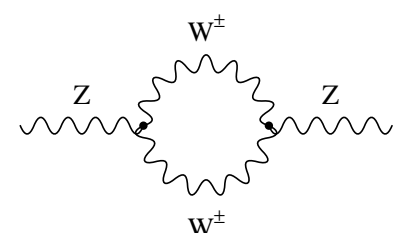

$\left\{h^{0} ; H^{0} ; A^{0} ; H^{ \pm} ; G^{0} ; G^{ \pm}\right\}$
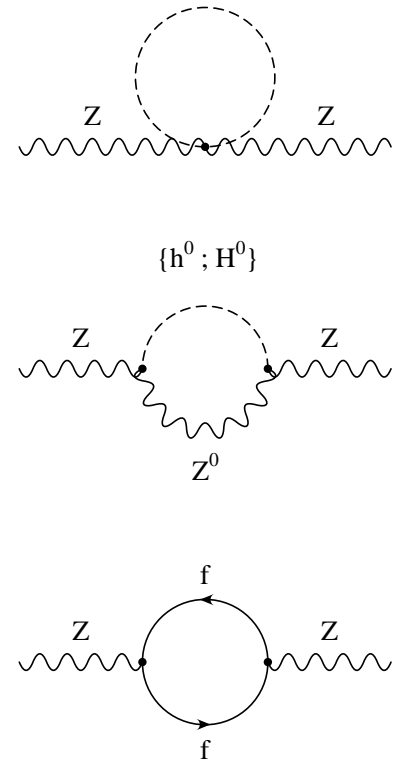

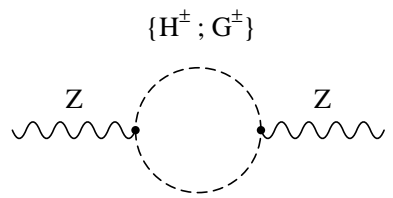

$\left\{\mathrm{H}^{ \pm} ; \mathrm{G}^{ \pm}\right\}$
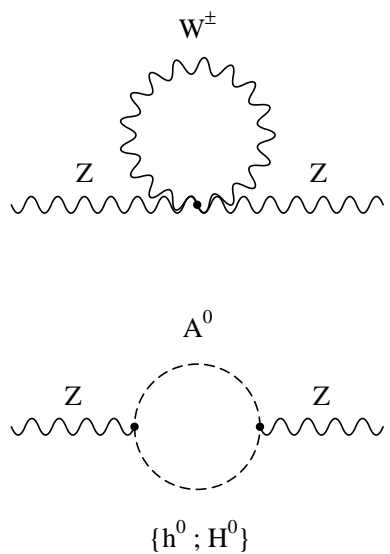

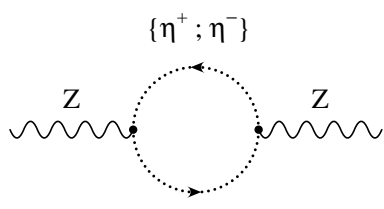

$\left\{\eta^{+} ; \eta^{-}\right\}$

$\left\{\mathrm{G}^{+} ; \mathrm{G}^{-}\right\}$

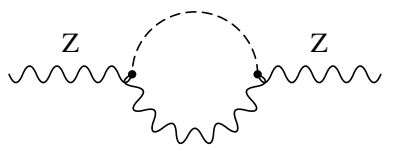

$\left\{\mathrm{W}^{+} ; \mathrm{W}^{-}\right\}$

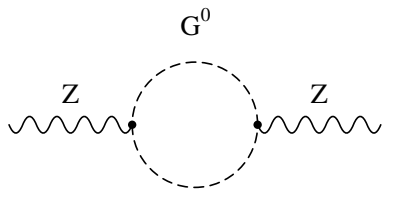

$\left\{h^{0} ; H^{0}\right\}$

$$
\begin{aligned}
\Sigma_{2 H D}^{Z Z}\left(p^{2}\right)= & \frac{\alpha}{4 \pi} \frac{1}{s_{w}^{2} c_{w}^{2}}\left\{-\sin ^{2}(\beta-\alpha)\left[B_{22}\left(p^{2}, M_{A^{0}}, m_{H^{0}}\right)+B_{22}\left(p^{2}, M_{Z}, m_{h^{0}}\right)\right]\right. \\
& -\cos ^{2}(\beta-\alpha)\left[B_{22}\left(p^{2}, M_{A^{0}}, m_{h^{0}}\right)+B_{22}\left(p^{2}, M_{Z}, m_{H^{0}}\right)\right] \\
& -\cos ^{2} 2 \theta_{w}\left[B_{22}\left(p^{2}, m_{H^{ \pm}}, m_{H^{ \pm}}\right)+B_{22}\left(p^{2}, M_{W}, M_{W}\right)\right] \\
& +2 c_{w}^{4} B_{22}\left(p^{2}, M_{W}, M_{W}\right)+\frac{1}{4}\left[A_{0}\left(m_{h^{0}}\right)+A_{0}\left(m_{H^{0}}\right)+A_{0}\left(M_{A^{0}}\right)+A_{0}\left(M_{Z}\right)\right] \\
& +\frac{\cos ^{2} 2 \theta_{w}}{2}\left[A_{0}\left(M_{W}\right)+A_{0}\left(m_{H^{ \pm}}\right)\right]+M_{Z}^{2}\left[2 s_{w}^{4} c_{w}^{2} B_{0}\left(p^{2}, M_{W}, M_{W}\right)\right. \\
& \left.+\sin ^{2}(\beta-\alpha) B_{0}\left(p^{2}, m_{h^{0}}, M_{Z}\right)+\cos ^{2}(\beta-\alpha) B_{0}\left(p^{2}, m_{H^{0}}, M_{Z}\right)\right] \\
& +c_{w}^{4}\left[6 A_{0}\left(M_{W}\right)-4 M_{W}^{2}\right]-c_{w}^{4} F_{2}\left(p^{2}, M_{W}, M_{W}\right) \\
& \left.-s_{w}^{2} c_{w}^{2} \sum_{f} N_{C}^{f} F_{1}\left(p^{2}, m_{f}, m_{f}, \frac{v_{f}+a_{f}}{2}, \frac{v_{f}-a_{f}}{2}, \frac{v_{f}+a_{f}}{2}, \frac{v_{f}-a_{f}}{2}\right)\right\},
\end{aligned}
$$



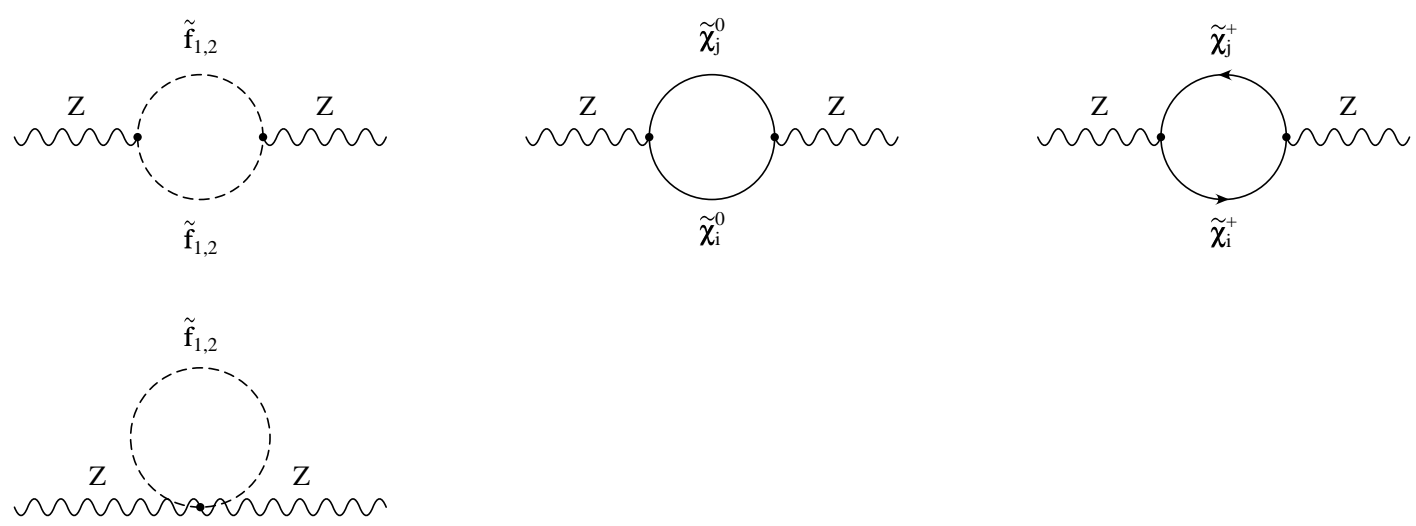

$$
\begin{aligned}
\Sigma_{\tilde{f}}^{Z Z}\left(p^{2}\right)= & -\frac{\alpha}{4 \pi} \sum_{f} N_{C}^{f}\left\{\frac{c_{\tilde{\theta}}^{2} s_{\tilde{\theta}}^{2}}{s_{w}^{2} c_{w}^{2}}\left[B_{22}\left(p^{2}, m_{\tilde{f}_{1}}, m_{\tilde{f}_{2}}\right)+B_{22}\left(p^{2}, m_{\tilde{f}_{2}}, m_{\tilde{f}_{1}}\right)\right]\right. \\
& +\frac{4\left(I_{3}^{f} c_{\tilde{\theta}}^{2}-Q_{f} s_{w}^{2}\right)^{2}}{s_{w}^{2} c_{w}^{2}} B_{22}\left(p^{2}, m_{\tilde{f}_{1}}, m_{\tilde{f}_{1}}\right)+\frac{4\left(I_{3}^{f} s_{\tilde{\theta}}^{2}-Q_{f} s_{w}^{2}\right)^{2}}{s_{w}^{2} c_{w}^{2}} B_{22}\left(p^{2}, m_{\tilde{f}_{2}}, m_{\tilde{f}_{2}}\right) \\
& \left.-2 \frac{\left(I_{3}^{f}-Q_{f} s_{w}^{2}\right)^{2} c_{\tilde{\tilde{\theta}}}^{2}+Q_{f}^{2} s_{w}^{4} s_{\tilde{\theta}}^{2}}{s_{w}^{2} c_{w}^{2}} A_{0}\left(m_{\tilde{f}_{1}}\right)-2 \frac{\left(I_{3}^{f}-Q_{f} s_{w}^{2}\right)^{2} s_{\tilde{\theta}}^{2}+Q_{f}^{2} s_{w}^{4} c_{\tilde{\theta}}^{2}}{s_{w}^{2} c_{w}^{2}} A_{0}\left(m_{\tilde{f}_{2}}\right)\right\} \\
\Sigma_{\tilde{\chi}}^{Z Z}\left(p^{2}\right)= & -\frac{\alpha}{4 \pi} \frac{1}{4 s_{w}^{2} c_{w}^{2}}\left\{\frac{1}{2} \sum_{i, j=1}^{4} F_{1}\left(p^{2}, m_{\tilde{\chi}_{j}^{0}}, m_{\tilde{\chi}_{i}^{0}}, O_{i j}^{\prime \prime L}, O_{i j}^{\prime \prime R}, O_{j i}^{\prime \prime L}, O_{j i}^{\prime \prime R}\right)\right. \\
& \left.+\sum_{i, j=1}^{2} F_{1}\left(p^{2}, m_{\tilde{\chi}_{j}^{+}}, m_{\tilde{\chi}_{i}^{+}}, O_{i j}^{\prime L}, O_{i j}^{\prime R}, O_{j i}^{\prime L}, O_{j i}^{\prime R}\right)\right\}
\end{aligned}
$$

with the vector- and axial vector couplings

$$
v_{f}=\frac{I_{3}^{f}-2 s_{w}^{2} Q_{f}}{2 s_{w} c_{w}}, \quad a_{f}=\frac{I_{3}^{f}}{2 s_{w} c_{w}},
$$

and $c_{\tilde{\theta}}:=\cos \tilde{\theta}_{f}, s_{\tilde{\theta}}:=\sin \tilde{\theta}_{f}$. The coefficients of the chargino- and neutralino couplings are given by the expressions

$$
\begin{aligned}
O_{i j}^{L L} & =-V_{i 1} V_{j 1}^{*}-\frac{1}{2} V_{i 2} V_{j 2}^{*}+\delta_{i j} s_{w}^{2}, \\
O_{i j}^{\prime R} & =-U_{i 1}^{*} U_{j 1}-\frac{1}{2} U_{i 2}^{*} U_{j 2}+\delta_{i j} s_{w}^{2}, \\
O_{i j}^{\prime \prime L} & =-\frac{1}{2} N_{i 3} N_{j 3}^{*}+\frac{1}{2} N_{i 4} N_{j 4}^{*}, \\
O_{i j}^{\prime \prime R} & =-O_{i j}^{\prime \prime L *},
\end{aligned}
$$

with the matrices $U_{i j}, V_{i j}$ for the charginos and $N_{i j}$ for the neutralinos, see Appendices A.3, A.4. 


\section{B.1.3 $W$ self-energy}

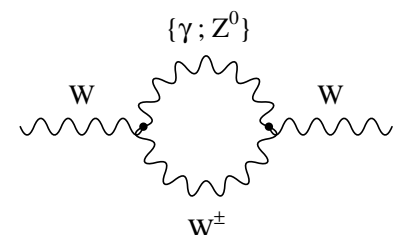

$\left\{\mathrm{h}^{0} ; \mathrm{H}^{0} ; \mathrm{A}^{0} ; \mathrm{H}^{ \pm} ; \mathrm{G}^{0} ; \mathrm{G}^{ \pm}\right\}$

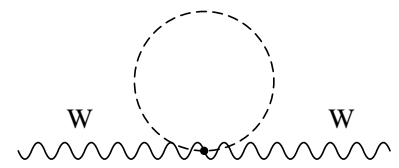

$\left\{\mathrm{h}^{0} ; \mathrm{H}^{0}\right\}$

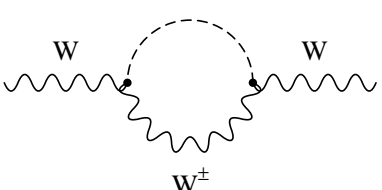

$\mathrm{W}^{ \pm}$

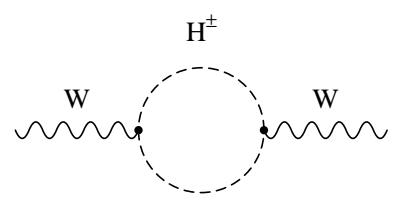

$\left\{\mathrm{h}^{0} ; \mathrm{H}^{0} ; \mathrm{A}^{0}\right\}$

$\left\{\gamma ; \mathrm{Z}^{0} ; \mathrm{W}^{ \pm}\right\}$
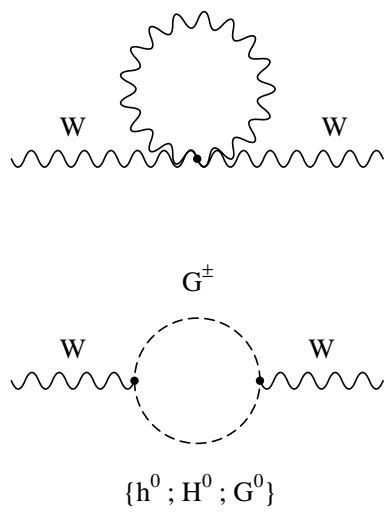

$\left\{\eta^{\gamma} ; \eta^{Z} ; \eta^{ \pm} ; \eta^{ \pm}\right\}$

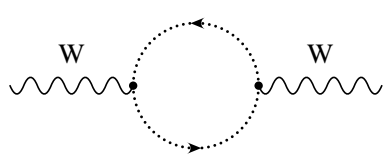

$\left\{\eta^{ \pm} ; \eta^{ \pm} ; \eta^{\gamma} ; \eta^{\mathrm{z}}\right\}$

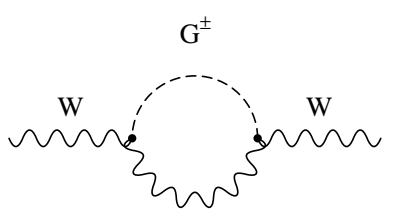

$\left\{\gamma ; Z^{0}\right\}$

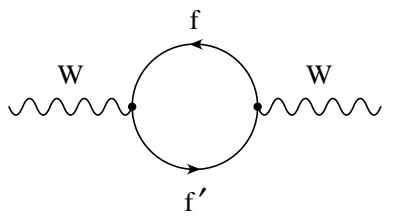

$$
\begin{aligned}
\Sigma_{2 H D}^{W W}\left(p^{2}\right)= & \frac{\alpha}{4 \pi} \frac{1}{s_{w}^{2}}\left\{-\sin ^{2}(\beta-\alpha)\left[B_{22}\left(p^{2}, m_{H^{ \pm}}, m_{H^{0}}\right)+B_{22}\left(p^{2}, M_{W}, m_{h^{0}}\right)\right]\right. \\
& -\cos ^{2}(\beta-\alpha)\left[B_{22}\left(p^{2}, m_{H^{ \pm}}, m_{h^{0}}\right)+B_{22}\left(p^{2}, M_{W}, m_{H^{0}}\right)\right] \\
& -B_{22}\left(p^{2}, M_{W}, M_{Z}\right)-B_{22}\left(p^{2}, m_{H^{ \pm}}, M_{A^{0}}\right) \\
& +2 s_{w}^{2} B_{22}\left(p^{2}, 0, M_{W}\right)+2 c_{w}^{2} B_{22}\left(p^{2}, M_{W}, M_{Z}\right) \\
& +\frac{1}{4}\left[A_{0}\left(m_{h^{0}}\right)+A_{0}\left(m_{H^{0}}\right)+A_{0}\left(M_{A^{0}}\right)+A_{0}\left(M_{Z}\right)+2 A_{0}\left(M_{W}\right)+2 A_{0}\left(m_{H^{ \pm}}\right)\right] \\
& +M_{W}^{2}\left[\sin ^{2}(\beta-\alpha) B_{0}\left(p^{2}, m_{h^{0}}, M_{W}\right)+\cos ^{2}(\beta-\alpha) B_{0}\left(p^{2}, m_{H^{0}}, M_{W}\right)\right. \\
& \left.+s_{w}^{2} B_{0}\left(p^{2}, M_{W}, 0\right)+\frac{s_{w}^{4}}{c_{w}^{2}} B_{0}\left(p^{2}, M_{W}, M_{Z}\right)\right] \\
& +3 A_{0}\left(M_{W}\right)-2 M_{W}^{2}+c_{w}^{2}\left[3 A_{0}\left(M_{Z}\right)-2 M_{Z}^{2}\right] \\
& -c_{w}^{2} F_{2}\left(p^{2}, M_{Z}, M_{W}\right)-s_{w}^{2} F_{2}\left(p^{2}, 0, M_{W}\right) \\
& \left.-\sum_{\text {doublets }} \frac{N_{C}^{f}}{2} F_{1}\left(p^{2}, m_{f}, m_{f^{-}}, \frac{1}{2}, 0, \frac{1}{2}, 0\right)\right\}
\end{aligned}
$$



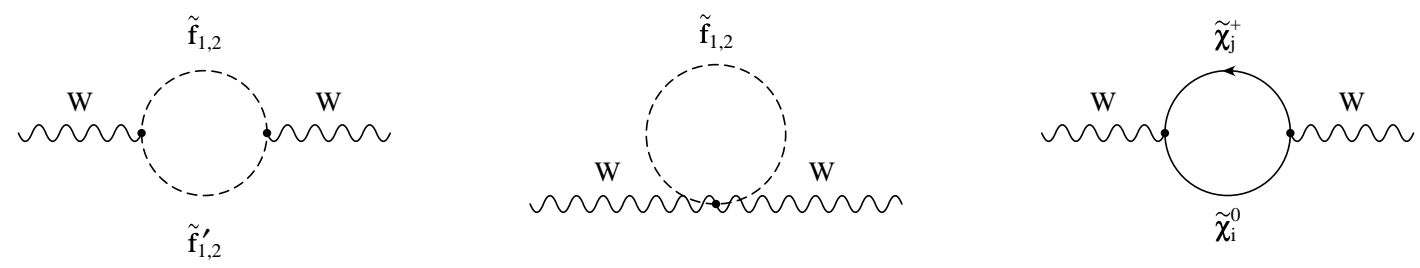

$$
\begin{aligned}
\sum_{\tilde{f}}^{W W}\left(p^{2}\right)= & -\frac{\alpha}{4 \pi} \frac{1}{s_{w}^{2}} \sum_{\text {doublets }} N_{C}^{f}\left\{2 c_{\tilde{\theta}^{+}}^{2} c_{\tilde{\theta}^{-}}^{2} B_{22}\left(p^{2}, m_{\tilde{f}_{1}^{+}}, m_{\tilde{f}_{1}^{-}}\right)\right. \\
& +2 c_{\tilde{\theta}^{+}}^{2} s_{\tilde{\theta}^{-}}^{2} B_{22}\left(p^{2}, m_{\tilde{f}_{1}^{+}}, m_{\tilde{f}_{2}^{-}}\right) \\
& \left.+2 s_{\tilde{\theta}^{+}}^{2} c_{\tilde{\theta}^{-}}^{2} B_{22}\left(p^{2}, m_{\tilde{f}_{2}^{+}}, m_{\tilde{f}_{1}^{-}}\right)+2 s_{\tilde{\theta}^{+}}^{2} s_{\tilde{\theta}^{-}}^{2} B_{22}\left(p^{2}, m_{\tilde{f}_{2}^{+}}, m_{\tilde{f}_{2}^{-}}\right)\right\} \\
& +\frac{\alpha}{4 \pi} \frac{1}{2 s_{w}^{2}} \sum_{f} N_{C}^{f}\left\{c_{\tilde{\theta}^{2}}^{2} A_{0}\left(m_{\tilde{f}_{1}}\right)+s_{\tilde{\theta}^{2}}^{2} A_{0}\left(m_{\tilde{f}_{2}}\right)\right\} \\
\sum_{\tilde{\chi}}^{W W}\left(p^{2}\right)= & -\frac{\alpha}{4 \pi} \frac{1}{4 s_{w}^{2}} \sum_{i=1}^{4} \sum_{j=1}^{2} F_{1}\left(p^{2}, m_{\tilde{\chi}_{j}^{+}}, m_{\tilde{\chi}_{i}^{0}}, O_{i j}^{L}, O_{i j}^{R}, O_{i j}^{L *}, O_{i j}^{R *}\right),
\end{aligned}
$$

with

$$
\begin{aligned}
O_{i j}^{L} & =-\frac{1}{\sqrt{2}} N_{i 4} V_{j 2}^{*}+N_{i 2} V_{j 1}^{*}, \\
O_{i j}^{R} & =+\frac{1}{\sqrt{2}} N_{i 3}^{*} U_{j 2}+N_{i 2}^{*} U_{j 1},
\end{aligned}
$$

containing the matrices $U_{i j}, V_{i j}, N_{i j}$ for the chargino and neutralino sector, see Appendices A.3, A.4. 


\section{B.1.4 Photon- $Z$ mixing}
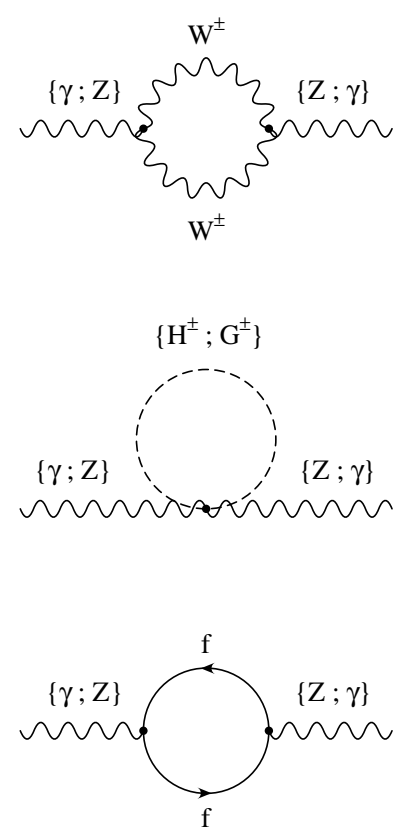

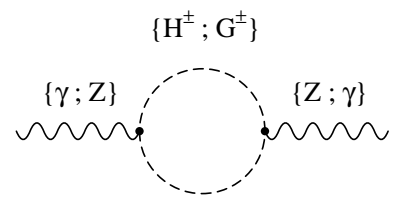

$\left\{\mathrm{H}^{ \pm} ; \mathrm{G}^{ \pm}\right\}$

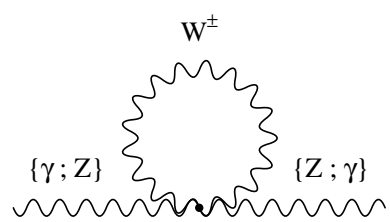

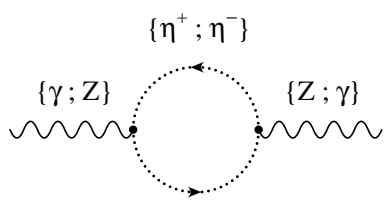

$\left\{\eta^{+} ; \eta^{-}\right\}$

$\left\{\mathrm{G}^{+} ; \mathrm{G}^{-}\right\}$

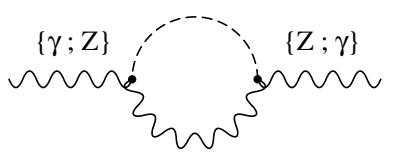

$\left\{\mathrm{W}^{+} ; \mathrm{W}^{-}\right\}$

$$
\begin{aligned}
\Sigma_{2 H D}^{\gamma Z}\left(p^{2}\right)= & -\frac{\alpha}{4 \pi}\left\{-\frac{2 \cos 2 \theta_{w}}{s_{w} c_{w}}\left[B_{22}\left(p^{2}, m_{H^{ \pm}}, m_{H^{ \pm}}\right)+B_{22}\left(p^{2}, M_{W}, M_{W}\right)\right]\right. \\
& +\frac{2 c_{w}}{s_{w}} B_{22}\left(p^{2}, M_{W}, M_{W}\right)-2 s_{w} c_{w} M_{Z}^{2} B_{0}\left(p^{2}, M_{W}, M_{W}\right) \\
& \left.+\frac{\cos 2 \theta_{w}}{s_{w} c_{w}}\left[A_{0}\left(m_{H^{ \pm}}\right)+A_{0}\left(M_{W}\right)\right]+\frac{c_{w}}{s_{w}}\left[6 A_{0}\left(M_{W}\right)-4 M_{W}^{2}\right)\right] \\
& -\frac{c_{w}}{s_{w}} F_{2}\left(p^{2}, M_{W}, M_{W}\right) \\
& \left.-\sum_{f} N_{C}^{f} Q_{f} F_{1}\left(p^{2}, m_{f}, m_{f}, \frac{1}{2}, \frac{1}{2}, \frac{v_{f}-a_{f}}{2}, \frac{v_{f}+a_{f}}{2}\right)\right\}
\end{aligned}
$$



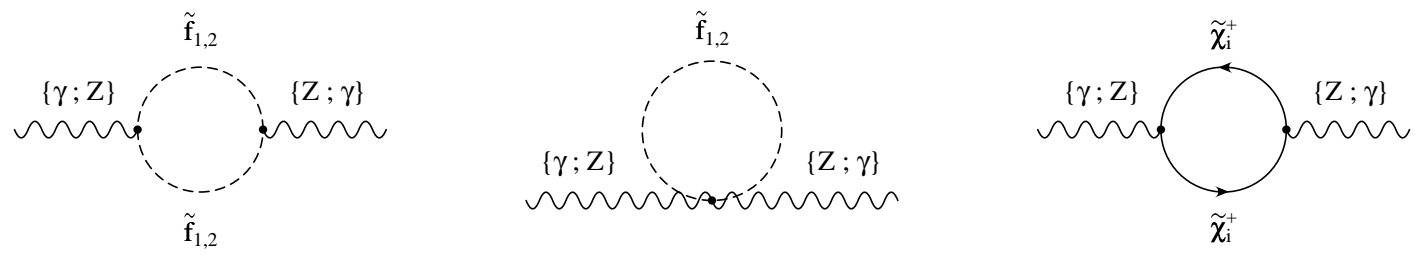

$$
\begin{aligned}
\Sigma_{\tilde{f}}^{\gamma Z}\left(p^{2}\right)= & \frac{\alpha}{4 \pi} 2 \sum_{f} N_{C}^{f} Q_{f}\left\{\frac{I_{3}^{f} c_{\tilde{\theta}}^{2}-Q_{f} s_{w}^{2}}{s_{w} c_{w}}\left[2 B_{22}\left(p^{2}, m_{\tilde{f}_{1}}, m_{\tilde{f}_{1}}\right)-A_{0}\left(m_{\tilde{f}_{1}}\right)\right]\right. \\
& \left.+\frac{I_{3}^{f} s_{\tilde{\theta}}^{2}-Q_{f} s_{w}^{2}}{s_{w} c_{w}}\left[2 B_{22}\left(p^{2}, m_{\tilde{f}_{2}}, m_{\tilde{f}_{2}}\right)-A_{0}\left(m_{\tilde{f}_{2}}\right)\right]\right\} \\
\Sigma_{\tilde{\chi}}^{\gamma Z}\left(p^{2}\right)= & -\frac{\alpha}{4 \pi} \frac{1}{2 s_{w} c_{w}} \sum_{i=1}^{2} F_{1}\left(p^{2}, m_{\tilde{\chi}_{i}^{+}}, m_{\tilde{\chi}_{i}^{+}}, \frac{1}{2}, \frac{1}{2}, O_{i i}^{L}, O_{i i}^{\prime R}\right) .
\end{aligned}
$$

\section{B.2 Fermion self-energies}

The fermion self-energy can be expressed in terms of the scalar functions $\Sigma_{\{S, V, A\}}^{f}$ as coefficients for the scalar-, vector-, and axial vector part:

$$
\Sigma^{f}(p)=\not p \Sigma_{V}^{f}\left(p^{2}\right)+\not p \gamma_{5} \Sigma_{A}^{f}\left(p^{2}\right)+m_{f} \Sigma_{S}^{f}\left(p^{2}\right) .
$$

The contributions are separated in a (weak) 2HD part, a (weak) SUSY part and a gluino part. The momenta and internal masses are illustrated in figure B.2. For computing the fermionnumber-violating vertices the algorithm of Ref. [28] is utilized. The arrows within the lines denote the charge flow and long, thin arrows denote the fermion flow.

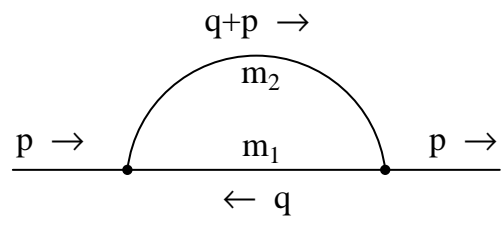

Figure B.2: Masses and momenta for the fermion self-energies. 
We introduce the following definitions and shorthand notations:

$$
\begin{aligned}
& X_{i 1}^{+\{d ; u\}}:=-\frac{1}{s_{w}}\left\{V_{i 1} ; U_{i 1}\right\} \cos \tilde{\theta}_{f^{\prime}}+\frac{m_{f^{\prime}}}{\sqrt{2} M_{W} s_{w}}\left\{\frac{V_{i 2}}{\sin \beta} ; \frac{U_{i 2}}{\cos \beta}\right\} \sin \tilde{\theta}_{f^{\prime}}, \\
& X_{i 2}^{+\{d ; u\}}:=+\frac{1}{s_{w}}\left\{V_{i 1} ; U_{i 1}\right\} \sin \tilde{\theta}_{f^{\prime}}+\frac{m_{f^{\prime}}}{\sqrt{2} M_{W} s_{w}}\left\{\frac{V_{i 2}}{\sin \beta} ; \frac{U_{i 2}}{\cos \beta}\right\} \cos \tilde{\theta}_{f^{\prime}}, \\
& Y_{i 1}^{+\{d ; u\}}:=+\frac{m_{f}}{\sqrt{2} M_{W} s_{w}}\left\{\frac{U_{i 2}}{\cos \beta} ; \frac{V_{i 2}}{\sin \beta}\right\} \cos \tilde{\theta}_{f^{\prime}}, \\
& Y_{i 2}^{+\{d ; u\}}:=-\frac{m_{f}}{\sqrt{2} M_{W} s_{w}}\left\{\frac{U_{i 2}}{\cos \beta} ; \frac{V_{i 2}}{\sin \beta}\right\} \sin \tilde{\theta}_{f^{\prime}}, \\
& X_{i 1}^{0\{d ; u\}}:=-\sqrt{2}\left[Q_{f} N_{i 1}^{\prime} \mp \frac{\frac{1}{2} \pm Q_{f} s_{w}^{2}}{s_{w} c_{w}} N_{i 2}^{\prime}\right] \cos \tilde{\theta}_{f}-\frac{m_{f}}{\sqrt{2} M_{W} s_{w}}\left\{\frac{N_{i 3}}{\cos \beta} ; \frac{N_{i 4}}{\sin \beta}\right\} \sin \tilde{\theta}_{f} \\
& X_{i 2}^{0\{d ; u\}}:=+\sqrt{2}\left[Q_{f} N_{i 1}^{\prime} \mp \frac{\frac{1}{2} \pm Q_{f} s_{w}^{2}}{s_{w} c_{w}} N_{i 2}^{\prime}\right] \sin \tilde{\theta}_{f}-\frac{m_{f}}{\sqrt{2} M_{W} s_{w}}\left\{\frac{N_{i 3}}{\cos \beta} ; \frac{N_{i 4}}{\sin \beta}\right\} \cos \tilde{\theta}_{f}, \\
& Y_{i 1}^{0\{d ; u\}}:=-\frac{m_{f}}{\sqrt{2} M_{W} s_{w}}\left\{\frac{N_{i 3}}{\cos \beta} ; \frac{N_{i 4}}{\sin \beta}\right\} \cos \tilde{\theta}_{f}+\sqrt{2}\left[Q_{f} N_{i 1}^{\prime}-\frac{Q_{f} s_{w}}{c_{w}} N_{i 2}^{\prime}\right] \sin \tilde{\theta}_{f}, \\
& Y_{i 2}^{0\{d ; u\}}:=+\frac{m_{f}}{\sqrt{2} M_{W} s_{w}}\left\{\frac{N_{i 3}}{\cos \beta} ; \frac{N_{i 4}}{\sin \beta}\right\} \sin \tilde{\theta}_{f}+\sqrt{2}\left[Q_{f} N_{i 1}^{\prime}-\frac{Q_{f} s_{w}}{c_{w}} N_{i 2}^{\prime}\right] \cos \tilde{\theta}_{f},
\end{aligned}
$$

with

$$
\begin{aligned}
& N_{i 1}^{\prime}:=N_{i 1} c_{w}+N_{i 2} s_{w}, \\
& N_{i 2}^{\prime}:=N_{i 2} c_{w}-N_{i 1} s_{w} .
\end{aligned}
$$

and the matrices $U_{i j}, V_{i j}$ for the charginos and $N_{i j}$ for the neutralinos, see Appendices A.3, A.4.

The color factor $C_{F}$ is defined in Eq. (C.9), and the factors $g_{H}^{f}$ of the Yukawa couplings can be found in Table B.1.

\begin{tabular}{|c||c|c|c|c|c|}
\hline$g_{H}^{f}$ & $H=$ SM-Higgs & $H=h^{0}$ & $H=H^{0}$ & $H=A^{0}$ & $H=H^{ \pm}$ \\
\hline \hline$f=u$-type & 1 & $\frac{\cos \alpha}{\sin \beta}$ & $\frac{\sin \alpha}{\sin \beta}$ & $\cot \beta$ & $\cot \beta$ \\
\hline$f=d$-type & 1 & $-\frac{\sin \alpha}{\cos \beta}$ & $\frac{\cos \alpha}{\cos \beta}$ & $\tan \beta$ & $\tan \beta$ \\
\hline
\end{tabular}

Table B.1: Yukawa coupling coefficients. 

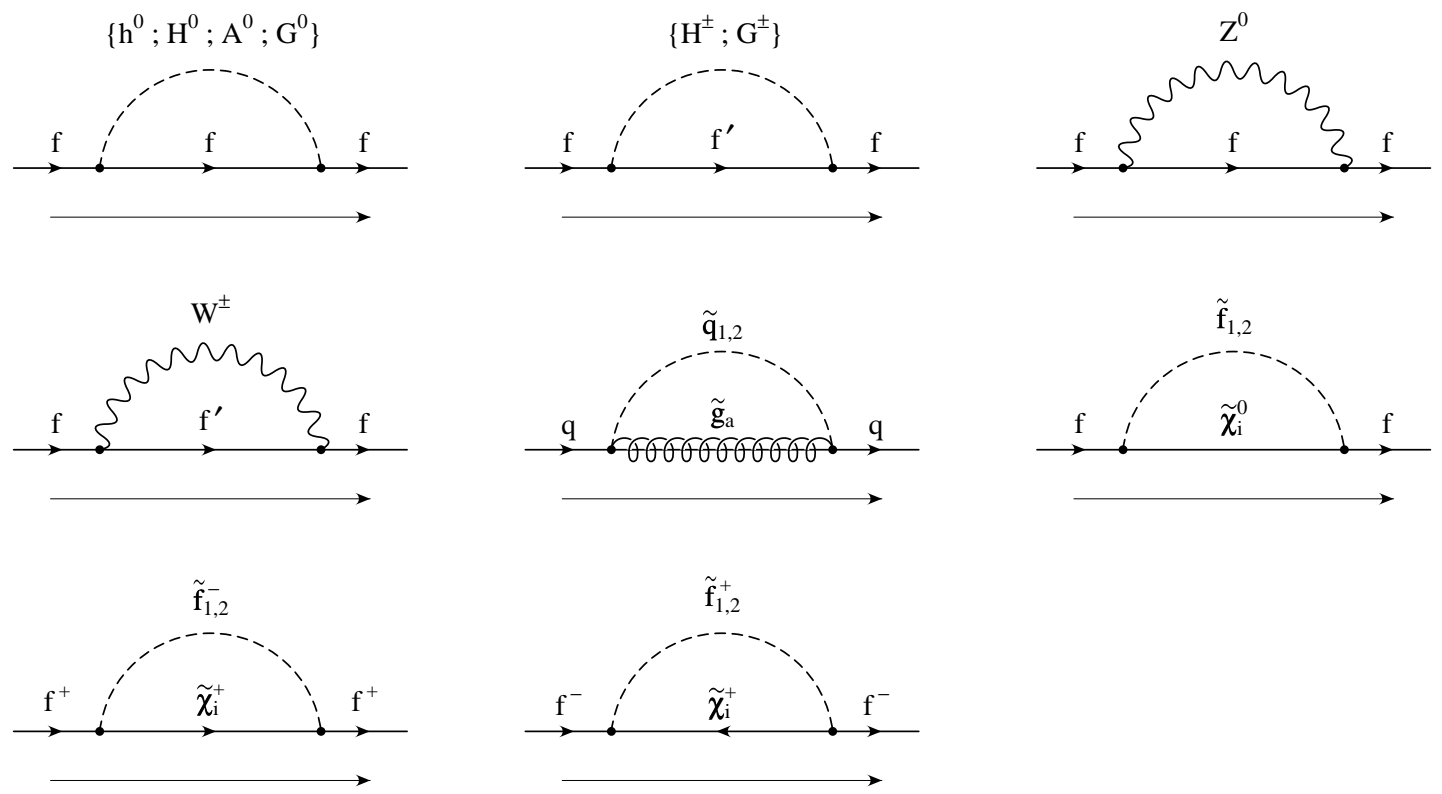

The scalar part $\Sigma_{S}^{f}\left(p^{2}\right)$ :

$$
\begin{aligned}
\Sigma_{S, 2 H D}^{f}\left(p^{2}\right)= & -\frac{\alpha}{4 \pi}\left\{\left(v_{f}^{2}-a_{f}^{2}\right)\left[4 B_{0}\left(p^{2}, m_{f}, M_{Z}\right)-2\right]\right. \\
& +\frac{m_{f^{\prime}}^{2}}{2 s_{w}^{2} M_{W}^{2}}\left[B_{0}\left(p^{2}, m_{f^{\prime}}, M_{W}\right)-B_{0}\left(p^{2}, m_{f^{\prime}}, m_{H^{ \pm}}\right)\right] \\
& +\frac{m_{f}^{2}}{4 s_{w}^{2} M_{W}^{2}}\left[B_{0}\left(p^{2}, m_{f}, M_{Z}\right)-\left(g_{h^{0}}^{f}\right)^{2} B_{0}\left(p^{2}, m_{f}, m_{h^{0}}\right)\right. \\
& \left.\left.-\left(g_{H^{0}}^{f}\right)^{2} B_{0}\left(p^{2}, m_{f}, m_{H^{0}}\right)+\left(g_{A^{0}}^{f}\right)^{2} B_{0}\left(p^{2}, m_{f}, M_{A^{0}}\right)\right]\right\}, \\
\Sigma_{S, g l u i n o}^{f}\left(p^{2}\right)= & -\frac{\alpha_{s}\left(M_{Z}^{2}\right)}{4 \pi} \frac{m_{\tilde{g}}}{m_{f}}\left(2 C_{F} s_{\tilde{\theta}} c_{\tilde{\theta}}\right)\left[B_{0}\left(p^{2}, m_{\tilde{g}}, m_{\tilde{q}_{1}}\right)-B_{0}\left(p^{2}, m_{\tilde{g}}, m_{\tilde{q}_{2}}\right)\right], \\
\Sigma_{S, S U S Y}^{f}\left(p^{2}\right)= & \frac{\alpha}{4 \pi}\left\{\sum_{i=1}^{4} \frac{m_{\tilde{\chi}_{i}^{0}}}{m_{f}}\left[\Re \mathrm{e}\left(X_{i 1}^{0 f} Y_{i 1}^{0 f}\right) B_{0}\left(p^{2}, m_{\tilde{\chi}_{i}^{0}}, m_{\tilde{f}_{1}}\right)+\Re \mathrm{e}\left(X_{i 2}^{0 f} Y_{i 2}^{0 f}\right) B_{0}\left(p^{2}, m_{\tilde{\chi}_{i}^{0}}, m_{\tilde{f}_{2}}\right)\right]\right. \\
& +\sum_{i=1}^{2} \frac{m_{\tilde{\chi}_{i}^{+}}}{m_{f}}\left[\Re \mathrm{e}\left(X_{i 1}^{+f} Y_{i 1}^{+f}\right) B_{0}\left(p^{2}, m_{\tilde{\chi}_{i}^{+}}, m_{\tilde{f}_{1}^{\prime}}\right)\right. \\
& \left.\left.+\Re \mathrm{e}\left(X_{i 2}^{+f} Y_{i 2}^{+f}\right) B_{0}\left(p^{2}, m_{\tilde{\chi}_{i}^{+}}, m_{\tilde{f}_{2}^{\prime}}\right)\right]\right\} \cdot
\end{aligned}
$$


The vector part $\Sigma_{V}^{f}\left(p^{2}\right)$ :

$$
\begin{aligned}
& \Sigma_{V, 2 H D}^{f}\left(p^{2}\right)=-\frac{\alpha}{4 \pi}\left\{\left(v_{f}^{2}+a_{f}^{2}\right)\left[2 B_{1}\left(p^{2}, m_{f}, M_{Z}\right)+1\right]+\frac{1}{4 s_{w}^{2}}\left[2 B_{1}\left(p^{2}, m_{f^{\prime}}, M_{W}\right)+1\right]\right. \\
&+\frac{m_{f}^{2}}{4 s_{w}^{2} M_{W}^{2}}\left[\left(g_{h^{0}}^{f}\right)^{2} B_{1}\left(p^{2}, m_{f}, m_{h^{0}}\right)+\left(g_{H^{0}}^{f}\right)^{2} B_{1}\left(p^{2}, m_{f}, m_{H^{0}}\right)\right. \\
&\left.+\left(g_{A^{0}}^{f}\right)^{2} B_{1}\left(p^{2}, m_{f}, M_{A^{0}}\right)+B_{1}\left(p^{2}, m_{f}, M_{Z}\right)\right] \\
&+\frac{1}{4 s_{w}^{2} M_{W}^{2}}\left[\left(m_{f}^{2}+m_{f^{\prime}}^{2}\right) B_{1}\left(p^{2}, m_{f^{\prime}}, M_{W}\right)\right. \\
&\left.\left.+\left(m_{f}^{2}\left(g_{H^{ \pm}}^{f}\right)^{2}+m_{f^{\prime}}^{2}\left(g_{H^{ \pm}}^{f^{\prime}}\right)^{2}\right) B_{1}\left(p^{2}, m_{f^{\prime}}, m_{H^{ \pm}}\right)\right]\right\} \\
& \Sigma_{V, \text { gluino }}^{f}\left(p^{2}\right)=-\frac{\alpha_{s}\left(M_{Z}^{2}\right)}{4 \pi} C_{F}\left[B_{1}\left(p^{2}, m_{\tilde{g}}, m_{\tilde{q}_{1}}\right)+B_{1}\left(p^{2}, m_{\tilde{g}}, m_{\tilde{q}_{2}}\right)\right] \\
& \Sigma_{V, S U S Y}^{f}\left(p^{2}\right)=-\frac{\alpha}{4 \pi}\left\{\sum _ { i = 1 } ^ { 2 } \left(\frac{1}{2}\left[\left|X_{i 1}^{+f}\right|^{2}+\left|Y_{i 1}^{+f}\right|^{2}\right] B_{1}\left(p^{2}, m_{\tilde{\chi}_{i}^{+}}, m_{\tilde{f}_{1}^{\prime}}\right)\right.\right. \\
&\left.+\frac{1}{2}\left[\left|X_{i 2}^{+f}\right|^{2}+\left|Y_{i 2}^{+f}\right|^{2}\right] B_{1}\left(p^{2}, m_{\tilde{\chi}_{i}^{+}}, m_{\tilde{f}_{2}^{\prime}}\right)\right) \\
&+\sum_{i=1}^{4}\left(\frac{1}{2}\left[\left|X_{i 1}^{0 f}\right|^{2}+\left|Y_{i 1}^{0 f}\right|^{2}\right] B_{1}\left(p^{2}, m_{\tilde{\chi}_{i}^{0}}, m_{\tilde{f}_{1}}\right)\right. \\
&\left.\left.+\frac{1}{2}\left[\left|X_{i 2}^{0 f}\right|^{2}+\left|Y_{i 2}^{0 f}\right|^{2}\right] B_{1}\left(p^{2}, m_{\tilde{\chi}_{i}^{0}}, m_{\tilde{f}_{2}}\right)\right)\right\} . \\
&(\mathrm{B}
\end{aligned}
$$

The axial vector part $\Sigma_{A}^{f}\left(p^{2}\right)$ :

$$
\begin{aligned}
\Sigma_{A, 2 H D}^{f}\left(p^{2}\right)= & -\frac{\alpha}{4 \pi}\left\{-2 v_{f} a_{f}\left[2 B_{1}\left(p^{2}, m_{f}, M_{Z}\right)+1\right]-\frac{1}{4 s_{w}^{2}}\left[2 B_{1}\left(p^{2}, m_{f^{\prime}}, M_{W}\right)+1\right]\right. \\
& +\frac{1}{4 s_{w}^{2} M_{W}^{2}}\left[\left(m_{f}^{2}-m_{f^{\prime}}^{2}\right) B_{1}\left(p^{2}, m_{f^{\prime}}, M_{W}\right)\right. \\
& \left.\left.+\left(m_{f}^{2}\left(g_{H^{ \pm}}^{f}\right)^{2}-m_{f^{\prime}}^{2}\left(g_{H^{ \pm}}^{f^{\prime}}\right)^{2}\right) B_{1}\left(p^{2}, m_{f^{\prime}}, m_{H^{ \pm}}\right)\right]\right\} \\
\Sigma_{A, \text { gluino }}^{f}\left(p^{2}\right)= & -\frac{\alpha_{s}\left(M_{Z}^{2}\right)}{4 \pi} C_{F}\left\{c_{\tilde{\theta}}^{2}\left[B_{1}\left(p^{2}, m_{\tilde{g}}, m_{\tilde{q}_{2}}\right)-B_{1}\left(p^{2}, m_{\tilde{g}}, m_{\tilde{q}_{1}}\right)\right]\right. \\
& \left.+s_{\tilde{\theta}}^{2}\left[B_{1}\left(p^{2}, m_{\tilde{g}}, m_{\tilde{q}_{1}}\right)-B_{1}\left(p^{2}, m_{\tilde{g}}, m_{\tilde{q}_{2}}\right)\right]\right\} \\
\Sigma_{A, S U S Y}^{f}\left(p^{2}\right)= & \frac{\alpha}{4 \pi}\left\{\sum _ { i = 1 } ^ { 2 } \left(\frac{1}{2}\left[\left|X_{i 1}^{+f}\right|^{2}-\left|Y_{i 1}^{+f}\right|^{2}\right] B_{1}\left(p^{2}, m_{\tilde{\chi}_{i}^{+}}, m_{\tilde{f}_{1}^{\prime}}\right)\right.\right.
\end{aligned}
$$




$$
\begin{aligned}
& \left.+\frac{1}{2}\left[\left|X_{i 2}^{+f}\right|^{2}-\left|Y_{i 2}^{+f}\right|^{2}\right] B_{1}\left(p^{2}, m_{\tilde{\chi}_{i}^{+}}, m_{\tilde{f}_{2}^{\prime}}\right)\right) \\
& +\sum_{i=1}^{4}\left(\frac{1}{2}\left[\left|X_{i 1}^{0 f}\right|^{2}-\left|Y_{i 1}^{0 f}\right|^{2}\right] B_{1}\left(p^{2}, m_{\tilde{\chi}_{i}^{0}}, m_{\tilde{f}_{1}}\right)\right. \\
& \left.\left.+\frac{1}{2}\left[\left|X_{i 2}^{0 f}\right|^{2}-\left|Y_{i 2}^{0 f}\right|^{2}\right] B_{1}\left(p^{2}, m_{\tilde{\chi}_{i}^{0}}, m_{\tilde{f}_{2}}\right)\right)\right\} .
\end{aligned}
$$

\section{Vertex corrections}

This appendix contains all 3-point vertex diagrams for $\gamma f \bar{f}$ and $Z f \bar{f}$ within the MSSM at the 1-loop level (without virtual photons and gluons). The diagrams with Higgs bosons are negligible for $f=e$.

In addition to Appendix $\mathrm{B}$ we define the following shorthand notations for the couplings:

$$
\begin{aligned}
& g_{Z, R}=-\frac{s_{w}}{c_{w}} Q_{f} \quad, \quad g_{Z, L}=\frac{I_{3}^{f}-s_{w}^{2} Q_{f}}{s_{w} c_{w}} \quad, \quad g_{C}=\frac{1}{\sqrt{2} s_{w}} \frac{m_{f}}{M_{W}} \\
& g_{Z, R}^{\prime}=-\frac{s_{w}}{c_{w}} Q_{f^{\prime}} \quad, \quad g_{Z, L}^{\prime}=\frac{I_{3}^{f^{\prime}}-s_{w}^{2} Q_{f^{\prime}}}{s_{w} c_{w}} \quad, \quad g_{C}^{\prime}=\frac{1}{\sqrt{2} s_{w}} \frac{m_{f^{\prime}}}{M_{W}} \\
& g_{N}=-\frac{1}{2 s_{w}} \frac{m_{f}}{M_{W}} .
\end{aligned}
$$

3-point functions $C$ are discussed in Refs. [27, 29, 30], but we use the convention of Refs. [13], 15]. The arguments for the $C$-functions are $C\left(p_{1}, p_{2}, m_{1}, m_{2}, m_{3}\right)$. For the electron vertex corrections we have $p_{1}=p, p_{2}=-p-\bar{p}, p_{3}=\bar{p}$, and for the outgoing fermions we have $p_{1}=-\bar{k}, p_{2}=k+\bar{k}$, $p_{3}=-k$.

The conventions on momenta and internal masses are illustrated in Figure C.1 (the arrows within the lines denote the charge flow and long, thin arrows denote the fermion flow if necessary).

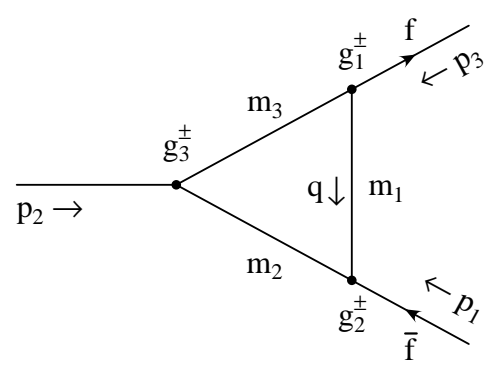

Figure C.1: The vertex topology.

Next we give the generic expressions for the form factors of each class of vertex diagrams. The contributing particles and their couplings are listed in the attached tables. 


\section{C.1 Class-I diagram}

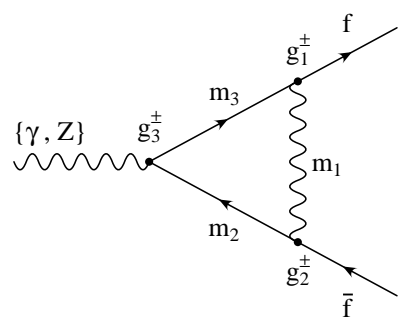

$$
\begin{aligned}
A_{I}^{ \pm}= & {\left[4 C_{24}-2+2 m_{f}^{2}\left(2 C_{0}+3 C_{11}+C_{21}\right)-2 s\left(C_{0}+C_{11}+C_{23}-C_{22}\right)\right] g_{1}^{ \pm} g_{2}^{ \pm} g_{3}^{ \pm} } \\
& +2 m_{f}^{2}\left(C_{0}+C_{11}\right) g_{1}^{\mp} g_{2}^{\mp} g_{3}^{\mp}-2 m_{\hat{f}}^{2} C_{0} g_{1}^{ \pm} g_{2}^{ \pm} g_{3}^{\mp}, \\
B_{I}^{ \pm}= & 2\left\{m_{\hat{f}}\left(C_{0}+C_{11}\right) g_{1}^{\mp} g_{2}^{ \pm}\left(g_{3}^{ \pm}+g_{3}^{\mp}\right)-m_{f}\left[\left(C_{0}+C_{11}+C_{12}+C_{23}\right) g_{1}^{ \pm} g_{2}^{ \pm} g_{3}^{ \pm}\right.\right. \\
& \left.\left.+\left(C_{0}+2 C_{11}-C_{12}+C_{21}-C_{23}\right) g_{1}^{\mp} g_{2}^{\mp} g_{3}^{\mp}\right]\right\} .
\end{aligned}
$$

\begin{tabular}{|c||c|c|c|c|c|c|}
\hline \multicolumn{7}{|c|}{ Masses and coupling constants for the $\gamma f \bar{f}$ vertex I } \\
\hline$m_{1} m_{2} m_{3}$ & $g_{1}^{+}$ & $g_{1}^{-}$ & $g_{2}^{+}$ & $g_{2}^{-}$ & $g_{3}^{+}$ & $g_{3}^{-}$ \\
\hline \hline $\mathrm{Z} \quad \mathrm{f}$ & $g_{Z, R}$ & $g_{Z, L}$ & $g_{Z, R}$ & $g_{Z, L}$ & $-Q_{f}$ & $-Q_{f}$ \\
\hline $\mathrm{W} \quad \mathrm{f}^{\prime} \mathrm{f}^{\prime}$ & 0 & $\frac{1}{\sqrt{2} s_{w}}$ & 0 & $\frac{1}{\sqrt{2} s_{w}}$ & $-Q_{f^{\prime}}$ & $-Q_{f^{\prime}}$ \\
\hline
\end{tabular}

Masses and coupling constants for the $Z f \bar{f}$ vertex I

\begin{tabular}{|c||c|c|c|c|c|c|}
\hline$m_{1} m_{2} m_{3}$ & $g_{1}^{+}$ & $g_{1}^{-}$ & $g_{2}^{+}$ & $g_{2}^{-}$ & $g_{3}^{+}$ & $g_{3}^{-}$ \\
\hline \hline $\mathrm{Z} \quad \mathrm{f}$ & $g_{Z, R}$ & $g_{Z, L}$ & $g_{Z, R}$ & $g_{Z, L}$ & $g_{Z, R}$ & $g_{Z, L}$ \\
\hline $\mathrm{W} \mathrm{f}^{\prime} \mathrm{f}^{\prime}$ & 0 & $\frac{1}{\sqrt{2} s_{w}}$ & 0 & $\frac{1}{\sqrt{2} s_{w}}$ & $g_{Z, R}^{\prime}$ & $g_{Z, L}^{\prime}$ \\
\hline
\end{tabular}




\section{C.2 Class-II diagram}

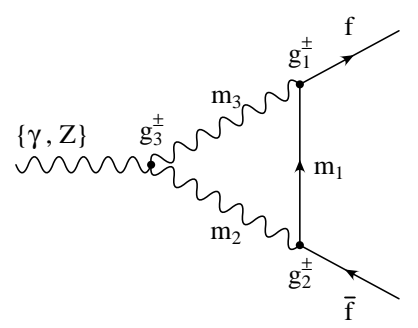

$$
\begin{aligned}
& A_{I I}^{+}=3 m_{f}^{2} C_{11} g_{1}^{-} g_{2}^{-} g_{3}^{-} \\
& A_{I I}^{-}=\left[12 C_{24}-2+2 m_{f}^{2} C_{21}-2 s\left(C_{23}-C_{22}\right)+\left(5 m_{f}^{2}-2 s\right) C_{11}\right] g_{1}^{-} g_{2}^{-} g_{3}^{-}, \\
& B_{I I}^{+}=m_{f}\left(2 C_{21}-2 C_{23}-C_{12}\right) g_{1}^{-} g_{2}^{-} g_{3}^{-}, \\
& B_{I I}^{-}=m_{f}\left(2 C_{23}-C_{11}+C_{12}\right) g_{1}^{-} g_{2}^{-} g_{3}^{-} .
\end{aligned}
$$

\begin{tabular}{|c||c|c|c|c|c|}
\hline \multicolumn{6}{|c|}{ Masses and coupling constants for the $\gamma f \bar{f}$ vertex II } \\
\hline$m_{1} m_{2} m_{3}$ & $g_{1}^{+}$ & $g_{1}^{-}$ & $g_{2}^{+}$ & $g_{2}^{-}$ & $g_{3}^{+}=g_{3}^{-}$ \\
\hline \hline $\mathrm{f}^{\prime} \mathrm{W} \mathrm{W}$ & 0 & $\frac{1}{\sqrt{2} s_{w}}$ & 0 & $\frac{1}{\sqrt{2} s_{w}}$ & $-2 I_{3}^{f}$ \\
\hline
\end{tabular}

\begin{tabular}{|c||c|c|c|c|c|}
\hline \multicolumn{6}{|c|}{ Masses and coupling constants for the $Z f \bar{f}$ vertex II } \\
\hline$m_{1} m_{2} m_{3}$ & $g_{1}^{+}$ & $g_{1}^{-}$ & $g_{2}^{+}$ & $g_{2}^{-}$ & $g_{3}^{+}=g_{3}^{-}$ \\
\hline \hline $\mathrm{f}^{\prime} \mathrm{W} \mathrm{W}$ & 0 & $\frac{1}{\sqrt{2} s_{w}}$ & 0 & $\frac{1}{\sqrt{2} s_{w}}$ & $2 I_{3}^{f} \frac{c_{w}}{s_{w}}$ \\
\hline
\end{tabular}




\section{C.3 Class-III diagram}
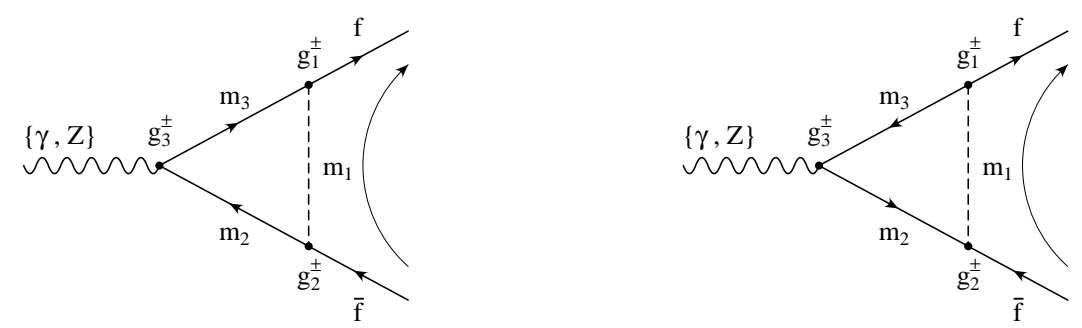

In class-III, the dashed line indicates either a Higgs particle or a sfermion.

$$
\begin{aligned}
A_{V}^{ \pm}= & {\left[2 C_{24}-\frac{1}{2}+m_{f}^{2}\left(C_{11}+C_{21}\right)-s\left(C_{23}-C_{22}\right)\right] g_{1}^{\mp} g_{2}^{ \pm} g_{3}^{\mp} } \\
& -m_{2} m_{3} C_{0} g_{1}^{\mp} g_{2}^{ \pm} g_{3}^{ \pm}-m_{f}^{2}\left(C_{0}+C_{11}\right) g_{1}^{ \pm} g_{2}^{\mp} g_{3}^{ \pm} \\
& -m_{f} m_{2}\left[\left(C_{0}+C_{12}\right) g_{1}^{ \pm} g_{2}^{ \pm} g_{3}^{ \pm}-\left(C_{11}-C_{12}\right) g_{1}^{\mp} g_{2}^{\mp} g_{3}^{\mp}\right], \\
& -m_{f} m_{3}\left[\left(C_{0}+C_{11}-C_{12}\right) g_{1}^{\mp} g_{2}^{\mp} g_{3}^{ \pm}-C_{12} g_{1}^{ \pm} g_{2}^{ \pm} g_{3}^{\mp}\right] \\
B_{V}^{ \pm}= & -\left\{\left[m_{2}\left(C_{11}-C_{12}\right) g_{3}^{ \pm}+m_{3} C_{12} g_{3}^{\mp}\right] g_{1}^{ \pm} g_{2}^{ \pm}\right. \\
& \left.+m_{f}\left[\left(C_{11}-C_{12}+C_{21}-C_{23}\right) g_{1}^{ \pm} g_{2}^{\mp} g_{3}^{ \pm}+\left(C_{12}+C_{23}\right) g_{1}^{\mp} g_{2}^{ \pm} g_{3}^{\mp}\right]\right\} .
\end{aligned}
$$

The tables contain the extra notations

$$
\begin{aligned}
& O_{a}^{f}=\left\{\begin{array}{rl}
O_{j i}^{\prime R} & \text { for } f=u \text {-type } \\
-O_{i j}^{\prime L} & \text { for } f=d \text {-type }
\end{array},\right. \\
& O_{b}^{f}=\left\{\begin{aligned}
O_{j i}^{\prime L} & \text { for } f=u \text {-type } \\
-O_{i j}^{\prime R} & \text { for } f=d \text {-type }
\end{aligned}\right.
\end{aligned}
$$

$O_{i j}^{L, R}$ and $O_{i j}^{\prime \prime L, R}$ are defined in Eq. (B.8) and $X_{k l}^{0 f}, X_{k l}^{+f}, Y_{k l}^{0 f}$ and $Y_{k l}^{+f}$ in Eqs. (B.15). 


\begin{tabular}{|c|c|c|c|c|c|c|}
\hline \multicolumn{7}{|c|}{ Masses and coupling constants for the $\gamma f \bar{f}$ vertex III } \\
\hline$m_{1} m_{2} m_{3}$ & $g_{1}^{+}$ & $g_{1}^{-}$ & $g_{2}^{+}$ & $g_{2}^{-}$ & $g_{3}^{+}$ & $g_{3}^{-}$ \\
\hline $\mathrm{H}^{0} \quad \mathrm{f} \quad \mathrm{f}$ & $g_{N} g_{H^{0}}^{f}$ & $g_{N} g_{H^{0}}^{f}$ & $g_{N} g_{H^{0}}^{f}$ & $g_{N} g_{H^{0}}^{f}$ & $-Q_{f}$ & $-Q_{f}$ \\
\hline $\mathrm{h}^{0} \mathrm{f} \quad \mathrm{f}$ & $g_{N} g_{h^{0}}^{f}$ & $g_{N} g_{h^{0}}^{f}$ & $g_{N} g_{h^{0}}^{f}$ & $g_{N} g_{h^{0}}^{f}$ & $-Q_{f}$ & $-Q_{f}$ \\
\hline $\mathrm{A}^{0} \mathrm{f} \quad \mathrm{f}$ & $-i g_{N} g_{A^{0}}^{f}$ & $i g_{N} g_{A^{0}}^{f}$ & $-i g_{N} g_{A^{0}}^{f}$ & $i g_{N} g_{A^{0}}^{f}$ & $-Q_{f}$ & $-Q_{f}$ \\
\hline $\mathrm{G}^{0} \mathrm{f} \mathrm{f}$ & $-i g_{N} 2 I_{3}^{f}$ & $i g_{N} 2 I_{3}^{f}$ & $-i g_{N} 2 I_{3}^{f}$ & $i g_{N} 2 I_{3}^{f}$ & $-Q_{f}$ & $-Q_{f}$ \\
\hline $\mathrm{H}^{ \pm} \quad \mathrm{f}^{\prime} \quad \mathrm{f}^{\prime}$ & $g_{C}^{\prime} g_{H^{ \pm}}^{f^{\prime}}$ & $g_{C} g_{H^{ \pm}}^{f}$ & $g_{C} g_{H^{ \pm}}^{f}$ & $g_{C}^{\prime} g_{H^{ \pm}}^{f^{\prime}}$ & $-Q_{f^{\prime}}$ & $-Q_{f^{\prime}}$ \\
\hline $\mathrm{G}^{ \pm} \quad \mathrm{f}^{\prime} \quad \mathrm{f}^{\prime}$ & $g_{C}^{\prime} 2 I_{3}^{f^{\prime}}$ & $g_{C} 2 I_{3}^{f}$ & $g_{C} 2 I_{3}^{f}$ & $g_{C}^{\prime} 2 I_{3}^{f^{\prime}}$ & $-Q_{f^{\prime}}$ & $-Q_{f^{\prime}}$ \\
\hline$\tilde{\mathrm{f}}_{1}^{\prime} \tilde{\chi}_{\mathrm{i}}^{+} \tilde{\chi}_{\mathrm{i}}^{+}$ & $X_{i 1}^{+f}$ & $\left(Y_{i 1}^{+f}\right)^{*}$ & $Y_{i 1}^{+f}$ & $\left(X_{i 1}^{+f}\right)^{*}$ & $-2 I_{3}^{f}$ & $-2 I_{3}^{f}$ \\
\hline$\tilde{\mathrm{f}}_{2}^{\prime} \tilde{\chi}_{\mathrm{i}}^{+} \tilde{\chi}_{\mathrm{i}}^{+}$ & $X_{i 2}^{+f}$ & $\left(Y_{i 2}^{+f}\right)^{*}$ & $Y_{i 2}^{+f}$ & $\left(X_{i 2}^{+f}\right)^{*}$ & $-2 I_{3}^{f}$ & $-2 I_{3}^{f}$ \\
\hline
\end{tabular}

\begin{tabular}{|c|c|c|c|c|c|c|}
\hline \multicolumn{7}{|c|}{ Masses and coupling constants for the $Z f \bar{f}$ vertex III } \\
\hline$m_{1} m_{2} m_{3}$ & $g_{1}^{+}$ & $g_{1}^{-}$ & $g_{2}^{+}$ & $g_{2}^{-}$ & $g_{3}^{+}$ & $g_{3}^{-}$ \\
\hline $\mathrm{H}^{0} \quad \mathrm{f} \quad \mathrm{f}$ & $g_{N} g_{H^{0}}^{f}$ & $g_{N} g_{H^{0}}^{f}$ & $g_{N} g_{H^{0}}^{f}$ & $g_{N} g_{H^{0}}^{f}$ & $g_{Z, R}$ & $g_{Z, L}$ \\
\hline $\mathrm{h}^{0} \mathrm{f} \quad \mathrm{f}$ & $g_{N} g_{h^{0}}^{f}$ & $g_{N} g_{h^{0}}^{f}$ & $g_{N} g_{h^{0}}^{f}$ & $g_{N} g_{h^{0}}^{f}$ & $g_{Z, R}$ & $g_{Z, L}$ \\
\hline $\mathrm{A}^{0} \mathrm{f} \quad \mathrm{f}$ & $-i g_{N} g_{A^{0}}^{f}$ & $i g_{N} g_{A^{0}}^{f}$ & $-i g_{N} g_{A^{0}}^{f}$ & $i g_{N} g_{A^{0}}^{f}$ & $g_{Z, R}$ & $g_{Z, L}$ \\
\hline $\mathrm{G}^{0} \mathrm{f} \quad \mathrm{f}$ & $-i g_{N} 2 I_{3}^{f}$ & $i g_{N} 2 I_{3}^{f}$ & $-i g_{N} 2 I_{3}^{f}$ & $i g_{N} 2 I_{3}^{f}$ & $g_{Z, R}$ & $g_{Z, L}$ \\
\hline $\mathrm{H}^{ \pm} \mathrm{f}^{\prime} \quad \mathrm{f}^{\prime}$ & $g_{C}^{\prime} g_{H^{ \pm}}^{f^{\prime}}$ & $g_{C} g_{H^{ \pm}}^{f}$ & $g_{C} g_{H^{ \pm}}^{f}$ & $g_{C}^{\prime} g_{H^{ \pm}}^{f^{\prime}}$ & $g_{Z, R}^{\prime}$ & $g_{Z, L}^{\prime}$ \\
\hline $\mathrm{G}^{ \pm} \mathrm{f}^{\prime} \quad \mathrm{f}^{\prime}$ & $g_{C}^{\prime} 2 I_{3}^{f^{\prime}}$ & $g_{C} 2 I_{3}^{f}$ & $g_{C} 2 I_{3}^{f}$ & $g_{C}^{\prime} 2 I_{3}^{f^{\prime}}$ & $g_{Z, R}^{\prime}$ & $g_{Z, L}^{\prime}$ \\
\hline$\tilde{\mathrm{f}}_{1} \quad \tilde{\chi}_{\mathrm{i}}^{0} \quad \tilde{\chi}_{\mathrm{j}}^{0}$ & $X_{j 1}^{0 f}$ & $\left(Y_{j 1}^{0 f}\right)^{*}$ & $Y_{i 1}^{0 f}$ & $\left(X_{i 1}^{0 f}\right)^{*}$ & $-\frac{1}{s_{w} c_{y}} O_{j i}^{\prime \prime R}$ & $-\frac{1}{s_{\psi} c_{\psi}} O_{j i}^{\prime \prime L}$ \\
\hline$\tilde{\mathrm{f}}_{2} \quad \tilde{\chi}_{\mathrm{i}}^{0} \quad \tilde{\chi}_{\mathrm{j}}^{0}$ & $X_{j 2}^{0 f}$ & $\left(Y_{j 2}^{0 f}\right)^{*}$ & $Y_{i 2}^{0 f}$ & $\left(X_{i 2}^{0 f}\right)^{*}$ & $-\frac{1}{s_{w} c_{\psi}} O_{j i}^{\prime \prime R}$ & $-\frac{1}{s_{w} c_{w}} O_{j i}^{\prime \prime L}$ \\
\hline$\tilde{\mathrm{f}}_{1}^{\prime} \quad \tilde{\chi}_{\mathrm{i}}^{+} \tilde{\chi}_{\mathrm{j}}^{+}$ & $X_{j 1}^{+f}$ & $\left(Y_{j 1}^{+f}\right)^{*}$ & $Y_{i 1}^{+f}$ & $\left(X_{i 1}^{+f}\right)^{*}$ & $-\frac{1}{s_{\psi} c_{\psi}} O_{a}^{f}$ & $-\frac{1}{s_{w} c_{w}} O_{b}^{f}$ \\
\hline $\begin{array}{lll}\tilde{\mathrm{f}}_{2}^{\prime} & \tilde{\chi}_{\mathrm{i}}^{+} & \tilde{\chi}_{\mathrm{j}}^{+}\end{array}$ & $X_{j 2}^{+f}$ & $\left(Y_{j 2}^{+f}\right)^{*}$ & $Y_{i 2}^{+f}$ & $\left(X_{i 2}^{+f}\right)^{*}$ & $-\frac{1}{s_{w} c_{w}} O_{a}^{f}$ & $-\frac{1}{s_{w} c_{w}} O_{b}^{f}$ \\
\hline
\end{tabular}




\section{C.4 Class-IV diagram}
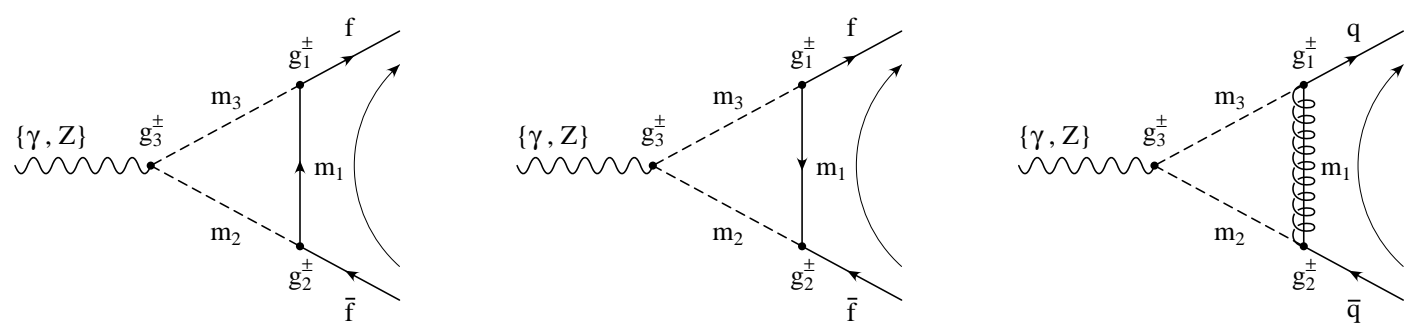

The signature for the dashed lines is as in class-V. In addition there is the gluino diagram.

$$
\begin{aligned}
A_{V I}^{ \pm}= & 2 C_{24} g_{1}^{\mp} g_{2}^{ \pm} g_{3}^{ \pm}, \\
B_{V I}^{ \pm}= & g_{3}^{ \pm}\left\{m_{f}\left[\left(C_{12}+C_{23}\right) g_{1}^{\mp} g_{2}^{ \pm}+\left(C_{11}-C_{12}+C_{21}-C_{23}\right) g_{1}^{ \pm} g_{2}^{\mp}\right]\right. \\
& \left.-m_{1}\left(C_{0}+C_{11}\right) g_{1}^{ \pm} g_{2}^{ \pm}\right\} .
\end{aligned}
$$

The following shorthand notations are used:

$$
\begin{aligned}
g_{Z, 1} & =\frac{I_{3}^{f} c_{\tilde{\theta}}^{2}-Q_{f} s_{w}^{2}}{s_{w} c_{w}}, \\
g_{Z, 2} & =\frac{I_{3}^{f} s_{\tilde{\theta}}^{2}-Q_{f} s_{w}^{2}}{s_{w} c_{w}}, \\
g_{Z} & =-\frac{I_{3}^{f} s_{\tilde{\theta}} c_{\tilde{\theta}}}{s_{w} c_{w}}, \\
g_{Z, 1}^{\prime} & =\frac{I_{3}^{f^{\prime}} c_{\tilde{\theta}^{\prime}}^{2}-Q_{f^{\prime}} s_{w}^{2}}{s_{w} c_{w}}, \\
g_{Z, 2}^{\prime} & =\frac{I_{3}^{f^{\prime}} s_{\tilde{\theta}^{\prime}}^{2}-Q_{f^{\prime}} s_{w}^{2}}{s_{w} c_{w}}, \\
g_{Z}^{\prime} & =-\frac{I_{3}^{f^{\prime}} s_{\tilde{\theta}^{\prime}} c_{\tilde{\theta}}^{\prime}}{s_{w} c_{w}},
\end{aligned}
$$

with $c_{\tilde{\theta}}:=\cos \tilde{\theta}_{f}, s_{\tilde{\theta}}:=\sin \tilde{\theta}_{f}$ and $c_{\tilde{\theta}^{\prime}}:=\cos \tilde{\theta}_{f^{\prime}}, s_{\tilde{\theta}^{\prime}}:=\sin \tilde{\theta}_{f^{\prime}}$. 


\begin{tabular}{|c|c|c|c|c|c|}
\hline \multicolumn{6}{|c|}{ Masses and coupling constants for the $\gamma f \bar{f}$ vertex IV } \\
\hline$m_{1} m_{2} m_{3}$ & $g_{1}^{+}$ & $g_{1}^{-}$ & $g_{2}^{+}$ & $g_{2}^{-}$ & $g_{3}^{+}=g_{3}^{-}$ \\
\hline $\mathrm{f}^{\prime} \mathrm{H}^{ \pm} \mathrm{H}^{ \pm}$ & $g_{C}^{\prime} g_{H^{ \pm}}^{f^{\prime}}$ & $g_{C} g_{H^{ \pm}}^{f}$ & $g_{C} g_{H^{ \pm}}^{f}$ & $g_{C}^{\prime} g_{H^{ \pm}}^{f^{\prime}}$ & $-2 I_{3}^{f}$ \\
\hline $\mathrm{f}^{\prime} \mathrm{G}^{ \pm} \mathrm{G}^{ \pm}$ & $g_{C}^{\prime} 2 I_{3}^{f^{\prime}}$ & $g_{C} 2 I_{3}^{f}$ & $g_{C} 2 I_{3}^{f}$ & $g_{C}^{\prime} 2 I_{3}^{f^{\prime}}$ & $-2 I_{3}^{f}$ \\
\hline $\begin{array}{lll}\tilde{\chi}_{\mathrm{i}}^{0} & \tilde{\mathrm{f}}_{1} & \tilde{\mathrm{f}}_{1}\end{array}$ & $X_{i 1}^{0 f}$ & $\left(Y_{i 1}^{0 f}\right)^{*}$ & $Y_{i 1}^{0 f}$ & $\left(X_{i 1}^{0 f}\right)^{*}$ & $-Q_{f}$ \\
\hline $\begin{array}{lll}\tilde{\chi}_{\mathrm{i}}^{0} & \tilde{\mathrm{f}}_{2} & \tilde{\mathrm{f}}_{2}\end{array}$ & $X_{i 2}^{0 f}$ & $\left(Y_{i 2}^{0 f}\right)^{*}$ & $Y_{i 2}^{0 f}$ & $\left(X_{i 2}^{0 f}\right)^{*}$ & $-Q_{f}$ \\
\hline$\tilde{\chi}_{\mathrm{i}}^{+} \quad \tilde{\mathrm{f}}_{1}^{\prime} \quad \tilde{\mathrm{f}}_{1}^{\prime}$ & $X_{i 1}^{+f}$ & $\left(Y_{i 1}^{+f}\right)^{*}$ & $Y_{i 1}^{+f}$ & $\left(X_{i 1}^{+f}\right)^{*}$ & $-Q_{f^{\prime}}$ \\
\hline$\tilde{\chi}_{\mathrm{i}}^{+} \tilde{\mathrm{f}}_{2}^{\prime} \tilde{\mathrm{f}}_{2}^{\prime}$ & $X_{i 2}^{+f}$ & $\left(Y_{i 2}^{+f}\right)^{*}$ & $Y_{i 2}^{+f}$ & $\left(X_{i 2}^{+f}\right)^{*}$ & $-Q_{f^{\prime}}$ \\
\hline $\begin{array}{lll}\tilde{g}_{\mathrm{a}} & \tilde{\mathrm{q}}_{1} & \tilde{\mathrm{q}}_{1}\end{array}$ & $-\sqrt{2} \frac{g_{3}}{e} c_{\tilde{\theta}} T_{j k}^{a}$ & $\sqrt{2} \frac{g_{3}}{e} s_{\tilde{\theta}} T_{j k}^{a}$ & $\sqrt{2} \frac{g_{3}}{e} s_{\tilde{\theta}} T_{i j}^{a}$ & $-\sqrt{2} \frac{g_{3}}{e} c_{\tilde{\theta}} T_{i j}^{a}$ & $-Q_{q}$ \\
\hline $\begin{array}{lll}\tilde{\mathrm{g}}_{\mathrm{a}} & \tilde{\mathrm{q}}_{2} & \tilde{\mathrm{q}}_{2}\end{array}$ & $\sqrt{2} \frac{g_{3}}{e} s_{\tilde{\theta}} T_{j k}^{a}$ & $\sqrt{2} \frac{g_{3}}{e} c_{\tilde{\theta}} T_{j k}^{a}$ & $\sqrt{2} \frac{g_{3}}{e} c_{\tilde{\theta}} T_{i j}^{a}$ & $\sqrt{2} \frac{g_{3}}{e} s_{\tilde{\theta}} T_{i j}^{a}$ & $-Q_{q}$ \\
\hline
\end{tabular}

The $T_{i j}^{a}(a=1,2, \ldots, 8 ; i, j=1,2,3)$ are the matrix elements of the $S U(3)_{C}$ generators, with the property

$$
\sum_{a, j} T_{i j}^{a} T_{j k}^{a}=\frac{1}{4} \sum_{a, j} \lambda_{i j}^{a} \lambda_{j k}^{a}=\frac{1}{4} \sum_{j}\left(-\frac{2}{3} \delta_{i j} \delta_{j k}+2 \delta_{i k} \delta_{j j}\right) \stackrel{i=k}{=} \frac{4}{3}=: C_{F}
$$

$X_{k l}^{0 f}, X_{k l}^{+f}, Y_{k l}^{0 f}$ and $Y_{k l}^{+f}$ are defined in Eq. (B.15). The factor 1/e in the gluino couplings only appears because of the definition of the matrix elements (2.24) and (2.25). 


\begin{tabular}{|c|c|c|c|c|c|}
\hline \multicolumn{6}{|c|}{ Masses and coupling constants for the $Z f \bar{f}$ vertex IV } \\
\hline$m_{1} m_{2} m_{3}$ & $g_{1}^{+}$ & $g_{1}^{-}$ & $g_{2}^{+}$ & $g_{2}^{-}$ & $g_{3}^{+}=g_{3}^{-}$ \\
\hline f $\mathrm{H}^{0} \mathrm{~A}^{0}$ & $-i g_{N} g_{A^{0}}^{f}$ & $i g_{N} g_{A^{0}}^{f}$ & $g_{N} g_{H^{0}}^{f}$ & $g_{N} g_{H^{0}}^{f}$ & $-i \frac{\sin (\beta-\alpha)}{2 s_{w} c_{w}}$ \\
\hline f $\mathrm{H}^{0} \mathrm{G}^{0}$ & $-i g_{N} 2 I_{3}^{f}$ & $i g_{N} 2 I_{3}^{f}$ & $g_{N} g_{H^{0}}^{f}$ & $g_{N} g_{H^{0}}^{f}$ & $i \frac{\cos (\beta-\alpha)}{2 s_{w} c_{w}}$ \\
\hline $\mathrm{f} \quad \mathrm{h}^{0} \mathrm{~A}^{0}$ & $-i g_{N} g_{A^{0}}^{f}$ & $i g_{N} g_{A^{0}}^{f}$ & $g_{N} g_{h^{0}}^{f}$ & $g_{N} g_{h^{0}}^{f}$ & $i \frac{\cos (\beta-\alpha)}{2 s_{w} c_{w}}$ \\
\hline f $h^{0} G^{0}$ & $-i g_{N} 2 I_{3}^{f}$ & $i g_{N} 2 I_{3}^{f}$ & $g_{N} g_{h^{0}}^{f}$ & $g_{N} g_{h^{0}}^{f}$ & $i \frac{\sin (\beta-\alpha)}{2 s_{w} c_{w}}$ \\
\hline f $\quad \mathrm{A}^{0} \mathrm{H}^{0}$ & $g_{N} g_{H^{0}}^{f}$ & $g_{N} g_{H^{0}}^{f}$ & $-i g_{N} g_{A^{0}}^{f}$ & $i g_{N} g_{A^{0}}^{f}$ & $i \frac{\sin (\beta-\alpha)}{2 s_{w} c_{w}}$ \\
\hline $\mathrm{f} \quad \mathrm{G}^{0} \mathrm{H}^{0}$ & $g_{N} g_{H^{0}}^{f}$ & $g_{N} g_{H^{0}}^{f}$ & $-i g_{N} 2 I_{3}^{f}$ & $i g_{N} 2 I_{3}^{f}$ & $-i \frac{\cos (\beta-\alpha)}{2 s_{w} c_{w}}$ \\
\hline f $A^{0} h^{0}$ & $g_{N} g_{h^{0}}^{f}$ & $g_{N} g_{h^{0}}^{f}$ & $-i g_{N} g_{A^{0}}^{f}$ & $i g_{N} g_{A^{0}}^{f}$ & $-i \frac{\cos (\beta-\alpha)}{2 s_{w} c_{w}}$ \\
\hline $\mathrm{f} \quad \mathrm{G}^{0} \mathrm{~h}^{0}$ & $g_{N} g_{h^{0}}^{f}$ & $g_{N} g_{h^{0}}^{f}$ & $-i g_{N} 2 I_{3}^{f}$ & $i g_{N} 2 I_{3}^{f}$ & $-i \frac{\sin (\beta-\alpha)}{2 s_{w} c_{w}}$ \\
\hline $\mathrm{f}^{\prime} \mathrm{H}^{ \pm} \mathrm{H}^{ \pm}$ & $g_{C}^{\prime} g_{H^{ \pm}}^{f^{\prime}}$ & $g_{C} g_{H^{ \pm}}^{f}$ & $g_{C} g_{H^{ \pm}}^{f}$ & $g_{C}^{\prime} g_{H^{ \pm}}^{f^{\prime}}$ & $-2 I_{3}^{f} \frac{s_{w}^{2}-c_{w}^{2}}{2 s_{w} c_{w}}$ \\
\hline $\mathrm{f}^{\prime} \mathrm{G}^{ \pm} \mathrm{G}^{ \pm}$ & $g_{C}^{\prime} 2 I_{3}^{f^{\prime}}$ & $g_{C} 2 I_{3}^{f}$ & $g_{C} 2 I_{3}^{f}$ & $g_{C}^{\prime} 2 I_{3}^{f^{\prime}}$ & $-2 I_{3}^{f} \frac{s_{w}^{2}-c_{w}^{2}}{2 s_{w} c_{w}}$ \\
\hline $\begin{array}{lll}\tilde{\chi}_{\mathrm{i}}^{0} & \tilde{\mathrm{f}}_{1} & \tilde{\mathrm{f}}_{1} \\
\end{array}$ & $X_{i 1}^{0 f}$ & $\left(Y_{i 1}^{0 f}\right)^{*}$ & $Y_{i 1}^{0 f}$ & $\left(X_{i 1}^{0 f}\right)^{*}$ & $g_{Z, 1}$ \\
\hline $\begin{array}{lll}\tilde{\chi}_{\mathrm{i}}^{0} & \tilde{\mathrm{f}}_{1} & \tilde{\mathrm{f}}_{2} \\
\end{array}$ & $X_{i 2}^{0 f}$ & $\left(Y_{i 2}^{0 f}\right)^{*}$ & $Y_{i 1}^{0 f}$ & $\left(X_{i 1}^{0 f}\right)^{*}$ & $g_{Z}$ \\
\hline $\begin{array}{lll}\tilde{\chi}_{\mathrm{i}}^{0} & \tilde{\mathrm{f}}_{2} & \tilde{\mathrm{f}}_{1} \\
\end{array}$ & $X_{i 1}^{0 f}$ & $\left(Y_{i 1}^{0 f}\right)^{*}$ & $Y_{i 2}^{0 f}$ & $\left(X_{i 2}^{0 f}\right)^{*}$ & $g_{Z}$ \\
\hline $\begin{array}{lll}\tilde{\chi}_{\mathrm{i}}^{0} & \tilde{\mathrm{f}}_{2} & \tilde{\mathrm{f}}_{2} \\
\end{array}$ & $X_{i 2}^{0 f}$ & $\left(Y_{i 2}^{0 f}\right)^{*}$ & $Y_{i 2}^{0 f}$ & $\left(X_{i 2}^{0 f}\right)^{*}$ & $g_{Z, 2}$ \\
\hline$\tilde{\chi}_{\mathrm{i}}^{+} \tilde{\mathrm{f}}_{1}^{\prime} \quad \tilde{\mathrm{f}}_{1}^{\prime}$ & $X_{i 1}^{+f}$ & $\left(Y_{i 1}^{+f}\right)^{*}$ & $Y_{i 1}^{+f}$ & $\left(X_{i 1}^{+f}\right)^{*}$ & $g_{Z, 1}^{\prime}$ \\
\hline $\begin{array}{lll}\tilde{\chi}_{\mathrm{i}}^{+} & \tilde{\mathrm{f}}_{1}^{\prime} & \tilde{\mathrm{f}}_{2}^{\prime} \\
\end{array}$ & $X_{i 2}^{+f}$ & $\left(Y_{i 2}^{+f}\right)^{*}$ & $Y_{i 1}^{+f}$ & $\left(X_{i 1}^{+f}\right)^{*}$ & $g_{Z}^{\prime}$ \\
\hline$\tilde{\chi}_{\mathrm{i}}^{+} \quad \tilde{\mathrm{f}}_{2}^{\prime} \quad \tilde{\mathrm{f}}_{1}^{\prime}$ & $X_{i 1}^{+f}$ & $\left(Y_{i 1}^{+f}\right)^{*}$ & $Y_{i 2}^{+f}$ & $\left(X_{i 2}^{+f}\right)^{*}$ & $g_{Z}^{\prime}$ \\
\hline$\tilde{\chi}_{\mathrm{i}}^{+} \tilde{\mathrm{f}}_{2}^{\prime} \quad \tilde{\mathrm{f}}_{2}^{\prime}$ & $X_{i 2}^{+f}$ & $\left(Y_{i 2}^{+f}\right)^{*}$ & $Y_{i 2}^{+f}$ & $\left(X_{i 2}^{+f}\right)^{*}$ & $g_{Z, 2}^{\prime}$ \\
\hline $\begin{array}{lll}\tilde{g}_{a} & \tilde{\mathrm{q}}_{1} & \tilde{\mathrm{q}}_{1} \\
\end{array}$ & $-\sqrt{2} \frac{g_{3}}{e} c_{\tilde{\theta}} T_{j k}^{a}$ & $\sqrt{2} \frac{g_{3}}{e} s_{\tilde{\theta}} T_{j k}^{a}$ & $\sqrt{2} \frac{g_{3}}{e} s_{\tilde{\theta}} T_{i j}^{a}$ & $-\sqrt{2} \frac{g_{3}}{e} c_{\tilde{\theta}} T_{i j}^{a}$ & $g_{Z, 1}$ \\
\hline $\begin{array}{lll}\tilde{\mathrm{g}}_{\mathrm{a}} & \tilde{\mathrm{q}}_{1} & \tilde{\mathrm{q}}_{2} \\
\end{array}$ & $\sqrt{2} \frac{g_{3}}{e} s_{\tilde{\theta}} T_{j k}^{a}$ & $\sqrt{2} \frac{g_{3}}{e} c_{\tilde{\theta}} T_{j k}^{a}$ & $\sqrt{2} \frac{g_{3}}{e} s_{\tilde{\theta}} T_{i j}^{a}$ & $-\sqrt{2} \frac{g_{3}}{e} c_{\tilde{\theta}} T_{i j}^{a}$ & $g_{Z}$ \\
\hline $\begin{array}{lll}\tilde{g}_{\mathrm{a}} & \tilde{\mathrm{q}}_{2} & \tilde{\mathrm{q}}_{1} \\
\end{array}$ & $-\sqrt{2} \frac{g_{3}}{e} c_{\tilde{\theta}} T_{j k}^{a}$ & $\sqrt{2} \frac{g_{3}}{e} s_{\tilde{\theta}} T_{j k}^{a}$ & $\sqrt{2} \frac{g_{3}}{e} c_{\tilde{\theta}} T_{i j}^{a}$ & $\sqrt{2} \frac{g_{3}}{e} s_{\tilde{\theta}} T_{i j}^{a}$ & $g_{Z}$ \\
\hline $\begin{array}{lll}\tilde{\mathrm{g}}_{\mathrm{a}} & \tilde{\mathrm{q}}_{2} & \tilde{\mathrm{q}}_{2} \\
\end{array}$ & $\sqrt{2} \frac{g_{3}}{e} s_{\tilde{\theta}} T_{j k}^{a}$ & $\sqrt{2} \frac{g_{3}}{e} c_{\tilde{\theta}} T_{j k}^{a}$ & $\sqrt{2} \frac{g_{3}}{e} c_{\tilde{\theta}} T_{i j}^{a}$ & $\sqrt{2} \frac{g_{3}}{e} s_{\tilde{\theta}} T_{i j}^{a}$ & $g_{Z, 2}$ \\
\hline
\end{tabular}




\section{C.5 Class-V diagram}

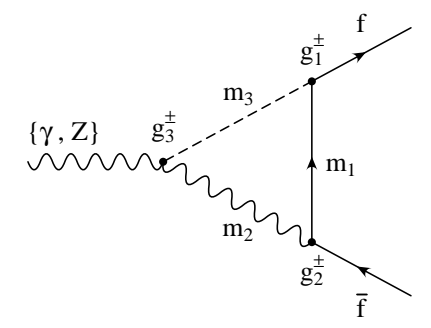

$$
\begin{aligned}
& A_{I I I}^{ \pm}=g_{3}^{ \pm}\left\{\left[m_{f}\left(C_{11}-C_{12}\right) g_{1}^{\mp} g_{2}^{\mp}-C_{12} g_{1}^{ \pm} g_{2}^{ \pm}\right]+m_{1} C_{0} g_{1}^{\mp} g_{2}^{ \pm}\right\} \\
& B_{I I I}^{ \pm}=\left(C_{12}-C_{11}\right) g_{1}^{ \pm} g_{2}^{ \pm} g_{3}^{ \pm} .
\end{aligned}
$$

Masses and coupling constants for the $\gamma f \bar{f}$ vertex $\mathrm{V}$

\begin{tabular}{|c||c|c|c|c|c|}
\hline$m_{1} m_{2} m_{3}$ & $g_{1}^{+}$ & $g_{1}^{-}$ & $g_{2}^{+}$ & $g_{2}^{-}$ & $g_{3}^{+}=g_{3}^{-}$ \\
\hline \hline $\mathrm{f}^{\prime} \quad \mathrm{W} \mathrm{G}^{ \pm}$ & $2 I_{3}^{f^{\prime}} g_{C}^{\prime}$ & $2 I_{3}^{f} g_{C}$ & 0 & $\frac{1}{\sqrt{2} s_{w}}$ & $-M_{W}$ \\
\hline
\end{tabular}

Masses and coupling constants for the $Z f \bar{f}$ vertex $\mathrm{V}$

\begin{tabular}{|c||c|c|c|c|c|}
\hline$m_{1} m_{2} m_{3}$ & $g_{1}^{+}$ & $g_{1}^{-}$ & $g_{2}^{+}$ & $g_{2}^{-}$ & $g_{3}^{+}=g_{3}^{-}$ \\
\hline \hline $\mathrm{f} \quad \mathrm{Z} \mathrm{H} \mathrm{H}^{0}$ & $g_{N} g_{H^{0}}^{f}$ & $g_{N} g_{H^{0}}^{f}$ & $g_{Z, R}$ & $g_{Z, L}$ & $M_{W} \frac{\cos (\beta-\alpha)}{s_{w} c_{w}^{2}}$ \\
\hline $\mathrm{f} \quad \mathrm{Z} \mathrm{h}^{0}$ & $g_{N} g_{h^{0}}^{f}$ & $g_{N} g_{h^{0}}^{f}$ & $g_{Z, R}$ & $g_{Z, L}$ & $M_{W} \frac{\sin (\beta-\alpha)}{s_{w} c_{w}^{2}}$ \\
\hline $\mathrm{f}^{\prime} \quad \mathrm{W} \mathrm{G}^{ \pm}$ & $2 I_{3}^{f^{\prime}} g_{C}^{\prime}$ & $2 I_{3}^{f} g_{C}$ & 0 & $\frac{1}{\sqrt{2} s_{w}}$ & $-M_{W} \frac{s_{w}}{c_{w}}$ \\
\hline
\end{tabular}




\section{C.6 Class-VI diagram}

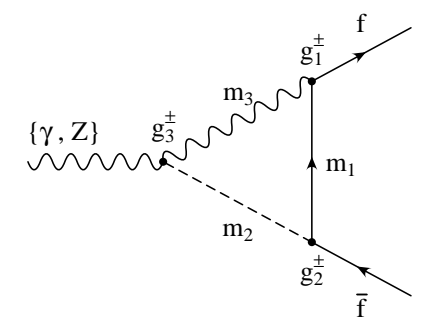

$$
\begin{aligned}
& A_{I V}^{ \pm}=g_{3}^{ \pm}\left\{\left[m_{f}\left(C_{12}-C_{11}\right) g_{1}^{\mp} g_{2}^{ \pm}+C_{12} g_{1}^{ \pm} g_{2}^{\mp}\right]+m_{1} C_{0} g_{1}^{ \pm} g_{2}^{ \pm}\right\} \\
& B_{I V}^{ \pm}=-C_{12} g_{1}^{ \pm} g_{2}^{\mp} g_{3}^{ \pm} .
\end{aligned}
$$

\begin{tabular}{|c||c|c|c|c|c|}
\hline \multicolumn{6}{|c|}{ Masses and coupling constants for the $\gamma f \bar{f}$ vertex VI } \\
\hline$m_{1} m_{2} m_{3}$ & $g_{1}^{+}$ & $g_{1}^{-}$ & $g_{2}^{+}$ & $g_{2}^{-}$ & $g_{3}^{+}=g_{3}^{-}$ \\
\hline \hline $\mathrm{f}^{\prime} \quad \mathrm{G}^{ \pm} \mathrm{W}$ & 0 & $\frac{1}{\sqrt{2} s_{w}}$ & $2 I_{3}^{f} g_{C}$ & $2 I_{3}^{f^{\prime}} g_{C}^{\prime}$ & $-M_{W}$ \\
\hline
\end{tabular}

\begin{tabular}{|c||c|c|c|c|c|}
\hline \multicolumn{6}{|c|}{ Masses and coupling constants for the $Z f \bar{f}$ vertex VI } \\
\hline$m_{1} m_{2} m_{3}$ & $g_{1}^{+}$ & $g_{1}^{-}$ & $g_{2}^{+}$ & $g_{2}^{-}$ & $g_{3}^{+}=g_{3}^{-}$ \\
\hline \hline $\mathrm{f} \quad \mathrm{H}^{0} \mathrm{Z}$ & $g_{Z, R}$ & $g_{Z, L}$ & $g_{N} g_{H^{0}}^{f}$ & $g_{N} g_{H^{0}}^{f}$ & $M_{W} \frac{\cos (\beta-\alpha)}{s_{w} c_{w}^{2}}$ \\
\hline $\mathrm{f} \quad \mathrm{h}^{0} \mathrm{Z}$ & $g_{Z, R}$ & $g_{Z, L}$ & $g_{N} g_{h^{0}}^{f}$ & $g_{N} g_{h^{0}}^{f}$ & $M_{W} \frac{\sin (\beta-\alpha)}{s_{w} c_{w}^{2}}$ \\
\hline $\mathrm{f}^{\prime} \mathrm{G}^{ \pm} \mathrm{W}$ & 0 & $\frac{1}{\sqrt{2} s_{w}}$ & $2 I_{3}^{f} g_{C}$ & $2 I_{3}^{f^{\prime}} g_{C}^{\prime}$ & $-M_{W} \frac{s_{w}}{c_{w}}$ \\
\hline
\end{tabular}




\section{Box diagrams}

Here we list the input for the box diagram contributions in the MSSM, as specified in Appendix A, with exception of the QED graphs involving virtual photons. Because of $m_{e} \ll M_{W}$, Higgs exchange diagrams are negligible.

Masses and couplings of the involved particles are put together in Table D.1 for the direct and in Table D.2 for the crossed vector boson box diagrams, and for the direct and crossed SUSY box diagrams in Tables D.3 to D.6.

Conventions on momenta and internal masses are illustrated in Figure D.1 and in Figure D.2. The arrows within the lines denote the charge flow, and long thin arrows denote the fermion flow if necessary.

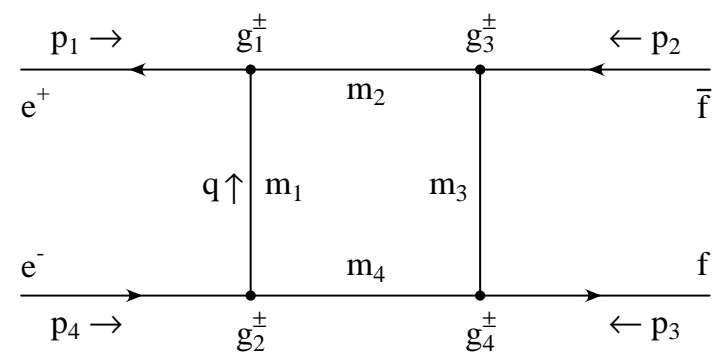

Figure D.1: The topology of the direct box diagrams.

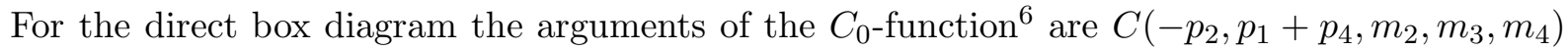
and the arguments of the $D$-functions are $D\left(p_{1}, p_{2}, p_{3}, m_{1}, m_{2}, m_{3}, m_{4}\right)$, with $p_{1}=\bar{p}, p_{2}=-\bar{k}$, $p_{3}=-k$ and $p_{4}=p$.

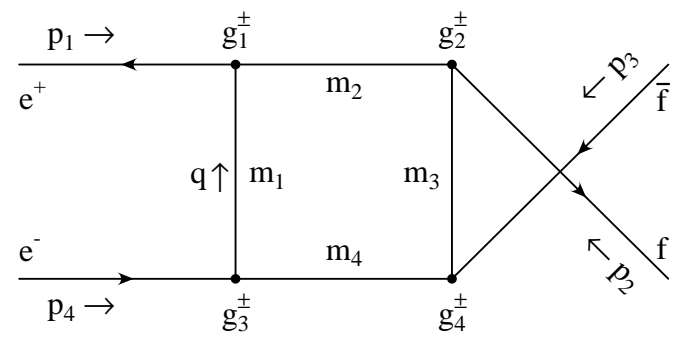

Figure D.2: The topology of the crossed box diagrams.

For the crossed box diagram the arguments of the $C_{0}$-function are $C\left(-p_{2}, p_{1}+p_{4}, m_{2}, m_{3}, m_{4}\right)$ and the arguments of the $D$-functions are $D\left(p_{1}, p_{2}, p_{3}, m_{1}, m_{2}, m_{3}, m_{4}\right)$, with $p_{1}=\bar{p}, p_{2}=-k$, $p_{3}=-\bar{k}$ and $p_{4}=p$.

The couplings $g_{Z, R}$ and $g_{Z, L}$ are defined in Eqs. (C.1), and the SUSY couplings $X_{k l}^{0 f}, X_{k l}^{+f}$, $Y_{k l}^{0 f}$ and $Y_{k l}^{+f}$ in Eqs. (B.15).

\footnotetext{
${ }^{6}$ The arguments of the $C_{0}$-function results here from the reduction of the tensor integral $D_{\mu \nu}$.
} 
D.1 The direct vector boson box diagram

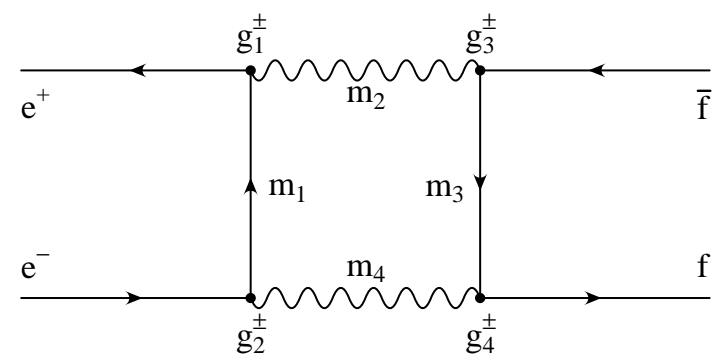

Masses and coupling constants for the direct vector boson box diagram

\begin{tabular}{|c|c|c|c|c|c|c|c|c|}
\hline$m_{1} m_{2} m_{3} m_{4}$ & $g_{1}^{+}$ & $g_{1}^{-}$ & $g_{2}^{+}$ & $g_{2}^{-}$ & $g_{3}^{+}$ & $g_{3}^{-}$ & $g_{4}^{+}$ & $g_{4}^{-}$ \\
\hline e $\mathrm{Z}$ f $\mathrm{Z}$ & $g_{Z, R}^{e}$ & $g_{Z, L}^{e}$ & $g_{Z, R}^{e}$ & $g_{Z, L}^{e}$ & $g_{Z, R}^{f}$ & $g_{Z, L}^{f}$ & $g_{Z, R}^{f}$ & $g_{Z, L}^{f}$ \\
\hline$\nu_{e} \mathrm{~W} \mathrm{f}^{\prime} \mathrm{W}$ & 0 & $\frac{1}{\sqrt{2} s_{w}}$ & 0 & $\frac{1}{\sqrt{2} s_{w}}$ & 0 & $\frac{\frac{1}{2}-I_{3}^{f}}{\sqrt{2} s_{w}}$ & 0 & $\frac{\frac{1}{2}-I_{3}^{f}}{\sqrt{2} s_{w}}$ \\
\hline
\end{tabular}

Table D.1: Entries in the direct vector boson box diagram.

\section{D.2 The crossed vector boson box diagram}

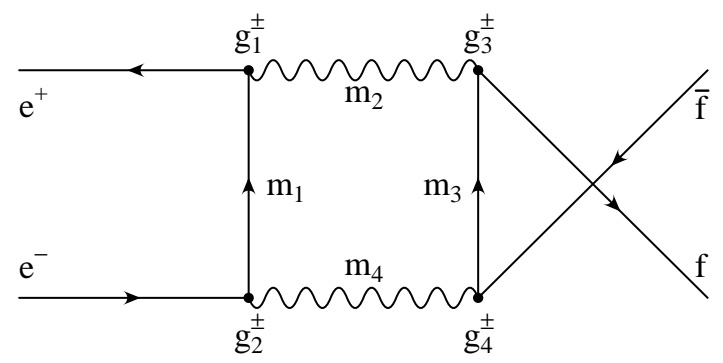

Masses and coupling constants for the crossed vector boson box diagram

\begin{tabular}{|c|c|c|c|c|c|c|c|c|}
\hline$m_{1} m_{2} m_{3} m_{4}$ & $g_{1}^{+}$ & $g_{1}^{-}$ & $g_{2}^{+}$ & $g_{2}^{-}$ & $g_{3}^{+}$ & $g_{3}^{-}$ & $g_{4}^{+}$ & $g_{4}^{-}$ \\
\hline e $Z$ f $Z$ & $g_{Z, R}^{e}$ & $g_{Z, L}^{e}$ & $g_{Z, R}^{e}$ & $g_{Z, L}^{e}$ & $g_{Z, R}^{f}$ & $g_{Z, L}^{f}$ & $g_{Z, R}^{f}$ & $g_{Z, L}^{f}$ \\
\hline$\nu_{e} \mathrm{~W} \quad \mathrm{f}^{\prime} \mathrm{W}$ & 0 & $\frac{1}{\sqrt{2} s_{u}}$ & 0 & $\frac{1}{\sqrt{2} s_{u}}$ & 0 & $\frac{\frac{1}{2}+I_{3}^{f}}{\sqrt{2} s_{w}}$ & 0 & $\frac{\frac{1}{2}+I_{3}^{f}}{\sqrt{2} s_{w}}$ \\
\hline
\end{tabular}

Table D.2: Entries in the crossed vector boson box diagram. 


\section{D.3 The direct neutralino box diagram}

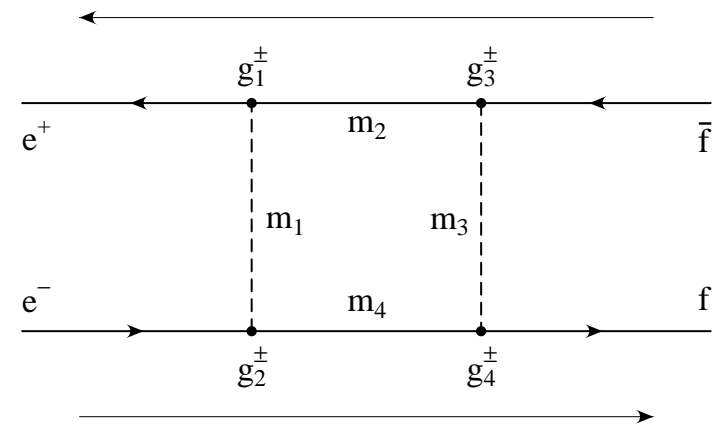

\begin{tabular}{|c|c|c|c|c|c|c|c|c|}
\hline \multicolumn{9}{|c|}{ Masses and coupling constants for the direct neutralino box diagram } \\
\hline$m_{1} m_{2} m_{3} m_{4}$ & $g_{1}^{+}$ & $g_{1}^{-}$ & $g_{2}^{+}$ & $g_{2}^{-}$ & $g_{3}^{+}$ & $g_{3}^{-}$ & $g_{4}^{+}$ & $g_{4}^{-}$ \\
\hline $\begin{array}{lllll}\tilde{e}_{1} & \tilde{\chi}_{j}^{0} & \tilde{f}_{1} & \tilde{\chi}_{i}^{0} \\
\end{array}$ & $X_{j 1}^{0 e}$ & $\left(\bar{C}_{j 1}^{0 e}\right)^{*}$ & $Y_{i 1}^{0 e}$ & $\left(X_{i 1}^{0 e}\right)^{*}$ & $\overline{Y_{j 1}^{0 f}}$ & $\overline{\left(X_{j 1}^{0 f}\right)^{*}}$ & $\overline{X_{i 1}^{0 f}}$ & $\overline{\left(Y_{i 1}^{0 f}\right)^{*}}$ \\
\hline $\begin{array}{llll}\tilde{e}_{1} & \tilde{\chi}_{j}^{0} & \tilde{f}_{2} & \tilde{\chi}_{i}^{0} \\
\end{array}$ & $X_{j 1}^{0 e}$ & $\left(Y_{j 1}^{0 e}\right)^{*}$ & $Y_{i 1}^{0 e}$ & $\left(X_{i 1}^{0 e}\right)^{*}$ & $Y_{j 2}^{0 f}$ & $\left(X_{j 2}^{0 f}\right)^{*}$ & $X_{i 2}^{0 f}$ & $\left(Y_{i 2}^{0 f}\right)^{*}$ \\
\hline $\begin{array}{llll}\tilde{e}_{2} & \tilde{\chi}_{j}^{0} & \tilde{f}_{1} & \tilde{\chi}_{i}^{0} \\
\end{array}$ & $X_{j 2}^{0 e}$ & $\left(Y_{j 2}^{0 e}\right)^{*}$ & $Y_{i 2}^{0 e}$ & $\left(X_{i 2}^{0 e}\right)^{*}$ & $Y_{j 1}^{0 f}$ & $\left(X_{j 1}^{0 f}\right)^{*}$ & $X_{i 1}^{0 f}$ & $\left(Y_{i 1}^{0 f}\right)^{*}$ \\
\hline $\begin{array}{llll}\tilde{e}_{2} & \tilde{\chi}_{j}^{0} & \tilde{f}_{2} & \tilde{\chi}_{i}^{0}\end{array}$ & $X_{j 2}^{0 e}$ & $\left(Y_{j 2}^{0 e}\right)^{*}$ & $Y_{i 2}^{0 e}$ & $\left(X_{i 2}^{0 e}\right)^{*}$ & $Y_{j 2}^{0 f}$ & $\left(X_{j 2}^{0 f}\right)^{*}$ & $\overline{X_{i 2}^{0 f}}$ & $\left(Y_{i 2}^{0 f}\right)^{*}$ \\
\hline
\end{tabular}

Table D.3: Entries in the direct neutralino box diagram.

\section{D.4 The crossed neutralino box diagram}

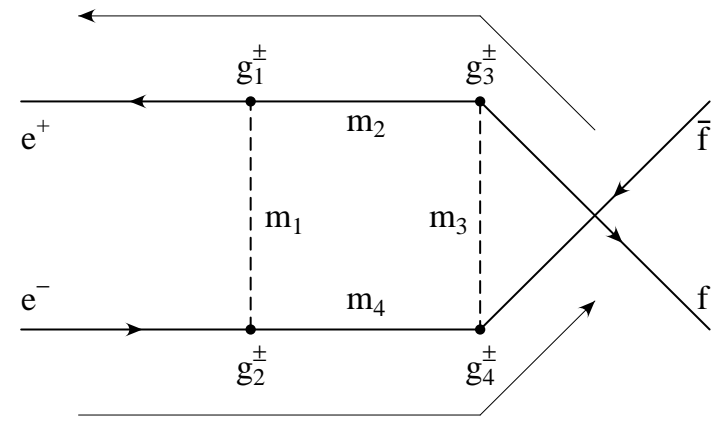

Masses and coupling constants for the crossed neutralino box diagram

\begin{tabular}{|c|c|c|c|c|c|c|c|c|}
\hline$m_{1} m_{2} m_{3} m_{4}$ & $g_{1}^{+}$ & $g_{1}^{-}$ & $g_{2}^{+}$ & $g_{2}^{-}$ & $g_{3}^{+}$ & $g_{3}^{-}$ & $g_{4}^{+}$ & $g_{4}^{-}$ \\
\hline $\begin{array}{llll}\tilde{e}_{1} & \tilde{\chi}_{j}^{0} & \tilde{f}_{1} & \tilde{\chi}_{i}^{0} \\
\end{array}$ & $X_{j 1}^{0 e}$ & 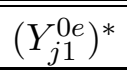 & $Y_{i 1}^{0 e}$ & $\overline{\left(X_{i 1}^{0 e}\right)^{*}}$ & $\overline{X_{j 1}^{0 f}}$ & 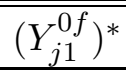 & $Y_{i 1}^{0 f}$ & $\left(X_{i 1}^{0 f}\right)^{*}$ \\
\hline $\begin{array}{llll}\tilde{e}_{1} & \tilde{\chi}_{j}^{0} & \tilde{f}_{2} & \tilde{\chi}_{i}^{0} \\
\end{array}$ & $X_{j 1}^{0 e}$ & $\left(Y_{j 1}^{0 e}\right)^{*}$ & $Y_{i 1}^{0 e}$ & $\left(X_{i 1}^{0 e}\right)^{*}$ & $X_{j 2}^{0 f}$ & $\left(Y_{j 2}^{0 f}\right)^{*}$ & $Y_{i 2}^{0 f}$ & $\left(X_{i 2}^{0 f}\right)^{*}$ \\
\hline $\begin{array}{llll}\tilde{e}_{2} & \tilde{\chi}_{j}^{0} & \tilde{f}_{1} & \tilde{\chi}_{i}^{0} \\
\end{array}$ & $X_{j 2}^{0 e}$ & $\left(Y_{j 2}^{0 e}\right)^{*}$ & $Y_{i 2}^{0 e}$ & $\left(X_{i 2}^{0 e}\right)^{*}$ & $X_{j 1}^{0 f}$ & $\left(Y_{j 1}^{0 f}\right)^{*}$ & $Y_{i 1}^{0 f}$ & $\left(X_{i 1}^{0 f}\right)^{*}$ \\
\hline $\begin{array}{llll}\tilde{e}_{2} & \tilde{\chi}_{j}^{0} & \tilde{f}_{2} & \tilde{\chi}_{i}^{0} \\
\end{array}$ & $X_{j 2}^{0 e}$ & $\left(Y_{j 2}^{0 e}\right)^{*}$ & $Y_{i 2}^{0 e}$ & $\left(X_{i 2}^{0 e}\right)^{*}$ & $X_{j 2}^{0 f}$ & $\left(Y_{j 2}^{0 f}\right)^{*}$ & $Y_{i 2}^{0 f}$ & $\left(X_{i 2}^{0 f}\right)^{*}$ \\
\hline
\end{tabular}

Table D.4: Entries in the crossed neutralino box diagram. 
D.5 The direct chargino box diagram

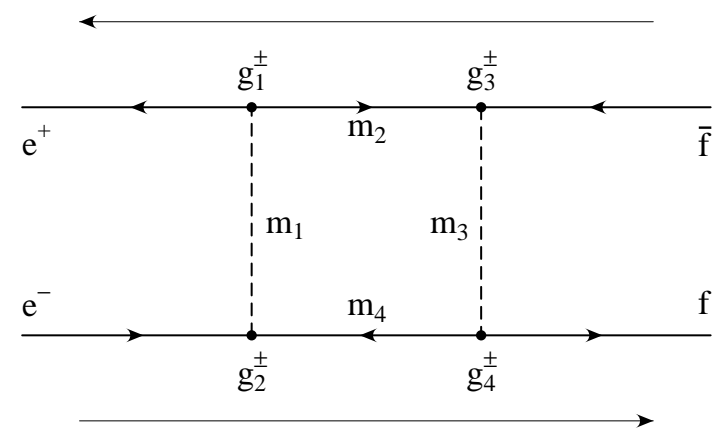

\begin{tabular}{|c|c|c|c|c|c|c|c|c|}
\hline \multicolumn{9}{|c|}{ Masses and coupling constants for the direct chargino box diagram } \\
\hline$m_{1} m_{2} m_{3} m_{4}$ & $g_{1}^{+}$ & $g_{1}^{-}$ & $g_{2}^{+}$ & $g_{2}^{-}$ & $g_{3}^{+}$ & $g_{3}^{-}$ & $g_{4}^{+}$ & $g_{4}^{-}$ \\
\hline 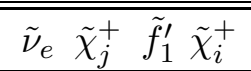 & $X_{j 1}^{+e}$ & $\left(\overline{(Y}_{j 1}^{+e}\right)^{*}$ & $Y_{i 1}^{+e}$ & $\overline{\left(X_{i 1}^{+e}\right)^{*}}$ & $Y_{j 1}^{+f}$ & $\overline{\left(X_{j 1}^{+f}\right)^{*}}$ & $X_{i 1}^{+f}$ & $\left(Y_{i 1}^{+f}\right)^{*}$ \\
\hline $\begin{array}{cccc}\tilde{\nu}_{e} & \tilde{\chi}_{j}^{+} & \tilde{f}_{2}^{\prime} & \tilde{\chi}_{i}^{+} \\
\end{array}$ & $X_{j 1}^{+e}$ & $\left(Y_{j 1}^{+e}\right)^{*}$ & $Y_{i 1}^{+e}$ & $\left(X_{i 1}^{+e}\right)^{*}$ & $Y_{j 2}^{+f}$ & $\left(X_{j 2}^{+f}\right)^{*}$ & $X_{i 2}^{+f}$ & $\left(Y_{i 2}^{+f}\right)^{*}$ \\
\hline
\end{tabular}

Table D.5: Entries in the direct chargino box diagram.

\section{D.6 The crossed chargino box diagram}

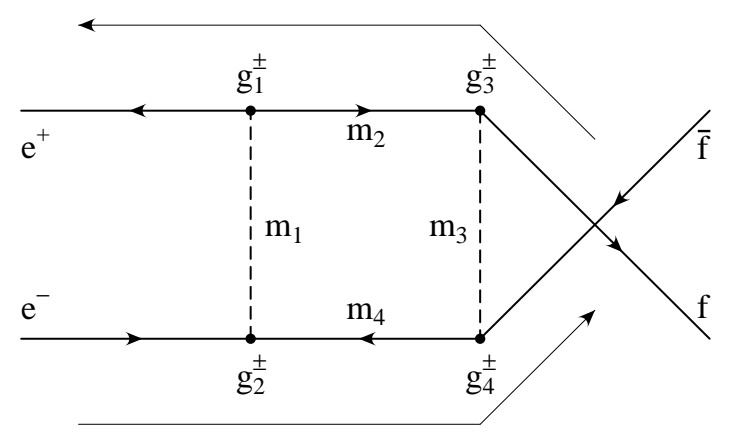

Masses and coupling constants for the crossed chargino box diagram

\begin{tabular}{|c|c|c|c|c|c|c|c|c|}
\hline$m_{1} m_{2} m_{3} m_{4}$ & $g_{1}^{+}$ & $g_{1}^{-}$ & $g_{2}^{+}$ & $g_{2}^{-}$ & $g_{3}^{+}$ & $g_{3}^{-}$ & $g_{4}^{+}$ & $g_{4}^{-}$ \\
\hline $\begin{array}{llll}\tilde{\nu}_{e} & \tilde{\chi}_{j}^{+} & \tilde{f}_{1}^{\prime} & \tilde{\chi}_{i}^{+} \\
\end{array}$ & $\overline{X_{j 1}^{+e}}$ & $\left(Y_{j 1}^{+e}\right)^{*}$ & $Y_{i 1}^{+e}$ & $\left(X_{i 1}^{+e}\right)^{*}$ & $X_{j 1}^{+f}$ & $\left(Y_{j 1}^{+f}\right)^{*}$ & $Y_{i 1}^{+f}$ & $\overline{\left(X_{i 1}^{+f}\right)^{*}}$ \\
\hline$\tilde{\nu}_{e} \tilde{\chi}_{j}^{+} \tilde{f}_{2}^{\prime} \tilde{\chi}_{i}^{+}$ & $X_{j 1}^{+e}$ & $\left(Y_{j 1}^{+e}\right)^{*}$ & $Y_{i 1}^{+e}$ & $\left(X_{i 1}^{+e}\right)^{*}$ & $X_{j 2}^{+f}$ & $\left(Y_{j 2}^{+f}\right)^{*}$ & $Y_{i 2}^{+f}$ & $\left(X_{i 2}^{+f}\right)^{*}$ \\
\hline
\end{tabular}

Table D.6: Entries in the crossed chargino box diagram. 


\section{References}

[1] The LEP Collaborations ALEPH, DELPHI, L3, OPAL, the LEP Electroweak Working Group and the SLD Heavy Flavor Group, A Combination of Preliminary LEP and SLD Electroweak Measurements and Constraints on the Standard Model, CERN-PPE/97-154; D. Karlen, plenary talk at the XXIX Int. Conf. on High Energy Physics, Vancouver, Canada, July 1998.

[2] H.P. Nilles, Phys. Rep. 110 (1984) 1;

M.F. Sohnius, Phys. Rep. 128 (1985) 39.

[3] H.E. Haber, G. Kane, Phys. Rep. 117 (1985) 75.

[4] J.F. Gunion, H.E. Haber, Nucl. Phys. B272 (1986) 1.

[5] J.F. Gunion, H.E. Haber, G. Kane, S. Dawson, The Higgs Hunter's Guide, Frontiers in Physics Vol. 80, Addison-Wesley, 1990.

[6] P. Chankowski, A. Dabelstein, W. Hollik, W. Mösle, S. Pokorski, J. Rosiek, Nucl. Phys. B417 (1994) 101;

D. Garcia, J. Solà, Mod. Phys. Lett. A9 (1994) 211;

D. Garcia, R. Jiménez, J. Solà, Phys. Lett. B347 (1995) 309; Phys. Lett. B347 (1995) 321;

D. Garcia, J. Solà, Phys. Lett. B357 (1995) 349;

A. Dabelstein, W. Hollik, W. Mösle, in Perspectives for Electroweak Interactions in $e^{+} e^{-}$ Collisions, Ringberg Castle 1995, edited by B.A. Kniehl, Word Scientific 1995 (p. 345);

P. Chankowski, S. Pokorski, Nucl. Phys. B475 (1996) 3;

W. de Boer, A. Dabelstein, W. Hollik, W. Mösle, U. Schwickerath, Z. Phys. C75 (1997) 627 ;

J. Bagger, K. Matchev, D. Pierce, R. Zhang, Nucl. Phys. B491 (1997) 3.

[7] A. Djouadi, P. Gambino, S. Heinemeyer, W. Hollik, C. Jünger, G. Weiglein, Phys. Rev. Lett. 78 (1997) 3626; Phys. Rev. D57 (1998) 4179.

[8] C.-H. Chang, C.S. Li, R.J. Oakes, J.M. Yang, Phys. Rev. D51 (1995) 2125.

[9] A. Djouadi, M. Drees, H. König, Phys. Rev. D48 (1993) 3081.

[10] D. Garcia, W. Hollik, R. Jiménez, J. Solà, Nucl. Phys. B427 (1994) 53;

A. Dabelstein, W. Hollik, C. Jünger, R. Jiménez, J. Solà, Nucl. Phys. B454 (1995) 75.

[11] W. Beenakker, S.C. van der Marck, W. Hollik, Nucl. Phys. B365 (1991) 24.

[12] J. Jerzak, E. Laermann, P.M. Zerwas, Phys. Rev. D25 (1980) 1218;

A. Djouadi, Z. Phys. C39 (1988) 561;

K. Chetyrkin, A. Hoang, J. Kühn, M. Steinhauser, T. Teubner, Eur. Phys. J. C2 (1998) 137.

[13] M. Böhm, W. Hollik, H. Spiesberger, Fortschr. Phys. 34 (1986) 687;

W. Hollik, Fortschr. Phys. 38 (1990) 165;

W. Hollik, "Renormalization of the Standard Model" in: Precision Tests of the Standard Model, Advanced series on Directions in High Energy Physics, World Scientific Publishing Co., 1995. 
[14] Reports of the Working Group on Precision Calculations for the Z Resonance, edited by D. Bardin, W. Hollik, G. Passarino, CERN 95-03, Geneva 1995.

[15] G.J. van Oldenborgh, J.A.M Vermaseren, Z. Phys. C46 (1990) 425;

G.J. van Oldenborgh, FF a package to evaluate one-loop Feynman diagrams, 1990, via anonymous ftp from nikhefh.nikhef.nl, NIKHEF-H/90-15.

[16] W. Beenakker, A. Denner, A. Kraft, Nucl. Phys. B410 (1993) 219.

[17] A. Dabelstein, W. Hollik, in: $e^{+} e^{-}$Collisions at 500 GeV: The Physics Potential, Part C, Proceedings of the Workshop, edited by P.M. Zerwas, DESY 93-123C, Hamburg 1993;

A. Dabelstein, Z. Phys. C67 (1995) 495;

A. Dabelstein, Nucl. Phys. B456 (1995) 25.

[18] R.M. Barnett et al., Phys. Rev. D54 (1996) 1 and 1997 off-year partial update for the 1998 edition available on the PDG WWW pages (URL:http://pdg.lbl.gov/).

[19] F. Boudjema, B. Mele et al., "Standard Model Processes" in: Physics at LEP2, edited by G. Altarelli, T. Sjöstrand, F. Zwirner, Vol. 1, CERN 96-01, Geneva 1996.

[20] $e^{+} e^{-}$Collisions at 500 GeV: The Physics Potential, Proceedings of the Workshop, edited by P.M. Zerwas, DESY 92-123A, DESY 92-123B, DESY 93-123C, DESY 96-123D;

P.M. Zerwas et al., Phys. Rep. 299 (1998) 1.

[21] R.J. Guth, J. Kühn, Nucl. Phys. B368 (1992) 38.

[22] W. Beenakker, W. Hollik, Phys. Lett. B269 (1991) 425.

[23] A. Denner, R.J. Guth, J. Kühn, Nucl. Phys. B377 (1992) 3.

[24] T. Appelquist, J. Carazzone, Phys. Rev. D11 (1975) 2856;

A. Dobado, M. Herrero, S. Peñaranda, hep-ph/9710313; hep-ph/9806488.

[25] H. Haber, R. Hempfling, Phys. Rev. Lett. 66 (1991) 1815;

Y. Okada, M. Yamaguchi, T. Yanagida, Prog. Theor. Phys. 85 (1991) 1;

J. Ellis, G. Ridolfi, F. Zwirner, Phys. Lett. B257 (1991) 83; Phys. Lett. B262 (1991) 477;

R. Barbieri, M. Frigeni, Phys. Lett. B258 (1991) 395.

[26] M.A. Díaz, Radiative Corrections to Higgs Masses in the Minimal Supersymmetric Model, Ph.D. Thesis, June 1992, Uni. of California (Santa Cruz), SCIPP-92-13.

[27] G. Passarino, M. Veltman, Nucl. Phys. B160 (1979) 151.

[28] A. Denner, H. Eck, O. Hahn, J. Küblbeck, Nucl. Phys. B387 (1992) 467.

[29] G. 't Hooft, M. Veltman, Nucl. Phys. B153 (1979) 365.

[30] A. Denner, Fortschr. Phys. 41 (1993) 307. 